\title{
Primordial Non-Gaussianities from Inflation Models
}

\author{
Xingang Chen \\ Center for Theoretical Cosmology, Department of Applied Mathematics and Theoretical Physics, University of Cambridge, \\ Cambridge CB3 OWA, UK
}

Correspondence should be addressed to Xingang Chen, x.chen@damtp.cam.ac.uk

Received 15 February 2010; Accepted 9 June 2010

Academic Editor: Sarah Shandera

Copyright $\odot 2010$ Xingang Chen. This is an open access article distributed under the Creative Commons Attribution License, which permits unrestricted use, distribution, and reproduction in any medium, provided the original work is properly cited.

This is a pedagogical review on primordial non-Gaussianities from inflation models. We introduce formalisms and techniques that are used to compute such quantities. We review different mechanisms which can generate observable large non-Gaussianities during inflation, and distinctive signatures they leave on the non-Gaussian profiles. They are potentially powerful probes to the dynamics of inflation. We also provide a nontechnical and qualitative summary of the main results and underlying physics.

\section{Introduction}

An ambitious goal of modern cosmology is to understand the origin of our Universe all the way to its very beginning. To what extent this can be achieved largely depends on what type of observational data we are able to get. Thanks to many modern experiments, we are really making progress in this direction.

One of the representative experiments is the Wilkinson Microwave Anisotropy Probe (WMAP) satellite [1-6]. It measures the light coming from the last scattering surface about 13.7 billions years ago. This cosmic microwave background (CMB) is emitted at about 379,000 years after the Big Bang, when electrons and protons combine to form neutral hydrogen atoms and photons start to travel freely through the space. Our Universe was very young at that moment and the large scale fluctuations were still developing at linear level. So the CMB actually carries valuable information much earlier than itself, which can potentially tell us about the origin of the Big Bang.

There are two amazing facts about the CMB temperature map. On the one hand, it is extremely isotropic, despite the fact that the causally connected region at the time when CMB formed spans an angle of only about 0.8 degree on the sky today. On the other hand, we do observe small fluctuations, with $\Delta T / T \sim 10^{-5}$.

The inflationary scenario [7-9] naturally solves the above two puzzles. It was proposed nearly thirty years ago to address some of the basic problems of the Big Bang cosmology, namely, why the universe is so homogeneous and isotropic. In this scenario, our universe was once dominated by dark energy and had gone through an accelerated expansion phase, during which a Hubble size patch was stretched by more than 60 e-folds or so. Inhomogeneities and large curvature were stretched away by this inflationary epoch, making our current observable universe very homogenous and flat. In the mean while, the fields that were responsible for and participated in this inflationary phase did have quantum fluctuations. These fluctuations also got stretched and imprinted at superhorizon scales. Later they reentered the horizon and seeded the large scale structures today [10-14].

The inflationary scenario has several generic predictions on the properties of the density perturbations that seed the large scale structures.

(i) They are primordial. Namely, they were laid down at superhorizon scales and entering the horizon after the Big Bang.

(ii) They are approximately scale-invariant. This is because, during the 60 e-folds, each mode experiences the similar expansion when they are stretched across the horizon.

(iii) They are approximately Gaussian. In simplest slowroll inflation models, the inflaton is freely propagating in the inflationary background at the leading 
order. This is also found to be true in most of the other models and for different inflationary mechanisms. So the tiny primordial fluctuations can be treated as nearly Gaussian.

The CMB temperature anisotropy is the ideal data that we can use to test these predictions. The obvious first step is to analyze their two-point correlation functions, that is, the power spectrum. All the above predictions are verified to some extent [1]. The presence of the baryon acoustic oscillations proves that the density perturbations are indeed present at the superhorizon scales and reentering the horizon as the horizon expands after the Big Bang. The spectral index, $n_{s}=0.963 \pm 0.012$, is very close to one, therefore, the density perturbations are nearly scale invariant. Several generic types of non-Gaussianities are constrained to be less than one thousandth of the leading Gaussian component.

But is this enough?

Experimentally, the amplitude and the scale-dependence of the power spectrum consist of about 1000 numbers for WMAP. For the Planck satellite, this will be increased up to about 3000. However, we have about one million pixels in the WMAP temperature map alone, and 60 millions for Planck. So the information that we got so far is highly compressed comparing to what the data could offer in principle. This high compression is only justified if the density perturbations are Gaussian within the ultimate sensitivities of our experiments, so all the properties of the map is determined by the two-point function. Otherwise, we are expecting a lot more information in the non-Gaussian components.

Theoretically, inflation still remains as a paradigm. We do not know what kind of fields are responsible for the inflation. We do not know their Lagrangian. We also would like to distinguish inflation from other alternatives. Being our very first data on quantum gravity, we would like to extract the maximum number of information from the CMB map to understand aspects of the quantum gravity. All these motivate us to go beyond the power spectrum.

To give an analogy, in particle physics, two-point correlation functions of fields describe freely propagating particles in Minkowski spacetime. More interesting objects are their higher-order correlations. Measuring these are the goals of particle colliders. Similarly, the power spectrum here describes the freely propagating particles in the inflationary background. To find out more about their interaction details and break the degeneracies among models, we need higherorder correlation functions, namely, non-Gaussianities. So the role non-Gaussianities play for the very early universe is similar to the role colliders play for particle physics.

With these motivations in mind, in this paper, we explore various mechanisms that can generate potentially observable primordial non-Gaussianities, and are consistent with the current results of power spectrum. We will not take the approach of reviewing models one by one. Rather, we divide them into different categories, such that models in each category share the same physical aspect which leaves a unique fingerprint on primordial non-Gaussianities. On the one hand, if any such non-Gaussianity is observed, we know what we have learned concretely in terms of fundamental physics. On the other hand, explicit forms of non-Gaussianities resulted from this exploration provide important clues on how they should be searched in data. Even if the primordial density perturbations were perfectly Gaussian, to test it, we would still go through these analyses until various well-motivated non-Gaussian forms are properly constrained.

1.1. Road Map. The following is the outline of the paper. For readers who would like to get a quick and qualitative understanding of the main results instead of technical details, we also provide a shortcut after the outline.

In Section 2, we review the essential features of the inflation model and density perturbations.

In Section 3, we review the first-principle in-in formalism and related techniques that will be used to calculate the correlation functions in time-dependent background.

In Section 4, we compute the scalar three-point function in the simplest slow-roll model. We list the essential assumptions that lead to the conclusion that the non-Gaussianities in this model is too small to be observed.

In Section 5, we review aspects of inflation model building, emphasizing various generic problems which suggest that the realistic model may not be the algebraically simplest. We also introduce some terminologies used to describe properties of non-Gaussianities.

Sections 6,7 , and 8 contain the main results of this paper. We study various mechanisms that can lead to large nonGaussianities, and their distinctive predictions in terms of the non-Gaussian profile.

In Section 9, we give a qualitative summary of the main results in this paper. Before conclusion, we discuss several useful relations among different non-Gaussianities.

Here is a road map for readers who wish to have a nontechnical explanation and understanding of our main results. After reading the short review on the inflation model and density perturbations in Section 2, one may read the first and the last paragraph of Section 4 to get an idea of the no-go statement, and then directly proceed to read Section 5. After that, one may jump to Section 9 where the main results are summarized in non-technical terms.

The subject of the primordial non-Gaussianities is a fastgrowing one. There exists many nice reviews and books in this and closely related subjects. The introductions to inflation and density perturbations can be found in many textbooks [15-20] and reviews [21-25]. Inflationary model buildings in particle physics, supergravity, and string theory are reviewed in [26-32]. Comprehensive reviews on the developments of theories and observations of primordial non-Gaussianities up to mid 2004 can be found in $[33,34]$. Recent comprehensive reviews on theoretical and observational developments on the bispectrum detection in $\mathrm{CMB}$ and large-scale structure has appeared in $[35,36]$. A recent comprehensive review on non-Gaussianities from the second-order postinflationary evolution of $\mathrm{CMB}$, which acts as contaminations of the primordial non-Gaussianities, has 
appeared in [37]. A recent review on how primordial nonGaussianities can be generated in alternatives to inflation can be found in [38].

\section{Inflation and Density Perturbations}

In this section, we give a quick review on basic elements of inflation and density perturbations. We consider the simplest slow-roll inflation. The action is

$$
\begin{aligned}
S= & \frac{M_{\mathrm{P}}}{2} \int d^{4} x \sqrt{-g} R \\
& +\int d^{4} x \sqrt{-g}\left[-\frac{1}{2} g^{\mu \nu} \partial_{\mu} \phi \partial_{\nu} \phi-V(\phi)\right] .
\end{aligned}
$$

The first term is the Einstein-Hilbert action. The second term describes a canonical scalar field coupled to gravity through the metric $g_{\mu \nu}$. This is the inflaton, which stays on the potential $V(\phi)$ and creates the vacuum energy that drives the inflation. We first study the zero-mode background evolution of the spacetime and the inflaton. The background metric is

$$
d s^{2}=g_{\mu \nu} d x^{\mu} d x^{\nu}=-d t^{2}+a^{2}(t) d \mathbf{x}^{2},
$$

where $a(t)$ is the scale factor and $\mathbf{x}$ is the comoving spatial coordinates. The equations of motion are

$$
\begin{gathered}
H^{2}=\frac{1}{3 M_{\mathrm{P}}^{2}}\left(\frac{1}{2} \dot{\phi}_{0}^{2}+V\right), \\
\dot{H}=-\frac{\dot{\phi}_{0}^{2}}{2 M_{\mathrm{P}}^{2}}, \\
\ddot{\phi}_{0}+3 H \dot{\phi}_{0}+V^{\prime}=0 .
\end{gathered}
$$

The first equation determines the Hubble parameter $H$, which is the expansion rate of the universe. The second equation is the continuity condition. The third equation describes the evolution of the inflaton. Only two of them are independent.

The requirement of having at least $\mathcal{O}(60)$ e-fold of inflation imposes some important conditions. By definition, to have this amount of inflation, the Hubble parameter cannot change much within a Hubble time $H^{-1}$. This gives the first condition

$$
\epsilon \equiv-\frac{\dot{H}}{H^{2}} \ll \mathcal{O}(1)
$$

We also require that the parameter $\epsilon$ does not change much within a Hubble time,

$$
\eta \equiv \frac{\dot{\epsilon}}{\epsilon H} \ll \mathcal{O}(1)
$$

In principle, $\eta$ can be close to $\mathcal{O}(1)$ but $\epsilon$ kept small. In such a case, $\epsilon$ grows exponentially with e-folds and the inflation period tends to be shorter. More importantly, such a case will not generate a scale-invariant spectrum, as we will see shortly, thus cannot be responsible for the CMB. The two conditions (6) and (7) are called the slow-roll conditions. Using the background equations of motion, we can see that the slow-roll conditions impose restrictions on the rolling velocity of the inflaton. The first condition (6) implies that

$$
\frac{\dot{\phi}_{0}^{2}}{2 H^{2} M_{\mathrm{P}}^{2}}=\epsilon \ll \mathcal{O}(1) .
$$

So the energy driving the inflation on the right-hand side of (3) is dominated by the potential. Adding the second condition (7) further implies that

$$
\frac{\ddot{\phi}_{0}}{\dot{\phi}_{0} H}=-\epsilon+\frac{\eta}{2} \ll \mathcal{O}(1) .
$$

So the first term $\ddot{\phi}_{0}$ in (5) is negligible and the evolution of the zero-mode inflaton is determined by the attractor solution

$$
3 H \dot{\phi}_{0}+V^{\prime}=0
$$

Using (10), the slow-roll conditions can also be written in a form that restricts the shape of the potential,

$$
\epsilon_{V} \equiv \frac{M_{\mathrm{P}}^{2}}{2}\left(\frac{V^{\prime}}{V}\right)^{2} \ll \mathcal{O}(1), \quad \eta_{V} \equiv M_{\mathrm{P}}^{2} \frac{V^{\prime \prime}}{V} \ll \mathcal{O}(1) .
$$

They are related to $\epsilon$ and $\eta$ by

$$
\epsilon=\epsilon_{V}, \quad \eta=-2 \eta_{V}+4 \epsilon_{V} .
$$

So the shape of the potential has to be rather flat relative to its height. We emphasize that, although in this example several definitions of the slow-roll conditions are all equivalent, the definitions (6) and (7) are more general. In other cases that we will encounter later in this paper, these two conditions are still necessary to ensure a prolonged inflation and generate a scale-invariant spectrum, but the others no longer have to be satisfied. For example, the shape of potential can be steeper, or the inflationary energy can be dominated by the kinetic energy.

Now let us study the perturbations. To keep things simple but main points illustrated, in this section, we will ignore the perturbations in the gravity sector and only perturb the inflaton,

$$
\phi(\mathbf{x}, t)=\phi_{0}(t)+\delta \phi(\mathbf{x}, t) .
$$

We also ignore terms suppressed by the slow-roll parameters, which we often denote collectively as $\mathcal{O}(\epsilon)$. For example, the mass of the inflaton is $V^{\prime \prime} \sim \mathcal{O}(\epsilon) H^{2}$, and will be ignored. The quadratic Lagrangian for the perturbation theory is simply

$$
L=\int d^{3} x\left[\frac{a^{3}}{2} \dot{\delta} \phi^{2}-\frac{a}{2}\left(\partial_{i} \delta \phi\right)^{2}\right],
$$

and the equation of motion follows:

$$
\ddot{\delta} \phi(\mathbf{k}, t)+3 H \dot{\delta} \phi(\mathbf{k}, t)+\frac{k^{2}}{a^{2}} \delta \phi(\mathbf{k}, t)=0,
$$


where we have written it in the comoving momentum space,

$$
\delta \phi(\mathbf{k}, t)=\int d^{3} x \delta \phi(\mathbf{x}, t) e^{i \mathbf{k} \cdot \mathbf{x}}
$$

The solution to the differential equation (15), $u(\mathbf{k}, t)$, is called the mode function. It is not difficult to check that

$$
a^{3} u(\mathbf{k}, t) \dot{u}^{*}(\mathbf{k}, t)-\text { c.c. }=t \text {-independent const. }
$$

To quantize the perturbations according to the canonical commutation relations between $\delta \phi$ and its conjugate momentum $\delta \pi \equiv \partial L / \partial \delta \dot{\phi}$,

$$
\begin{gathered}
{[\delta \phi(\mathbf{x}, t), \delta \pi(\mathbf{y}, t)]=i \delta(\mathbf{x}-\mathbf{y}),} \\
{[\delta \phi(\mathbf{x}, t), \delta \phi(\mathbf{y}, t)]=0, \quad[\delta \pi(\mathbf{x}, t), \delta \pi(\mathbf{y}, t)]=0,}
\end{gathered}
$$

we decompose

$$
\begin{gathered}
\delta \phi=u(\mathbf{k}, t) a_{\mathbf{k}}+u^{*}(-\mathbf{k}, t) a_{-\mathbf{k}}^{\dagger}, \\
\delta \pi=a^{3} \dot{u}(\mathbf{k}, t) a_{\mathbf{k}}+a^{3} \dot{u}^{*}(-\mathbf{k}, t) a_{-\mathbf{k}}^{\dagger},
\end{gathered}
$$

with the commutation relations

$$
\begin{gathered}
{\left[a_{\mathbf{p}}, a_{-\mathbf{q}}^{\dagger}\right]=(2 \pi)^{3} \delta^{3}(\mathbf{p}+\mathbf{q}),} \\
{\left[a_{\mathbf{p}}, a_{-\mathbf{q}}\right]=0, \quad\left[a_{\mathbf{p}}^{\dagger}, a_{-\mathbf{q}}^{\dagger}\right]=0 .}
\end{gathered}
$$

One can check that the commutation relations (18) and (21) are equivalent because of (17), given that the constant on the right-hand side of (17) is specified to be $i$. This gives the normalization condition for the mode function.

We now write down the mode function explicitly by solving (15):

$$
u(\mathbf{k}, \tau)=C_{+} \frac{H}{\sqrt{2 k^{3}}}(1+i k \tau) e^{-i k \tau}+C_{-} \frac{H}{\sqrt{2 k^{3}}}(1-i k \tau) e^{i k \tau},
$$

where we have used the conformal time $\tau$ defined as $d t \equiv$ $a d \tau$. The infinite past corresponds to $\tau \rightarrow-\infty$ and the infinite future $\tau \rightarrow 0$. We also used the relation $\tau=-1 / \mathrm{Ha}+$ $\mathcal{O}(\epsilon)$. This mode function is a superposition of two linearly independent solutions with the normalization condition

$$
\left|C_{+}\right|^{2}-\left|C_{-}\right|^{2}=1
$$

followed from (17). Consider the limit in which the mode is well within the horizon, that is, its wavelength $a / k$ much shorter than the Hubble length $1 / H$, and consider a time period much shorter than a Hubble time. In these limits, the mode effectively feels the Minkowski spacetime, and the first component in (22) with the positive frequency asymptotes to the vacuum mode of the Minkowski spacetime as we can see from (23). We choose this component as our vacuum choice, and it is usually called the Bunch-Davies state. The annihilation operator $a_{\mathrm{p}}$ annihilates the corresponding Bunch-Davies vacuum, $a_{\mathbf{p}}|0\rangle=0$.
The mode function

$$
u(\mathbf{k}, \tau)=\frac{H}{\sqrt{2 k^{3}}}(1+i k \tau) e^{-i k \tau}
$$

has the following important properties. It is oscillatory within the horizon $k|\tau| \gg 1$. As it gets stretched out of the horizon $k|\tau| \ll 1$, the amplitude becomes a constant and frozen. Physically this means that, if we look at different comoving patches of the universe that have the superhorizon size, and ignore the shorter wavelength fluctuations, they all evolve classically but with different $\delta \phi$. This difference makes them arrive at $\phi_{f}$, the location of the end of inflation, at different times. This space-dependent time difference $\delta t \approx \delta \phi / \dot{\phi}_{0}$ leads to the space-dependent inflationary e-fold difference

$$
\zeta \approx H \delta t \approx H \frac{\delta \phi}{\dot{\phi}_{0}}
$$

Again we ignore terms that are suppressed by the slow-roll parameters. This e-fold difference is the conserved quantity after the mode exits the horizon, and remains so until the mode reenters the horizon sometime after the Big Bang. It is the physical quantity that we can measure, for example, by measuring the temperature anisotropy in the CMB, $\zeta \approx-5 \Delta T / T$ [39]. The information about the primordial inflation is then encoded in the statistical properties of this variable. So we would like to calculate the correlation functions of this quantity. Using (25), (19), (24), and (8), we get the following two-point function:

$$
\left\langle\zeta^{2}\right\rangle \equiv\left\langle 0\left|\zeta\left(\mathbf{k}_{1}, 0\right) \zeta\left(\mathbf{k}_{2}, 0\right)\right| 0\right\rangle=\frac{P_{\zeta}}{2 k_{1}^{3}}(2 \pi)^{5} \delta\left(\mathbf{k}_{1}+\mathbf{k}_{2}\right),
$$

where $P_{\zeta}$ is defined as the power spectrum and in this case it is

$$
P_{\zeta}=\frac{H^{2}}{8 \pi^{2} M_{\mathrm{P}}^{2} \epsilon} .
$$

The spectrum index is defined to be

$$
n_{s}-1 \equiv \frac{d \ln P_{\zeta}}{d \ln k}=-2 \epsilon-\eta,
$$

where the relation $d \ln k=H d t$ is used. If $n_{s}=1$, the spectrum is scale invariant. The current data from CMB tells us that $n_{s}=0.963 \pm 0.012[1]$. So as we have mentioned, this requires a small $\eta$, which is also a value that tends to give more e-folds of inflation.

If this were the end of story, all the primordial density perturbations would be determined by this two-point function and they are Gaussian. The rest of the paper will be devoted to making the above procedure rigorous and to the calculations of higher-order non-Gaussian correlation functions in this and various other models.

\section{In-In Formalism and Correlation Functions}

In this section, we review the in-in formalism and the related techniques that are used to calculate the correlation functions in time-dependent background. The main procedure is summarized in the last subsection. 
3.1. In-In Formalism. We start with the in-in formalism [40-45], following Weinberg's presentation [45].

We are interested in the correlation functions of superhorizon primordial perturbations generated during inflation. So our goal is to calculate the expectation value of an operator $Q$, which is a product in terms of field perturbations $\delta \phi_{a}$ and $\delta \pi_{a}$, at the end of inflation. The subscript $a$ labels different fields. In inflation models, these fields are, for example, the fluctuations of the scalars and metric and their conjugate momenta. In the Heisenberg picture,

$$
\langle Q\rangle \equiv\langle\Omega|Q(t)| \Omega\rangle,
$$

where $t$ is the end of inflation and $|\Omega\rangle$ is the vacuum state for this interacting theory at the far past $t_{0}$.

We start by looking at how the time-dependence in $Q(t)$ is generated.

The Hamiltonian of the system

$$
H[\phi(t), \pi(t)] \equiv \int d^{3} x \mathscr{H}\left[\phi_{a}(\mathbf{x}, t), \pi_{a}(\mathbf{x}, t)\right]
$$

is a functional of the fields $\phi_{a}(\mathbf{x}, t)$ and their conjugate momenta $\pi_{a}(\mathbf{x}, t)$ at a fixed time $t$. On the left-hand side of (30), we have suppressed the variable $\mathbf{x}$ and index $a$ which are integrated or summed over. The $\phi_{a}(\mathbf{x}, t)$ and $\pi_{a}(\mathbf{x}, t)$ satisfy the canonical commutation relations

$$
\begin{gathered}
{\left[\phi_{a}(\mathbf{x}, t), \pi_{b}(\mathbf{y}, t)\right]=i \delta_{a b} \delta^{3}(\mathbf{x}-\mathbf{y}),} \\
{\left[\phi_{a}(\mathbf{x}, t), \phi_{b}(\mathbf{y}, t)\right]=\left[\pi_{a}(\mathbf{x}, t), \pi_{b}(\mathbf{y}, t)\right]=0,}
\end{gathered}
$$

and their evolution is generated by $H$ following the equations of motion:

$$
\begin{aligned}
& \dot{\phi}_{a}(\mathbf{x}, t)=i\left[H[\phi(t), \pi(t)], \phi_{a}(\mathbf{x}, t)\right], \\
& \dot{\pi}_{a}(\mathbf{x}, t)=i\left[H[\phi(t), \pi(t)], \pi_{a}(\mathbf{x}, t)\right] .
\end{aligned}
$$

We consider a time-dependent background, $\bar{\phi}_{a}(\mathbf{x}, t)$ and $\bar{\pi}_{a}(\mathbf{x}, t)$ which are c-numbers and commute with everything, and the perturbations, $\delta \phi_{a}(\mathbf{x}, t)$ and $\delta \pi_{a}(\mathbf{x}, t)$,

$$
\begin{aligned}
& \phi_{a}(\mathbf{x}, t) \equiv \bar{\phi}_{a}(\mathbf{x}, t)+\delta \phi_{a}(\mathbf{x}, t), \\
& \pi_{a}(\mathbf{x}, t) \equiv \bar{\pi}_{a}(\mathbf{x}, t)+\delta \pi_{a}(\mathbf{x}, t) .
\end{aligned}
$$

The background evolution is determined by the classical equations of motion,

$$
\dot{\bar{\phi}}_{a}(\mathbf{x}, t)=\frac{\partial \mathscr{H}}{\partial \bar{\pi}_{a}}, \quad \dot{\bar{\pi}}_{a}(\mathbf{x}, t)=-\frac{\partial \mathscr{H}}{\partial \bar{\phi}_{a}} .
$$

The commutation relations (31) become those for the perturbations,

$$
\begin{gathered}
{\left[\delta \phi_{a}(\mathbf{x}, t), \delta \pi_{b}(\mathbf{y}, t)\right]=i \delta_{a b} \delta^{3}(\mathbf{x}-\mathbf{y})} \\
{\left[\delta \phi_{a}(\mathbf{x}, t), \delta \phi_{b}(\mathbf{y}, t)\right]=\left[\delta \pi_{a}(\mathbf{x}, t), \delta \pi_{b}(\mathbf{y}, t)\right]=0 .}
\end{gathered}
$$

We expand the Hamiltonian as

$$
\begin{aligned}
H[\phi(t), \pi(t)]= & H[\bar{\phi}(t), \bar{\pi}(t)]+\sum_{a} \int d^{3} x \frac{\partial \mathscr{H}}{\partial \bar{\phi}_{a}(\mathbf{x}, t)} \delta \phi_{a}(\mathbf{x}, t) \\
& +\sum_{a} \int d^{3} x \frac{\partial \mathscr{H}}{\partial \bar{\pi}_{a}(\mathbf{x}, t)} \delta \pi_{a}(\mathbf{x}, t) \\
& +\tilde{H}[\delta \phi(t), \delta \pi(t) ; t],
\end{aligned}
$$

where we use $\tilde{H}$ to denote terms of quadratic and higherorders in perturbations. become

Using (34), (35), and (36), the equations of motion (32)

$$
\begin{aligned}
\delta \dot{\phi}_{a}(\mathbf{x}, t) & =i\left[\tilde{H}[\delta \phi(t), \delta \pi(t) ; t], \delta \phi_{a}(\mathbf{x}, t)\right], \\
\delta \dot{\pi}_{a}(\mathbf{x}, t) & =i\left[\tilde{H}[\delta \phi(t), \delta \pi(t) ; t], \delta \pi_{a}(\mathbf{x}, t)\right] .
\end{aligned}
$$

So the evolution of the perturbations, $\delta \phi_{a}$ and $\delta \pi_{a}$, is generated by $\tilde{H}$. It is straightforward to verify that the solutions for (37) are

$$
\begin{aligned}
& \delta \phi_{a}(\mathbf{x}, t)=U^{-1}\left(t, t_{0}\right) \delta \phi_{a}\left(\mathbf{x}, t_{0}\right) U\left(t, t_{0}\right), \\
& \delta \pi_{a}(\mathbf{x}, t)=U^{-1}\left(t, t_{0}\right) \delta \pi_{a}\left(\mathbf{x}, t_{0}\right) U\left(t, t_{0}\right),
\end{aligned}
$$

where $U$ satisfies

$$
\frac{d}{d t} U\left(t, t_{0}\right)=-i \tilde{H}\left[\delta \phi\left(t_{0}\right), \delta \pi\left(t_{0}\right) ; t\right] U\left(t, t_{0}\right)
$$

with the condition at an initial time $t_{0}$ being

$$
U\left(t_{0}, t_{0}\right)=1 \text {. }
$$

To have a systematic scheme to do the perturbation theory, we split $\tilde{H}$ into two parts,

$\tilde{H}[\delta \phi(t), \delta \pi(t) ; t]=H_{0}[\delta \phi(t), \delta \pi(t) ; t]+H_{I}[\delta \phi(t), \delta \pi(t) ; t]$.

The $H_{0}$ is the quadratic kinematic part, which in the perturbation theory will describe the leading evolution of fields. Fields whose evolution are generated by $H_{0}$ are called the interaction picture fields. We add a superscript " $I$ " to label such fields. They satisfy

$$
\begin{aligned}
\delta \dot{\phi}_{a}^{I}(\mathbf{x}, t) & =i\left[H_{0}\left[\delta \phi^{I}(t), \delta \pi^{I}(t) ; t\right], \delta \phi_{a}^{I}(\mathbf{x}, t)\right], \\
\delta \dot{\pi}_{a}^{I}(\mathbf{x}, t) & =i\left[H_{0}\left[\delta \phi^{I}(t), \delta \pi^{I}(t) ; t\right], \delta \pi_{a}^{I}(\mathbf{x}, t)\right] .
\end{aligned}
$$

The solutions are

$$
\begin{aligned}
& \delta \phi_{a}^{I}(\mathbf{x}, t)=U_{0}^{-1}\left(t, t_{0}\right) \delta \phi_{a}\left(\mathbf{x}, t_{0}\right) U_{0}\left(t, t_{0}\right), \\
& \delta \pi_{a}^{I}(\mathbf{x}, t)=U_{0}^{-1}\left(t, t_{0}\right) \delta \pi_{a}\left(\mathbf{x}, t_{0}\right) U_{0}\left(t, t_{0}\right),
\end{aligned}
$$

where $U_{0}$ satisfies

$$
\frac{d}{d t} U_{0}\left(t, t_{0}\right)=-i H_{0}\left[\delta \phi\left(t_{0}\right), \delta \pi\left(t_{0}\right) ; t\right] U_{0}\left(t, t_{0}\right)
$$


with

$$
U_{0}\left(t_{0}, t_{0}\right)=1 .
$$

So the idea is to encode the leading kinematic evolution in terms of the interaction picture fields, and calculate the effects of the interaction through the series expansion in terms of powers of $H_{I}$. To do this, we rewrite (29) as

$$
\begin{aligned}
&\langle\Omega| Q[\left.\delta \phi_{a}(\mathbf{x}, t), \delta \pi_{a}(\mathbf{x}, t)\right]|\Omega\rangle \\
&=\left\langle\Omega\left|U^{-1}\left(t, t_{0}\right) Q\left[\delta \phi_{a}\left(\mathbf{x}, t_{0}\right), \delta \pi_{a}\left(\mathbf{x}, t_{0}\right)\right] U\left(t, t_{0}\right)\right| \Omega\right\rangle \\
&=\langle\Omega| F^{-1}\left(t, t_{0}\right) U_{0}^{-1}\left(t, t_{0}\right) Q\left[\delta \phi_{a}\left(\mathbf{x}, t_{0}\right), \delta \pi_{a}\left(\mathbf{x}, t_{0}\right)\right] \\
& \quad \times U_{0}\left(t, t_{0}\right) F\left(t, t_{0}\right)|\Omega\rangle \\
&=\left\langle\Omega\left|F^{-1}\left(t, t_{0}\right) Q\left[\delta \phi_{a}^{I}(\mathbf{x}, t), \delta \pi_{a}^{I}(\mathbf{x}, t)\right] F\left(t, t_{0}\right)\right| \Omega\right\rangle,
\end{aligned}
$$

where

$$
F\left(t, t_{0}\right) \equiv U_{0}^{-1}\left(t, t_{0}\right) U\left(t, t_{0}\right) .
$$

Using (39), (44), and (41), we get

$$
\begin{aligned}
\frac{d}{d t} F\left(t, t_{0}\right) & =-i U_{0}^{-1}\left(t, t_{0}\right) H_{I}\left[\delta \phi\left(t_{0}\right), \delta \pi\left(t_{0}\right) ; t\right] U_{0}\left(t, t_{0}\right) F\left(t, t_{0}\right) \\
& =-i H_{I}\left[\delta \phi^{I}(t), \delta \pi^{I}(t) ; t\right] F\left(t, t_{0}\right) \\
& \equiv-i H_{I}(t) F\left(t, t_{0}\right),
\end{aligned}
$$

with

$$
F\left(t_{0}, t_{0}\right)=1 .
$$

The solution to (48) and (49) can be written in the following way,

$$
F\left(t, t_{0}\right)=T \exp \left(-i \int_{t_{0}}^{t} H_{I}(t) d t\right)
$$

where the operator $T$ means that, in each term in the Taylor series expansion of the exponential, the time variables have to be time-ordered. The operator $\bar{T}$ will be used to mean the reversed time-ordering.

In summary, the expectation value (29) is

$$
\begin{aligned}
\langle Q\rangle=\left\langle\Omega\left|F^{-1}\left(t, t_{0}\right) Q^{I}(t) F\left(t, t_{0}\right)\right| \Omega\right\rangle, \\
=\langle\Omega|\left[\bar{T} \exp \left(i \int_{t_{0}}^{t} H_{I}(t) d t\right)\right] Q^{I}(t) \\
\times\left[T \exp \left(-i \int_{t_{0}}^{t} H_{I}(t) d t\right)\right]|\Omega\rangle .
\end{aligned}
$$

Notice that in

$$
\begin{gathered}
H_{I}(t) \equiv H_{I}\left[\delta \phi^{I}(t), \delta \pi^{I}(t) ; t\right], \\
Q^{I}(t) \equiv Q\left[\delta \phi_{a}^{I}(\mathbf{x}, t), \delta \pi_{a}^{I}(\mathbf{x}, t)\right],
\end{gathered}
$$

all the field perturbations are in the interaction picture.
The perturbation theory is also often done in terms of the Lagrangian formalism. In the following, we show that they are equivalent. In the above, we perform perturbations on the Hamiltonian, and define $\delta \pi_{a}$ by perturbing $\pi_{a} \equiv \partial L / \partial \dot{\phi}_{a}$, (here we use $\partial$ to denote the functional derivative)

$$
\delta \pi_{a}=\frac{\partial L}{\partial \dot{\phi}_{a}}\left(\phi_{a}, \dot{\phi}_{a}\right)-\frac{\partial L}{\partial \dot{\bar{\phi}}_{a}}\left(\bar{\phi}_{a}, \dot{\bar{\phi}}_{a}\right)
$$

The Hamiltonian $\tilde{H}$ is defined by (36). So using the definition

$$
H \equiv \int d^{3} x \frac{\partial L}{\partial \dot{\phi}_{a}} \dot{\phi}_{a}-L
$$

together with the classical equations of motions (34) and $\dot{\bar{\pi}}_{a}=\partial L / \partial \bar{\phi}_{a}$, the definition (36) for $\tilde{H}$ becomes

$$
\begin{aligned}
\tilde{H}= & \int d^{3} x \frac{\partial L}{\partial \dot{\phi}_{a}}\left(\phi_{a}, \dot{\phi}_{a}\right) \delta \dot{\phi}_{a} \\
& +\int d^{3} x \frac{\partial L}{\partial \bar{\phi}_{a}}\left(\bar{\phi}_{a}, \dot{\bar{\phi}}_{a}\right) \delta \phi_{a}-L\left(\phi_{a}, \dot{\phi}_{a}\right)+L\left(\bar{\phi}_{a}, \dot{\bar{\phi}}_{a}\right) .
\end{aligned}
$$

If we perturb the Lagrangian directly, we keep the part of the Lagrangian that is quadratic and higher in perturbations $\delta \phi_{a}$ and $\delta \dot{\phi}_{a}$,

$$
\begin{aligned}
\widetilde{L}\left(\delta \phi_{a}, \delta \dot{\phi}_{a}, t\right) \equiv & L\left(\phi_{a}, \dot{\phi}_{a}\right)-L\left(\bar{\phi}_{a}, \dot{\bar{\phi}}_{a}\right) \\
& -\int d^{3} x \frac{\partial L}{\partial \bar{\phi}_{a}}\left(\bar{\phi}_{a}, \dot{\bar{\phi}}_{a}\right) \delta \phi_{a} \\
& -\int d^{3} x \frac{\partial L}{\partial \dot{\bar{\phi}}_{a}}\left(\bar{\phi}_{a}, \dot{\bar{\phi}}_{a}\right) \delta \dot{\phi}_{a}
\end{aligned}
$$

The $\delta \pi_{a}$ is defined directly as

$$
\delta \pi_{a} \equiv \frac{\partial \tilde{L}}{\partial\left(\delta \dot{\phi}_{a}\right)}=\frac{\partial L}{\partial \dot{\phi}_{a}}\left(\phi_{a}, \dot{\phi}_{a}\right)-\frac{\partial L}{\partial \dot{\bar{\phi}}_{a}}\left(\bar{\phi}_{a}, \dot{\bar{\phi}}_{a}\right),
$$

where in the second step (56) has been used. So these two definitions of $\delta \pi_{a}$ are equivalent. The Hamiltonian $\tilde{H}$ is defined through $\tilde{L}$,

$$
\tilde{H} \equiv \int d^{3} x \frac{\partial \tilde{L}}{\partial \delta \dot{\phi}_{a}} \delta \dot{\phi}_{a}-\tilde{L} .
$$

Again, using (56), we can see that the two definitions of $\tilde{H}$ are equivalent.

3.2. Mode Functions and Vacuum. The Hamiltonian $H_{0}$ in the above formalism is typically chosen to be the quadratic kinematic terms for field perturbations $\delta \phi_{a}$ without mixing,

$$
H_{0}=\int d^{3} x \sum_{a}\left[\frac{1}{2 A} \delta \pi_{a}^{2}+\frac{B}{2}\left(\partial_{i} \delta \phi_{a}\right)^{2}+\frac{C}{2} \delta \phi_{a}^{2}\right] .
$$


So they describe free fields propagating in the timedependent background. The $A, B$, and $C$ are some timedependent background fields, and they are all positive. The solutions to the equations of motion (42) in momentum space, $u_{a}(\mathbf{k}, t)$, are called the mode functions, where $\mathbf{k}$ denotes the comoving momentum. They satisfy the Wronskian condition

$$
A u_{a}(\mathbf{k}, t) \dot{u}_{a}^{*}(\mathbf{k}, t)-\text { c.c. }=i, \quad(\text { no sum over } a) .
$$

Note that we have specified the time-independent constant on the right-hand side of $(60)$ to be $i$ for the same reason that we see in Section 2. Namely, we decompose $\delta \phi_{a}^{I}$ as

$$
\delta \phi_{a}^{I}(\mathbf{k}, t)=u_{a}(\mathbf{k}, t) a_{a}(\mathbf{k})+u_{a}^{*}(-\mathbf{k}, t) a_{a}^{\dagger}(-\mathbf{k}),
$$

where the annihilation and creation operators satisfy the following relations,

$$
\begin{gathered}
{\left[a_{a}(\mathbf{k}), a_{b}^{\dagger}(-\mathbf{p})\right]=(2 \pi)^{3} \delta_{a b} \delta^{3}(\mathbf{k}+\mathbf{p}),} \\
{\left[a_{a}(\mathbf{k}), a_{b}(-\mathbf{p})\right]=0, \quad\left[a_{a}^{\dagger}(\mathbf{k}), a_{b}^{\dagger}(-\mathbf{p})\right]=0 .}
\end{gathered}
$$

These commutation relations are equivalent to (35) because of (60), but the constant needs to be $i$. This gives the normalization condition for the mode functions.

Being the solutions of the second-order differential equation, generally the mode function is a linear superposition of two independent solutions. So we need to specify the initial condition. For inflation models, as long as the field theory applies, one can always find an early time at which the physical momentum of the mode is much larger than the Hubble parameter and study a time interval much less than a Hubble time. Under these conditions, the equations of motion approach to those in the Minkowski limit, in which the mode function is a linear superposition of two independent plane waves, one with positive frequency and another negative. The ground state in the Minkowski spacetime is the positive one. The mode function which approaches this positive frequency state in the Minkowski limit is called the Bunch-Davies state. In physical coordinates, this limit is proportional to $e^{-i k_{\mathrm{ph}} t}$, (for $k_{\mathrm{ph}} \gg m$ ), where $k_{\mathrm{ph}}$ is the physical momentum. In terms of the conformal time $\tau \equiv \int d t / a(t)$ and the comoving momentum coordinate $k \equiv k_{\mathrm{ph}} / a(t)$ which we often use, this limit is proportional to $e^{-i k \tau}$. We have seen an example in Section 2 and will see more similar examples later with different $A, B$ and $C$. The corresponding vacuum $|0\rangle$ is the Bunch-Davies vacuum and annihilated by $a_{a}(\mathbf{k})$ defined in (61), $a_{a}(\mathbf{k})|0\rangle=0$.

We also would like to write the vacuum of the interacting theory (51) in terms of the vacuum of the free theory $|0\rangle$ defined above. Unlike the scattering theory where the vacuum of the free theory is generally different from the vacuum of the interaction theory, the process that we are studying here do not generate any nontrivial vacuum fluctuations through interactions. This is a direct consequence of the identity

$$
F^{-1} F=1 .
$$

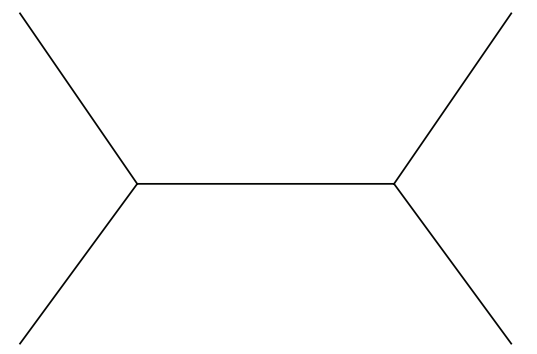

Figure 1: An example of Feynman diagram.

So we can replace $|\Omega\rangle$ in (51) with the Bunch-Davies vacuum $|0\rangle$ that we have specified above.

The integrand $H_{I}(t)$ in (50) is highly oscillatory in the infinite past due to the behavior of the mode function $\propto e^{-i k \tau}$. Their contribution to the integral is averaged out. For the Bunch-Davies vacuum, this regulation can be achieved by introducing a small tilt to the integration contour $\tau_{0} \rightarrow-\infty(1+i \epsilon)$ or performing a Wick rotation $\tau \rightarrow i \tau$. The imaginary component turns the oscillatory behavior into exponentially decay, making the integral well defined.

3.3. Contractions. When evaluating (51), one encounters (anti)time-ordered integrals, of which the integrands are products of fields, such as $\delta \phi_{a}^{I}$ and $\delta \pi_{a}^{I}$, or $\delta \phi_{a}^{I}$ and $\delta \dot{\phi}_{a}^{I}$, sandwiched between the vacua. In contrast to the Minkowski space, in the inflationary background, we do not have a simple analogous Feynman propagator which takes care of the time ordering. Therefore, we will just evaluate the integrands, but leave the complication of the time ordering to the final integration.

To evaluate the integrand, one can shift around the orders of fields in that product, following the rules of the commutation relations. A contraction is defined to be a nonzero commutator between the following components of two fields, $\left[\delta \phi_{a}^{+}, \delta \phi_{b}^{-}\right]$, where $\delta \phi_{a}^{+}$and $\delta \phi_{b}^{-}$denote the first and second term on the right-hand side of (61), respectively. After normal ordering, namely moving annihilation operators to the right-most and creation operators to the left-most so that they give zeros hitting the vacuum, it is not difficult to convince oneself that the only terms left are those with all fields contracted. Feynman diagrams can be used to keep track of what kind of contractions are necessary.

In the following, we demonstrate this using a simple example. We consider a field $\delta \phi^{I}$ and quantize it as usual,

$$
\begin{aligned}
\delta \phi^{I}(\mathbf{k}, t) & \equiv \delta \phi^{+}+\delta \phi^{-} \\
& =u(\mathbf{k}, t) a_{\mathbf{k}}+u^{*}(-\mathbf{k}, t) a_{-\mathbf{k}}^{\dagger}
\end{aligned}
$$

So a contraction between the two terms, $\delta \phi\left(\mathbf{k}, t^{\prime}\right)$ on the left and $\delta \phi\left(\mathbf{p}, t^{\prime \prime}\right)$ on the right, is defined to be 
$\left[\delta \phi^{+}\left(\mathbf{k}, t^{\prime}\right), \delta \phi^{-}\left(\mathbf{p}, t^{\prime \prime}\right)\right]=u\left(\mathbf{k}, t^{\prime}\right) u^{*}\left(-\mathbf{p}, t^{\prime \prime}\right)(2 \pi)^{3} \delta^{3}(\mathbf{k}+\mathbf{p})$.

For example, we want to compute a contribution to the fourpoint function $\left\langle\delta \phi^{4}\right\rangle$ from a tree-diagram containing two three-point interactions of the following form:

$$
H^{I} \propto \int \prod_{i=1}^{3} d \mathbf{p}_{i} \delta \dot{\phi}^{I}\left(\mathbf{p}_{1}, t\right) \delta \dot{\phi}^{I}\left(\mathbf{p}_{2}, t\right) \delta \dot{\phi}^{I}\left(\mathbf{p}_{3}, t\right) .
$$

These two $H^{I}$ s come from expanding $F^{-1}$ or $F$ in (51). The corresponding Feynman diagram is Figure 1.

In Figure 1, the two cubic vertices each represent the three-point interaction (66). Each line represents a contraction. The four outgoing legs connect to the four $\delta \phi\left(\mathbf{p}_{i}, t\right) \quad(i=$ $1,2,3,4)$ in $\left\langle\delta \phi^{4}\right\rangle$. The following is a term from the perturbative series expansion of (51). We demonstrate in the following one set of contractions represented by the diagram in Figure 1,

$$
\begin{aligned}
\delta \dot{\phi}^{I}\left(\mathbf{p}_{1}, t^{\prime}\right) \delta \dot{\phi}^{I}\left(\mathbf{p}_{2}, t^{\prime}\right) \delta \dot{\phi}^{I}\left(\mathbf{p}_{3}, t^{\prime}\right) \delta \phi^{I}\left(\mathbf{k}_{1}, t\right) \delta \phi^{I}\left(\mathbf{k}_{2}, t\right) \delta \phi^{I}\left(\mathbf{k}_{3}, t\right) \delta \phi^{I}\left(\mathbf{k}_{4}, t\right) \delta \dot{\phi}^{I}\left(\mathbf{q}_{1}, t^{\prime \prime}\right) \delta \dot{\phi}^{I}\left(\mathbf{q}_{2}, t^{\prime \prime}\right) \delta \dot{\phi}^{I}\left(\mathbf{q}_{3}, t^{\prime \prime}\right) \\
=\left[\delta \dot{\phi}^{+}\left(\mathbf{p}_{1}, t^{\prime}\right), \delta \phi^{-}\left(\mathbf{k}_{1}, t\right)\right]\left[\delta \dot{\phi}^{+}\left(\mathbf{p}_{2}, t^{\prime}\right), \delta \phi^{-}\left(\mathbf{k}_{2}, t\right)\right]\left[\delta \phi^{+}\left(\mathbf{k}_{3}, t\right), \delta \dot{\phi}^{-}\left(\mathbf{q}_{1}, t^{\prime \prime}\right)\right]\left[\delta \phi^{+}\left(\mathbf{k}_{4}, t\right), \delta \dot{\phi}^{-}\left(\mathbf{q}_{2}, t^{\prime \prime}\right)\right] \\
\quad \times\left[\delta \dot{\phi}^{+}\left(\mathbf{p}_{3}, t^{\prime}\right), \delta \dot{\phi}^{-}\left(\mathbf{q}_{3}, t^{\prime \prime}\right)\right] .
\end{aligned}
$$

Note that all terms are contracted. The result can be further evaluated using (65). After integration over momenta indicated in (66), the final momentum conservation will always manifest itself as $(2 \pi)^{3} \sum_{i}\left(\mathbf{k}_{i}\right)$. There are other sets of contractions represented by the same diagram for the same term. Namely, there are three ways of picking two of the three $\mathbf{p}_{i} s\left(\mathbf{q}_{i} s\right)$, so we have a symmetry factor 9 ; also, there are 24 permutations of the four $\mathbf{k}_{i}$ s. We need to sum over all these possibilities. We also need to sum over all possible terms containing two $H^{I}$ s in the perturbative series, which are not listed here, with their corresponding time ordered integral structure.

3.4. Three Forms. Now we deal with the time ordered integrals in the series expansion. There are two ways to expand (51).

In the first form, we simply expand the exponential in (50). For example, for an even $n$, the $n$th order term is

$$
\begin{aligned}
i^{n}( & -1)^{n / 2} \int_{t_{0}}^{t} d \bar{t}_{1} \int_{t_{0}}^{\bar{t}_{1}} d \bar{t}_{2} \cdots \int_{t_{0}}^{\bar{t}_{n / 2-1}} d \bar{t}_{n / 2} \\
& \times \int_{t_{0}}^{t} d t_{1} \int_{t_{0}}^{t_{1}} d t_{2} \cdots \int_{t_{0}}^{t_{n / 2-1}} d t_{n / 2} \\
& \times\left\langle H_{I}\left(\bar{t}_{n / 2}\right) \cdots H_{I}\left(\bar{t}_{1}\right) Q_{I}(t) H_{I}\left(t_{1}\right) \cdots H_{I}\left(t_{n / 2}\right)\right\rangle \\
& +2 \operatorname{Re} \sum_{m=1}^{n / 2} i^{n}(-1)^{m+n / 2} \int_{t_{0}}^{t} d \bar{t}_{1} \int_{t_{0}}^{\bar{t}_{1}} d \bar{t}_{2} \cdots \int_{t_{0}}^{\bar{t}_{n / 2-1-m}} d \bar{t}_{n / 2-m} \\
& \times \int_{t_{0}}^{t} d t_{1} \int_{t_{0}}^{t_{1}} d t_{2} \cdots \int_{t_{0}}^{t_{n / 2-1+m}} d t_{n / 2+m} \\
& \times\left\langle H_{I}\left(\bar{t}_{n / 2-m}\right) \cdots H_{I}\left(\bar{t}_{1}\right) Q_{I}(t) H_{I}\left(t_{1}\right) \cdots H_{I}\left(t_{n / 2+m}\right)\right\rangle .
\end{aligned}
$$

Each term in the above summation contains two factors of multiple integrals, one from $F^{-1}$ and another from $F$. Each multiple integral is time ordered or antitime-ordered, but there is no time ordering between the two. We call this representation the factorized form.
In the second form, we rearrange the factorized form so that all the time variables are time-ordered, and all the integrands are under a unique integral. The $n$th order term in this form is [45]

$$
\begin{aligned}
& i^{n} \int_{t_{0}}^{t} d t_{1} \int_{t_{0}}^{t_{1}} d t_{2} \cdots \int_{t_{0}}^{t_{n-1}} d t_{n} \\
& \quad \times\left\langle\left[H_{I}\left(t_{n}\right),\left[H_{I}\left(t_{n-1}\right), \ldots,\left[H_{I}\left(t_{1}\right), Q_{I}(t)\right] \cdots\right]\right]\right\rangle .
\end{aligned}
$$

We call this representation the commutator form.

Each representation has its computational advantages and disadvantages.

The factorized form is most convenient to achieve the UV $\left(t_{i} \rightarrow t_{0}\right)$ convergence. As mentioned, after we tilt or rotate the integration contour into the positive imaginary plane for the left integral, and negative imaginary plane for the right integral, all the oscillatory behavior in the UV becomes well-behaved exponential decay. However, this form is not always convenient to deal with the IR $\left(t_{i} \rightarrow\right.$ $t$ ) behavior. For cases where the correlation functions have some nontrivial evolution after modes exit the horizon, as typically happens for inflation models with multiple fields, the convergence in the IR is slow. Cancellation of spurious leading contributions from different terms in the sum (68) can be very implicit in this representation, and could easily lead to wrong leading order results in analytical estimation or numerical evaluation.

The commutator form is most convenient to get the correct leading order behavior in the IR. The mutual cancellation between different terms are made explicit in terms of the nested commutators, before the multiple integral is performed. However, such a regrouping of integrands makes the UV convergence very implicit. Recall that the contour deformation is made to damp the oscillatory behavior in the infinite past. In the commutator form, for any individual term in the integrand, we can still generically choose a unique convergence direction in terms of contour deformation. Although the directions are different for different terms, they can be separately chosen for each of them. But now the problem is, if these different terms have to be grouped as in the 
nested commutator so that the IR cancellation is explicit, the two directions get mixed. Hence, the explicit IR cancellation is incompatible with the explicit UV convergence in this case.

To take advantage of both forms, we introduce a cutoff $t_{c}$ and write the IR part of the in-in formalism in terms of the commutator form and the UV part in terms of the factorized form [46],

$$
\begin{aligned}
& \sum_{i=1}^{n} \int_{t_{c}}^{t} d t_{1} \cdots \int_{t_{c}}^{t_{i-1}} d t_{i}\{\text { commutator form }\} \\
& \quad \times \int_{-\infty}^{t_{c}} d t_{i+1} \cdots \int_{-\infty}^{t_{n-1}} d t_{n}\{\text { factorized form }\} .
\end{aligned}
$$

This representation is called the mixed form. This form is particularly efficient in numerical computations when combined with the Wick-rotations in the UV.

We will not always encounter all these subtleties in every model, but there does exist such interesting examples, as we will see in Section 7.1.

3.5. Summary. To end this section, we summarize the procedure that we need to go through to calculate the correlation functions in the in-in formalism.

Starting with the Lagrangian $L[\phi(t), \dot{\phi}(t)]$, we perturb it around the homogenous solutions $\bar{\phi}_{a}$ and $\dot{\bar{\phi}}_{a}$ of the classical equations of motion,

$$
\begin{aligned}
& \phi_{a}(\mathbf{x}, t)=\bar{\phi}_{a}(t)+\delta \phi_{a}(\mathbf{x}, t), \\
& \dot{\phi}_{a}(\mathbf{x}, t)=\dot{\bar{\phi}}_{a}(t)+\delta \dot{\phi}_{a}(\mathbf{x}, t) .
\end{aligned}
$$

Keep the part of the Lagrangian that is quadratic and higher in perturbations and denote it as $\widetilde{L}$. Define the conjugate momentum densities as $\delta \pi_{a}=\partial \tilde{L} / \partial\left(\delta \dot{\phi}_{a}\right)$. We can also equivalently expand the Hamiltonian $H[\phi(t), \pi(t)]$ by perturbing $\phi_{a}(\mathbf{x}, t)$ and $\pi_{a}(\mathbf{x}, t)$.

Work out the Hamiltonian in terms of $\delta \phi_{a}$ and $\delta \pi_{a}$, and separate them into the quadratic kinematic part $H_{0}$, which describes the free fields in the time-dependent background, and the interaction part $H_{I}$. Relabel $\delta \phi_{a} s$ and $\delta \pi_{a} s$ in the Hamiltonian density as the interaction picture fields, $\delta \phi_{a}^{I} \mathrm{~s}$ and $\delta \pi_{a}^{I}$ s. These latter variables satisfy the equations of motion followed from the $H_{0}$. We quantize $\delta \phi_{a}^{I}$ and $\delta \pi_{a}^{I}$ in terms of the annihilation and creation operators as in (61) and (62). The mode functions $u_{a}(\mathbf{k}, t)$ are solutions of the equations of motion from $H_{0}$, normalized according to the Wronskian conditions (60) and specified by an initial condition such as the Bunch-Davies vacuum. The correlation function for $Q(t)$ is given by

$$
\begin{aligned}
\langle Q(t)\rangle \equiv\langle 0| & {\left[\bar{T} \exp \left(i \int_{t_{0}}^{t} H_{I}(t) d t\right)\right] Q^{I}(t) } \\
& \times\left[T \exp \left(-i \int_{t_{0}}^{t} H_{I}(t) d t\right)\right]|0\rangle,
\end{aligned}
$$

where $Q(t)$ is a product in terms of $\delta \phi_{a}^{I}(\mathbf{x}, t)$ and $\delta \pi^{I}(\mathbf{x}, t)$. If we want to work with $\delta \phi_{a}^{I}$ and $\delta \dot{\phi}_{a}^{I}$ instead of $\delta \phi_{a}^{I}$ and $\delta \pi_{a}^{I}$, we replace $\delta \pi_{a}^{I}$ with $\delta \dot{\phi}_{a}^{I}$ using the relation $\delta \dot{\phi}_{a}^{I}=\partial H_{0} / \partial\left(\delta \pi_{a}^{I}\right)$.
Choose appropriate forms in Section 3.4 and seriesexpand the integrand in powers of $H_{I}$ to the desired orders. Perform contractions defined in Section 3.3 for each term in this expansion. Each term gives a nonzero contribution only when all fields are contracted. Draw Feynman diagrams that represent the correlation functions, and use them as a guidance to do contractions. Finally sum over all possible contractions and perform the time-ordered integrations.

\section{A No-Go Theorem}

Simplest inflation models generate negligible amount of non-Gaussianities that are well below our current experimental abilities $[47,48]$. By simplest, we mean

(i) single scalar field inflation

(ii) with canonical kinetic term

(iii) always slow-rolls

(iv) in Bunch-Davies vacuum

(v) in Einstein gravity.

This list is extracted based on Maldacena's computation of three-point functions in an explicit slow-roll model [47]. We now review this proof. The notations here follow $[49,50]$ and will be consistently used later in this paper.

The Lagrangian for the single scalar field inflation with canonical kinetic term is the following:

$$
S=\int d^{4} x \sqrt{-g}\left[\frac{M_{\mathrm{P}}}{2} R+X-V(\phi)\right],
$$

where $\phi$ is the inflaton field, $X=-(1 / 2) g^{\mu \nu} \partial_{\mu} \phi \partial_{\nu} \phi$ is the canonical kinetic term and $V$ is the slow-roll potential. The first term is the Einstein gravity and $M_{\mathrm{P}}=(8 \pi G)^{-1 / 2}$ is the reduced Planck mass. For convenience we will set the reduced Planck mass $M_{\mathrm{P}}=1$. The signature of the metric is $(-1,1,1,1)$.

The inflaton starts near the top of the potential and slowly rolls down. As we have reviewed in Section 2, to ensure that the inflation lasts for at least $\mathcal{O}(60) \mathrm{e}$-folds, the potential is required to be flat so that the slow-roll parameters (11) are both much less than one most of the time. The energy of the universe is dominated by the potential energy, and the inflaton follows the slow-roll attractor solution (10). Also as discussed in Section 2, we will use the following more general slow-roll parameters:

$$
\epsilon=-\frac{\dot{H}}{H^{2}}, \quad \eta=\frac{\dot{\epsilon}}{\epsilon H} .
$$

To study the perturbation theory, it is convenient to use the ADM formalism, in which the metric takes the form

$$
d s^{2}=-N^{2} d t^{2}+h_{i j}\left(d x^{i}+N^{i} d t\right)\left(d x^{j}+N^{j} d t\right) .
$$

The action becomes

$$
\begin{aligned}
S= & \frac{1}{2} \int d t d x^{3} \sqrt{h} N\left(R^{(3)}+2 X-2 V\right) \\
& +\frac{1}{2} \int d t d x^{3} \sqrt{h} N^{-1}\left(E_{i j} E^{i j}-E^{2}\right),
\end{aligned}
$$


where the index of $N^{i}$ can be lowered by the 3D metric $h_{i j}$ and $R^{(3)}$ is the $3 \mathrm{D}$ Ricci scalar constructed from $h_{i j}$. The definitions of $E_{i j}$ and $E$ are

$$
\begin{gathered}
E_{i j}=\frac{1}{2}\left(\dot{h}_{i j}-\nabla_{i} N_{j}-\nabla_{j} N_{i}\right), \\
E=E_{i j} h^{i j} .
\end{gathered}
$$

In the ADM formalism, the variables $N$ and $N^{i}$ are Lagrangian multipliers whose equations of motion are easy to solve. In single field inflation, we have only one physical scalar perturbation [51]. We choose the uniform inflaton gauge (also called the comoving gauge) in which the scalar perturbation $\zeta$ appears in the three dimensional metric $h_{i j}$ in the following form:

$$
h_{i j}=a^{2} e^{2 \zeta} \delta_{i j}
$$

and the inflaton fluctuation $\delta \phi$ vanishes. The $a(t)$ is the homogeneous scale factor of the universe, so $\zeta$ is a spacedependent rescaling factor. In this paper we do not consider the tensor perturbations.

We plug (75) and (78) into the action (76) and solve the constraint equations for the Lagrangian multipliers $N$ and $N^{i}$. We then plug them back to the action and expand up to the cubic order in $\zeta$ in order to calculate the three-point functions. To do this, in the ADM formalism, it is enough to solve $N$ and $N^{i}$ to the first-order in $\zeta$. This is because their third-order perturbations will multiply the zeroth order constraint equation which vanishes, and their secondorder perturbations will multiply the first-order constraint equation which again vanishes. After some lengthy algebra, we obtain the following expansions:

$$
\begin{gathered}
S_{2}=\int d t d^{3} x\left[a^{3} \epsilon \dot{\zeta}^{2}-a \epsilon(\partial \zeta)^{2}\right] \\
S_{3}=\int d t d^{3} x\left[a^{3} \epsilon^{2} \zeta \dot{\zeta}^{2}+a \epsilon^{2} \zeta(\partial \zeta)^{2}-2 a \epsilon \dot{\zeta}(\partial \zeta)(\partial \chi)\right. \\
+\frac{a^{3} \epsilon}{2} \dot{\eta} \zeta^{2} \dot{\zeta}+\frac{\epsilon}{2 a}(\partial \zeta)(\partial \chi) \partial^{2} \chi \\
\left.+\frac{\epsilon}{4 a}\left(\partial^{2} \zeta\right)(\partial \chi)^{2}+\left.f(\zeta) \frac{\delta L}{\delta \zeta}\right|_{1}\right]
\end{gathered}
$$

where

$$
\begin{gathered}
\chi=a^{2} \epsilon \partial^{-2} \dot{\zeta}, \\
\left.\frac{\delta L}{\delta \zeta}\right|_{1}=2 a\left(\frac{d \partial^{2} \chi}{d t}+H \partial^{2} \chi-\epsilon \partial^{2} \zeta\right), \\
f(\zeta)=\frac{\eta}{4} \zeta^{2}+\text { terms with derivatives on } \zeta
\end{gathered}
$$

Here $\partial^{-2}$ is the inverse Laplacian and $\delta L /\left.\delta \zeta\right|_{1}$ is the variation of the quadratic action with respect to the perturbation $\zeta$. We now can follow Section 3 and proceed to calculate the correlation functions. For simplicity, we will always neglect the superscript " $I$ " on various interaction picture fields.
We restrict to the case where the slow-roll parameters are always small and featureless. We first look at the quadratic action. In this case, we can analytically solve the equation of motion followed from (79) in terms of the Fourier mode of $\zeta$,

$$
u_{k}=\int d^{3} x \zeta(t, \mathbf{x}) e^{-i \mathbf{k} \cdot \mathbf{x}}
$$

and get the mode function

$$
u_{k}=u(\mathbf{k}, \tau)=\frac{i H}{\sqrt{4 \epsilon k^{3}}}(1+i k \tau) e^{-i k \tau},
$$

where $\tau \equiv \int d t / a \approx-(a H)^{-1}$ is the conformal time. The normalization is determined by the Wronskian condition (60). We have chosen the Bunch-Davies vacuum by imposing the condition that the mode function approaches the vacuum state of the Minkowski spacetime in the short wavelength limit $k / a \gg 1 / H$,

$$
u_{k} \longrightarrow-\frac{H \tau}{\sqrt{4 \epsilon k}} e^{-i k \tau}
$$

The dynamical behavior of $\zeta$ that has been explained around (24) and (25) is made precise here. In particular, $\zeta$ is exactly massless without dropping any $\mathcal{O}(\epsilon)$ suppressed terms. In addition, from (78), we can see that, for superhorizon modes, the only effect of $\zeta$ is to provide a homogeneous spatial rescaling. And $\zeta$ is the only scalar perturbation. So the fact that $\zeta$ is frozen after horizon exit will not be changed by higher-order terms.

If we choose the spatially flat gauge, we make $\zeta$ disappear and the scalar in this perturbation theory becomes the perturbation of $\phi$. The relation between $\zeta$ and $\delta \phi$ in (25) (with $\mathcal{O}(\epsilon)$ corrections) is thus a gauge transformation through a space-dependent time shift.

We quantize the field as

$$
\zeta(\mathbf{k}, \tau)=u_{k} a_{\mathbf{k}}+u_{k}^{*} a_{-\mathbf{k}}^{\dagger}
$$

with the canonical commutation relation $\left[a_{\mathbf{k}}, a_{\mathbf{k}^{\prime}}^{\dagger}\right]=$ $(2 \pi)^{3} \delta^{3}\left(\mathbf{k}-\mathbf{k}^{\prime}\right)$. We can easily compute the two-point function at the tree level,

$$
\left\langle\zeta\left(\mathbf{k}_{1}\right) \zeta\left(\mathbf{k}_{2}\right)\right\rangle=\frac{P_{\zeta}}{2 k_{1}^{3}}(2 \pi)^{5} \delta^{3}\left(\mathbf{k}_{1}+\mathbf{k}_{2}\right),
$$

where

$$
P_{\zeta}=\frac{H^{2}}{8 \pi^{2} \epsilon}
$$

Since $\zeta$ remains constant after it exits the horizon, the $H$ and $\epsilon$ are both evaluated near the horizon exit.

We next look at the cubic action. For single field models, $H_{I, 3}=-L_{3}$. Keeping in mind that $\chi$ is proportional to $\epsilon$, one can see that the first line of $(80)$ is proportional to $\epsilon^{2}$. For the featureless potential, $\dot{\eta}=\mathcal{O}\left(\epsilon^{2}\right)$, where $\epsilon$ collectively denotes either $\epsilon$ or $\eta$. So the second line of (80) is proportional to $\epsilon^{3}$, and negligible. The third line can be absorbed by a field redefinition $\zeta \rightarrow \zeta_{n}+f\left(\zeta_{n}\right)$. The only term in $f\left(\zeta_{n}\right)$ 
that will contribute to the correlation function is written out explicitly in (83). All the others involve derivatives of $\zeta$ so vanish outside the horizon. Thus this redefinition eventually introduces an extra term,

$$
\begin{aligned}
& \left\langle\zeta\left(\mathbf{k}_{1}\right) \zeta\left(\mathbf{k}_{2}\right) \zeta\left(\mathbf{k}_{3}\right)\right\rangle \\
& =\left\langle\zeta_{n}\left(\mathbf{k}_{1}\right) \zeta_{n}\left(\mathbf{k}_{2}\right) \zeta_{n}\left(\mathbf{k}_{3}\right)\right\rangle \\
& \quad+\frac{\eta}{4}\left(\left\langle\zeta_{n}^{2}\left(\mathbf{k}_{1}\right) \zeta_{n}\left(\mathbf{k}_{2}\right) \zeta_{n}\left(\mathbf{k}_{3}\right)\right\rangle+2 \text { perm. }\right)+\mathcal{O}\left(\eta^{2}\left(P_{k}^{\zeta}\right)^{3}\right)
\end{aligned}
$$

According to (72), we expand the exponential to the firstorder in $H_{I, 3}$ to get the leading result

$$
\left\langle\zeta_{n}^{3}\right\rangle=-i\left\langle 0|| \int_{t_{0}}^{t} d t\left[\zeta_{n}\left(\mathbf{k}_{1}\right) \zeta_{n}\left(\mathbf{k}_{2}\right) \zeta_{n}\left(\mathbf{k}_{3}\right), H_{I, 3}\right]|| 0\right\rangle .
$$

To estimate the order of magnitude of the bispectrum, we only need to keep track of the factors of $H$ and $\epsilon$. For example, from the first term in (80), we have $\int d t H_{3}(t) \supset$ $-\int d x^{3} d \tau a^{2} \epsilon^{2} \zeta \zeta^{\prime 2}$, where we used the conformal time $\tau$ and the prime denotes the derivative to $\tau$. Using $a \propto H^{-1}, \zeta \propto$ $\zeta^{\prime} \propto H / \sqrt{\epsilon}$, we see that this three-point vertex contributes $\propto H \sqrt{\epsilon}$. Together with the three external legs $\zeta^{3}$ and the definition $P_{\zeta} \propto H^{2} / \epsilon$, we get

$$
\left\langle\zeta^{3}\right\rangle=-i \int d t\left\langle\left[\zeta^{3}, H_{I, 3}(t)\right]\right\rangle \propto \frac{H^{4}}{\epsilon} \propto \mathcal{O}(\epsilon) P_{\zeta}^{2}
$$

Similar results can be obtained for the other two terms in the first line of (80). As we will define more carefully later, the size of the three-point function is conventionally characterized by the number $f_{\mathrm{NL}}$, which is defined as $\left\langle\zeta^{3}\right\rangle \sim$ $f_{\mathrm{NL}} P_{\zeta}^{2}$. So the contribution from the first line of $(80)$ is $f_{\mathrm{NL}}=\mathcal{O}(\epsilon)$. The extra term due to the redefinition (90) contributes $f_{\mathrm{NL}}=\mathcal{O}(\eta)$. This completes the order-ofmagnitude estimate. To get the full non-Gaussian profile, we need to compute the integrals explicitly and get

$$
\left\langle\zeta\left(\mathbf{k}_{1}\right) \zeta\left(\mathbf{k}_{2}\right) \zeta\left(\mathbf{k}_{3}\right)\right\rangle=(2 \pi)^{7} \delta^{3}\left(\mathbf{k}_{1}+\mathbf{k}_{2}+\mathbf{k}_{3}\right) P_{\zeta}^{2} \frac{1}{\prod_{i} k_{i}^{2}} S,
$$

where

$$
\begin{aligned}
S=\frac{\epsilon}{8}[ & -\left(\frac{k_{1}^{2}}{k_{2} k_{3}}+2 \text { perm. }\right)+\left(\frac{k_{1}}{k_{2}}+5 \text { perm. }\right) \\
& \left.+\frac{8}{K}\left(\frac{k_{1} k_{2}}{k_{3}}+2 \text { perm. }\right)\right]+\frac{\eta}{8}\left(\frac{k_{1}^{2}}{k_{2} k_{3}}+2 \text { perm. }\right),
\end{aligned}
$$

where $K=k_{1}+k_{2}+k_{3}$ and the permutations stand for those among $k_{1}, k_{2}$, and $k_{3}$.

The slow-roll parameters are of order $\mathcal{O}(0.01)$, so $f_{\mathrm{NL}} \sim$ $\mathcal{O}(0.01)$ for these models. Even if we start with Gaussian primordial perturbations, nonlinear effects in CMB evolution will generate $f_{\mathrm{NL}} \sim \mathcal{O}(1)$ [37], and a similar number for large scale structures due to the nonlinear gravitational evolution or the galaxy bias [35]. It seems unlikely that we can disentangle all these contaminations and detect such small primordial non-Gaussianities in the near future.

\section{Beyond the No-Go}

5.1. Inflation Model Building. The following are two examples of slow-roll potentials in the simplest inflation models that we studied in Section 4:

$$
\begin{gathered}
V_{\text {small }}=V_{0}-\frac{1}{2} m^{2} \phi^{2}, \\
V_{\text {large }}=\frac{1}{2} m^{2} \phi^{2} .
\end{gathered}
$$

The first type (95) belongs to the small field inflation models. The slow-roll conditions (11) require the potential to be flat enough relative to its height, that is, the mass of the inflaton should satisfy $m \ll H$. The second type (96) belongs to the large field inflation models. The potential also needs to be flat relative to its height, but here one achieves this by making the field range $\phi$ very large, typically $\phi \gg M_{\mathrm{P}}$. The other conditions that we listed in Section 4 should also be satisfied by these models. These are the classic examples, which exhibit algebraic simplicities and illustrate many essential features of inflation.

However, when it comes to the more realistic model building in a UV complete setup, such as in supergravity and string theory, situations get much more complicated. For example, it is natural that we encounter multiple light and heavy fields, and the potentials for them form a complex landscape. These multiple fields live in an internal space, whose structure can be very sophisticated. In string theory, some of them manifest themselves as extra dimensions and can have intricate geometry and warping. All these elements have to coexist with the inflationary background that introduces profound back-reactions.

Even with varieties of model building ingredients, it has been proven to be very subtle to construct an explicit and self-consistent inflation model. Indeed various problems have been noticed over the years in the course of the inflation model building. For example, consider the following problems.

(i) The $\eta$-Problem for Slow-Roll Inflation [52]. As we have seen, in order to have slow-roll inflation $[8,9]$, the mass of the inflaton field has to be light enough, $m \ll H$, to maintain a flat potential. However, in the inflationary background, the natural mass of a light particle is of order $H$. This can be seen in many ways, and in some ideal situations they are equivalent to each other. For example, one way to see this is to consider the coupling between the Ricci scalar and the inflaton, $\sim R \phi^{2}$. In the inflationary background $R \sim H^{2}$. Unless we have good reasons to set the coefficient of this term to be much less than one, it will give inflaton a mass of order $H$, spoiling the inflation. Another way to see this is to note that the effective potential in supergravity takes the form $V=V_{0} \exp \left(K / M_{\mathrm{P}}^{2}\right) \times$ other terms. Here schematically $K \sim \phi^{2}+\cdots$ is the Kahler potential and its dependence on $\phi$ is normalized as such to give the canonical kinetic term for $\phi$. So the first term in the expansion of $V$ is of order $V_{0} \phi^{2} / M_{\mathrm{P}}^{2} \sim H^{2} \phi^{2}$ and model independent. Therefore, either symmetry needs to be imposed or other tuning contributions introduced to solve this $\eta$-problem. 
(ii) The h-Problem for DBI Inflation [53]. DBI inflation [54] is invented to generate inflation by a different mechanism. It makes use of the warped space in the internal field space $[55,56]$. These warped space impose speed limits for the scalar field, so even if the potential is steep, the inflaton is not allowed to roll down the potential very quickly. A canonical example of warped space is

$$
d s^{2}=h(r)^{2}\left(-d t^{2}+a(t)^{2} d \mathbf{x}^{2}\right)+h(r)^{-2} d r^{2},
$$

where $r$ is the extra dimension (or internal space), $h(r)=r / R$ is the warp factor, and $R$ is the length scale of the warped space. The position of a $3+1$ dimensional brane in the $r$ coordinate is the inflaton. So the inflaton velocity is limited by the speed limit in the $r$-direction, $h^{2}$. In order to provide a speed limit that is small enough for inflation, the warp factor has to be small enough, $h \ll H R$. However one of the Einstein equations with the metric (97) takes the following form

$$
\left(\frac{d h}{d r}\right)^{2}-H^{2} h^{-2}=\frac{1}{R^{2}}+\text { other source terms, }
$$

where the second term on the left-hand side is due to the back-reaction of the inflationary spacetime. It is easy to see that the naive $h=r / R$ should be modified for $h<H R$, precisely where the inflation is supposed to happen. Without contributions from other source terms, such a deformed geometry closes up too quickly and leads to an unacceptable probe-brane back-reaction if we demand the inflaton still follow the speed limit. Therefore, either symmetry, or tuning using other source terms from the right-hand side of (98), is necessary to solve this $h$-problem. The $\eta$-problem and $h$ problem are closely related in an AdS/CFT setup.

(iii) The Field Range Bound [57, 58]. Large field inflation models require the field range to be much larger than $M_{\mathrm{P}}$. In supergravity and string theory, starting from a tendimensional theory with 10-dim Planck mass $M_{10}$, the 4-dim Planck mass $M_{\mathrm{P}}$ is obtained by integrating out the six extra dimensions,

$$
\begin{aligned}
& M_{(10)}^{8} \int d^{6} y d^{4} x \sqrt{-G_{(10)}} R_{(10)} \supset M_{(10)}^{8} V_{(6)} \\
& \quad \times \int d^{4} x \sqrt{-g_{(4)}} R_{(4)} \equiv M_{\mathrm{P}}^{2} \int d^{4} x \sqrt{-g_{(4)}} R_{(4)},
\end{aligned}
$$

where we use $L$ and $V_{(6)} \sim L^{6}$ to denote the size and volume of the extra dimensions, respectively. The field range $\Delta \phi$ often appears as the distance in the extra dimensions, $\Delta \phi \sim \Delta L \cdot M_{(10)}^{2}$, with the factor $M_{(10)}^{2}$ being the proportional coefficient. Clearly, $\Delta L \lesssim L$. If the field range manifests itself within a warped throat with a length scale $R$, we still require $R<L$, and so $\Delta L \lesssim L$. Together with $M_{\mathrm{P}}=M_{(10)}^{4} L^{3}$, we get

$$
\Delta \phi \lesssim \frac{M_{\mathrm{P}}}{\left(M_{(10)} L\right)^{2}}
$$

We further note that the microscopic length scale $L$ has to be much larger than the 10-dim Planck length $M_{(10)}^{-1}$ for the field theory to make sense. So $M_{(10)} L \gg 1$, and the field range $\Delta \phi$ in these models is generically sub-Planckian. For example, for a warped throat with charge $N,\left(M_{(10)} L\right)^{2} \gtrsim\left(M_{(10)} R\right)^{2} \sim$ $N^{1 / 2}$, we have

$$
\Delta \phi \lesssim \frac{M_{\mathrm{P}}}{\sqrt{N}}
$$

We have ignored a detailed numerical coefficient appearing on the right-hand side of (101), which is model dependent. For example, considering the volume $V_{(6)}$ to be the sum of the throat and a generic bulk volume, it is $\mathcal{O}(0.01)$ [57]; considering an extreme case where the throat does not attach to a bulk, it is $\mathcal{O}(1)$ [58]. Notice that, due to the dependence of $M_{\mathrm{P}}$ on the volume $V_{(6)}$, increasing the volume only makes the bound tighter.

(iv) The Variation of Potential [59]. Even in cases where there is no fundamental restriction on the excursion of fields, one encounters problems constructing the large field inflationary potential. Large field potentials that arise from a fundamental theory take the following general from:

$$
V(\phi)=\sum_{n=0}^{\infty} \lambda_{n} m_{\text {fund }}^{4-n} \phi^{n}
$$

where $m_{\text {fund }}$ represents typical scales in the theory. For field theory descriptions to hold, such scales are much less than $M_{\mathrm{P}}$. For example, $m_{\text {fund }}$ can be the higher dimensional Planck mass, string mass, or their warped scales. The $\lambda_{n} \mathrm{~s}$ are dimensionless couplings of order $\mathcal{O}(1)$. Unless some symmetries are present to forbid an infinite number of terms in (102), or a high degree of fine-tuning is assumed, the shape of potential (102) varies over a scale of order $m_{\text {fund }} \ll$ $M_{\mathrm{P}}$. This variation is too dramatic for the potential to be a successful large field slow-roll potential.

None of the arguments in the above list is meant to show that the specific type of inflation is impossible. In fact, these have been the driving forces for the ingenuity and creativity in the field of inflation model building. This list is used to demonstrate some typical examples of complexities in reality. Often times, solving one problem will be companied by other structures that make the model step beyond the simplest one. So we may want to keep an open mind that the algebraic simplicity may not mean the simplicity in Nature.

Following is a partial list of possibilities that allow us to go beyond the no-go theorem in Section 4.

(i) Instead of single field inflation, we can consider quasisingle field or multifield inflation models (Sections 7 and 8 ).

(ii) Instead of canonical kinetic terms, there are models where the higher derivative kinetic terms dominate the dynamics (Section 6.1).

(iii) Instead of following the attractor solution such as the slow-roll precisely, features can be present in the potentials or internal space, that temporarily break the attractor solution, or cause small but persistent perturbations on the background evolution (Sections 6.2 and 6.3$)$. 
(iv) Instead of staying in the Bunch-Davies vacuum, other excitations can exist due to, for example, boundary conditions or low scales of new physics (Section 6.4).

(v) Although strong constraints, from experimental results and theoretical consistencies, exist on nonEinstein gravities, early universe may provide an opportunity for their appearance. We use this category to include a variety of possibilities, such as modified gravities, noncommutativity, nonlocality and models beyond field theories.

There are also strong motivations from data analyses for us to search and study different large non-Gaussianities. The signal-to-noise ratio in the $\mathrm{CMB}$ data is not large enough for us to detect primordial non-Gaussianities modelindependently. A well-established method is to start with a theoretical non-Gaussian ansatz, and construct optimal estimators that compare theory and data by taking into accounts all momenta configurations. This then gives constraints on the parameters characterizing the theoretical ansatz. Therefore, the following two important possibilities exist. First, the primordial non-Gaussianities exist in data could be missed if we did not start with a right theoretical ansatz. Second, even if a non-Gaussian signal was detected with one ansatz, it does not mean that we have found the right one. So different well-motivated non-Gaussian templates are needed for clues on how corresponding data analyses should be formed. From a different perspective, even if the primordial density perturbations were Gaussian, we would still do the similar amount of work and reach the conclusion after various well-motivated non-Gaussian forms are properly constrained.

5.2. Shape and Running of Bispectra. In this paper, we will be mainly interested in the three-point correlation functions of the scalar primordial perturbation $\zeta$. They are also called the bispectra. In this subsection, we introduce some simple terminologies that we often encounter in studies of bispectra.

The three-point function is a function of three momenta, $\mathbf{k}_{1}, \mathbf{k}_{2}$, and $\mathbf{k}_{3}$, which form a triangle due to the translational invariance. Assuming also the rotational invariance, we are left with three variables, which are their amplitudes, $k_{1}, k_{2}$, and $k_{3}$, satisfying the triangle inequalities. The information is encoded in a function $S\left(k_{1}, k_{2}, k_{3}\right)$ that we define as

$$
\left\langle\zeta^{3}\right\rangle \equiv S\left(k_{1}, k_{2}, k_{3}\right) \frac{1}{\left(k_{1} k_{2} k_{3}\right)^{2}} \widetilde{P}_{\zeta}^{2}(2 \pi)^{7} \delta^{3}\left(\sum_{i=1}^{3} \mathbf{k}_{i}\right),
$$

where $\widetilde{P}_{\zeta}$ is the fiducial power spectrum, and we fix it to be a constant $\widetilde{P}_{\zeta} \equiv P_{\zeta}\left(k_{\text {wmap }}\right)=6.1 \times 10^{-9}$, where $k_{\text {wmap }}=$ $0.027 \mathrm{Mpc}^{-1}$. We have chosen the above definition so that it can be uniformly applied to different types of bispectra that we will encounter in this paper. In the literature, different notations have been used. The differences are simple and harmless. For example, different functions such as $\mathcal{A}=$ $k_{1} k_{2} k_{3} S$ or $F=S /\left(k_{1} k_{2} k_{3}\right)^{2}$ are sometimes defined. We choose $S$ since it is dimensionless and, for scale-invariant bispectra, it is invariant under a rescaling of all momenta.
This quantity is the combination that is used to plot the profiles of bispectra in the literature any way, despite of different conventions. Also, the precise power spectrum $P_{\zeta}$ instead of $\tilde{P}_{\zeta}$ is often used in the definition (103). Here, we absorb the momentum dependence of $P_{\zeta}$ in $S$. This is because the three-point function is an independent statistic relative to the two-point. In cases where both the power spectrum and bispectrum have strong scale dependence, it is not convenient if they are defined in an entangled way.

Under different circumstances, different properties of $S$ are emphasized. The conventions involved may not always be precisely consistent with each other, since they are chosen to best describe the case at hand. Following are some typical examples.

The dependence of $S$ on $k_{1}, k_{2}$, and $k_{3}$ is usually split into two kinds.

One is called the shape of the bispectrum. This refers to the dependence of $S$ on the momenta ratio $k_{2} / k_{1}$ and $k_{3} / k_{1}$, while fixing the overall momentum scale $K=k_{1}+k_{2}+$ $k_{3}$. Several special momentum configurations are shown in Figure 2.

Another is called the running of the bispectrum. This refers to the dependence of $S$ on the overall momentum scale $K=k_{1}+k_{2}+k_{3}$, while fixing the ratio $k_{2} / k_{1}$ and $k_{3} / k_{1}$.

For bispectra that are approximately scale invariant, the shape is a more important property $[50,60]$. We will encounter such cases in Sections 6.1, 7.1, and 8.1. The amplitude, also called the size, of the bispectra is often denoted as $f_{\mathrm{NL}}$ by matching

$$
S\left(k_{1}, k_{2}, k_{3}\right) \underset{\text { limit }}{\stackrel{k_{1}=k_{2}=k_{3}}{\longrightarrow}} \frac{9}{10} f_{\mathrm{NL}} .
$$

In this case, $f_{\mathrm{NL}}$ is approximately a constant but can also have a mild running, that is, a weak dependence on the overall momentum $K[61,62]$. An index $n_{\mathrm{NG}}-1 \equiv d \ln f_{\mathrm{NL}} / d \ln k$ is introduced to describe this scale dependence. The power spectrum also has a mild running, $P_{\zeta}=\left(k / k_{0}\right)^{n_{s}-1} \widetilde{P}_{\zeta}$. In this paper, when we give explicit forms of $S$ in the approximately scale-invariant cases, for simplicity, we mostly ignore these mild scale dependence and concentrate on shapes. Shapes of bispectra have been given names according to the overall dependence of $S$ on momenta. For example, for the equilateral bispectrum, $S$ peaks at the equilateral triangle limit and vanishes as $\sim k_{3} / k_{1}$ in the squeezed triangle limit $\left(k_{3} \ll k_{1}=k_{2}\right)$. The local bispectrum peaks at the squeezed triangle limit in the form $\sim\left(k_{3} / k_{1}\right)^{-1}$, such as the two shape components in (94). To visualize the shapes, we often draw $3 \mathrm{D}$ plots $S\left(1, x_{2}, x_{3}\right)$, where $x_{2}$ and $x_{3}$ vary from 0 to 1 and satisfy the triangle inequality $x_{2}+x_{3} \geq 1$.

There are also cases where the running becomes the most important property, while the shape is relatively less important $[63,64]$. In such cases, the bispectra are mostly functions of $K$. So $\mathrm{f}_{\mathrm{NL}}$ defined in (104) has strong scale dependence. Instead, one can choose a constant $f_{\mathrm{NL}}$ to describe the overall running amplitude. We will encounter such cases in Sections 6.2 and 6.3. In these cases, the shape plot $S\left(1, x_{2}, x_{3}\right)$ may look nontrivial but this is because it does not fix $K$. 


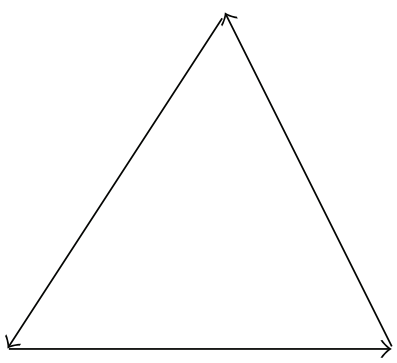

(a)

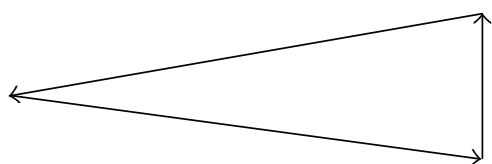

(b)

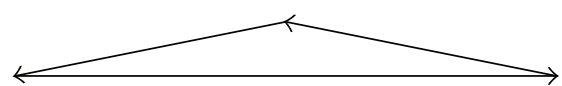

(c)

Figure 2: Momentum configurations: (a) equilateral, (b) squeezed, and (c) folded.

The above dissection will become less clean for cases where both properties become important.

One thing is clear. The $f_{\mathrm{NL}}$, that is always used to quantify the level of non-Gaussianities, is only sensible with an extra label that specifies, at least qualitatively, the profile of the momentum dependence, such as shapes and runnings.

It is useful to quantify the correlations between different non-Gaussian profiles, because as we mentioned in data analyses an ansatz can pick up signals that are not completely orthogonal to it. In real data analyses this is performed in the CMB $l$-space. To have a simple but qualitative analogue in the $k$-space, we define the inner product of the two shapes as

$$
S \cdot S^{\prime} \equiv \int_{V_{k}} S\left(k_{1}, k_{2}, k_{3}\right) S^{\prime}\left(k_{1}, k_{2}, k_{3}\right) w\left(k_{1}, k_{2}, k_{3}\right) d k_{1} d k_{2} d k_{3}
$$

and normalize it to get the shape correlator $[60,65]$

$$
C\left(S, S^{\prime}\right) \equiv \frac{S \cdot S^{\prime}}{(S \cdot S)^{1 / 2}\left(S^{\prime} \cdot S^{\prime}\right)^{1 / 2}}
$$

Following [65], we choose the weight function to be

$$
w\left(k_{1}, k_{2}, k_{3}\right)=\frac{1}{k_{1}+k_{2}+k_{3}},
$$

so that the $k$-scaling is close to the $l$-scaling in the data analyses estimator. Later in this review, when we use this correlator to estimate the correlations between shapes, we take the ratio between the smallest and largest $k$ to be $2 / 800$, close to that in WMAP. A more precise correlator should be computed in the $l$-space in the same way that the estimator is constructed. We refer to [35] for more details.

In typical data analyses [66-70], the estimator involves a triple integral of the bispectrum over the three momenta $k_{i}$. To have practical computational costs, it is necessary that this integral can be factorized into a multiplication of three integrals, each involves only an individual $k_{i}$. This requires the bispectrum to be of the form $f_{1}\left(k_{1}\right) f_{2}\left(k_{2}\right) f_{3}\left(k_{3}\right)$, or a sum of such forms. Such a form is called the factorizable form or separable form. The factor $K^{-n}$ may be tolerable since it can be written as $(1 / \Gamma(n)) \int_{0}^{\infty} t^{n-1} e^{-K t}$. If the analytical result is too complicated, to make contact with experiments we will try to construct simple factorizable ansatz or template to capture the main features of the original one. New methods that are applicable to nonfactorizable bispectrum forms and are more model-independent are under active development [71].

\section{Single Field Inflation}

In this section, we relax several restrictions of the no-go theorem on single field inflation models and study how large non-Gaussianities can arise. We present the formalisms and compute the three-point functions. We emphasize how different physical processes during inflation are imprinted as distinctive signatures in non-Gaussianities. Obviously, any mechanism that works for single field inflation can be generalized to multifield inflation models.

6.1. Equilateral Shape: Higher Derivative Kinetic Terms. In this subsection, we study large non-Gaussianities generated by noncanonical kinetic terms in general single field inflation models, following [50].

Consider the following action for the general single field inflation [72]:

$$
S=\int d^{4} x \sqrt{-g}\left[\frac{M_{\mathrm{P}}}{2} R+P(X, \phi)\right] .
$$

Comparing to (73), we have replaced the canonical form $X-$ $V$ with an arbitrary function of $X \equiv-(1 / 2) g^{\mu \nu} \partial_{\mu} \phi \partial_{\nu} \phi$ and $\phi$. This is the most general Lorentz-invariant Lagrangian as a function of $\phi$ and its first derivative. It is useful to define several quantities that characterize the differential properties of $P$ with respect to $X[49,72]$ :

$$
\begin{gathered}
c_{s}^{2}=\frac{P_{, X}}{P_{, X}+2 X P_{, X X}}, \\
\Sigma=X P_{, X}+2 X^{2} P_{, X X}=\frac{H^{2} \epsilon}{c_{s}^{2}}, \\
\lambda=X^{2} P_{, X X}+\frac{2}{3} X^{3} P_{, X X X},
\end{gathered}
$$

where $c_{s}$ is called the sound speed and the subscript " $X$ " denotes the derivative with respect to $X$. The third derivative is enough since we will only study the three-point function here.

It is a nontrivial question which forms of $P$ will give rise to inflation. The model-independent approach we take here 
is to list the conditions that an inflation model has to satisfy, no matter which mechanism is responsible for it. Namely, we generalize the slow-roll parameters in (74) to the following slow-variation parameters:

$$
\epsilon=-\frac{\dot{H}}{H^{2}}, \quad \eta=\frac{\dot{\epsilon}}{\epsilon H}, \quad s=\frac{\dot{c_{s}}}{c_{s} H}
$$

and require them to be small most of the time during the inflation. The smallness of these parameters guarantees the Hubble constant $H$, the parameter $\epsilon$, and the sound speed $c_{s}$ to vary slowly in terms of the Hubble time. Similar to the arguments given in the case of slow-roll inflation in Section 2, these are necessary to ensure a prolonged inflation as well as an approximately scale-invariant power spectrum that we observed in the CMB.

Following the same procedure that is outlined in Section 4 , we get the quadratic and cubic action for the scalar perturbation $\zeta[47,49,50]$. The quadratic part is

$$
S_{2}=\int d t d^{3} x\left[a^{3} \frac{\epsilon}{c_{s}^{2}} \dot{\zeta}^{2}-a \epsilon(\partial \zeta)^{2}\right]
$$

If the slow-variation parameters are always small and featureless, we can analytically solve the equation of motion followed from (111) and get the following mode function:

$$
u_{k}(\tau)=\frac{i H}{\sqrt{4 \epsilon c_{s} k^{3}}}\left(1+i k c_{s} \tau\right) e^{-i k c_{s} \tau}
$$

Notice the appearance of $c_{s}$ comparing to (85). The twopoint function is

$$
\left\langle\zeta\left(\mathbf{k}_{1}\right) \zeta\left(\mathbf{k}_{2}\right)\right\rangle=\frac{P_{\zeta}}{2 k_{1}^{3}}(2 \pi)^{5} \delta^{3}\left(\mathbf{k}_{1}+\mathbf{k}_{2}\right)
$$

with the power spectrum

$$
P_{\zeta}=\frac{H^{2}}{8 \pi^{2} \epsilon c_{s}}
$$

where the variables are evaluated at the horizon crossing of the corresponding $k$-mode.

To calculate the bispectrum, we look at the cubic action. In the following, we list three terms that are most interesting for this subsection,

$$
\begin{aligned}
S_{3}=\int d t d^{3} x\{ & -\frac{a^{3} \epsilon}{H c_{s}^{2}}\left[\left(1-\frac{1}{c_{s}^{2}}\right)+\frac{2 \lambda}{\Sigma}\right] \dot{\zeta}^{3} \\
& +\frac{3 a^{3} \epsilon}{c_{s}^{2}}\left(1-\frac{1}{c_{s}^{2}}\right) \zeta \dot{\zeta}^{2} \\
& \left.-a \epsilon\left(1-\frac{1}{c_{s}^{2}}\right) \zeta(\partial \zeta)^{2}+\cdots\right\} .
\end{aligned}
$$

The full terms can be found in in [50, equations (4.26)(4.28)].

The order of magnitude contribution from these three terms can be estimated similarly as we did in (92), but now we not only keep factors of $H$ and $\epsilon$, but also factors of $c_{s}$.
Take the first term as an example, we write it in terms of the conformal time,

$$
\int d \tau H_{3}(\tau) \supset \int d \tau d^{3} x \frac{a \epsilon}{H c_{s}^{2}}\left[\left(1-\frac{1}{c_{s}^{2}}\right)+\frac{2 \lambda}{\Sigma}\right] \zeta^{\prime} .
$$

Comparing (112) with (85), we see that there is an extra factor of $c_{s}$ companying $\tau$. So we estimate $d \tau \propto c_{s}^{-1}$ and $a \approx-(H \tau)^{-1} \propto c_{s} H^{-1}$. Also, $\zeta \propto H / \sqrt{\epsilon c_{s}}$, but $\zeta^{\prime} \propto c_{s} \zeta$. Overall, the vertex (116) contributes

$$
\propto \frac{H}{\sqrt{\epsilon c_{s}}}\left[\left(1-\frac{1}{c_{s}^{2}}\right)+\frac{2 \lambda}{\Sigma}\right] .
$$

Multiplying the three external legs $\zeta^{3}$, and using the definition

$$
\left\langle\zeta^{3}\right\rangle \sim f_{\mathrm{NL}} P_{\zeta}^{2}
$$

and $P_{\zeta} \propto\left(H / \sqrt{\epsilon c_{s}}\right)^{2}$, we get

$$
f_{\mathrm{NL}} \sim \mathcal{O}\left(\frac{1}{c_{s}^{2}}\right)+\mathcal{O}\left(\frac{\lambda}{\Sigma}\right) .
$$

The other two terms are similar. A detailed calculation reveals that

$$
\begin{aligned}
\left\langle\zeta\left(\mathbf{k}_{1}\right) \zeta\left(\mathbf{k}_{2}\right) \zeta\left(\mathbf{k}_{3}\right)\right\rangle= & (2 \pi)^{7} \delta^{3}\left(\mathbf{k}_{1}+\mathbf{k}_{2}+\mathbf{k}_{3}\right)\left(P_{\zeta}\right)^{2} \frac{1}{\prod_{i} k_{i}^{2}} \\
& \times\left(S_{\lambda}+S_{c}+S_{o}+S_{\epsilon}+S_{\eta}+S_{s}\right),
\end{aligned}
$$

where we have decomposed the shape of the three-point function into six parts. The first two come from the leading order terms that we listed in (115),

$$
S_{\lambda}=\left(\frac{1}{c_{s}^{2}}-1-\frac{2 \lambda}{\Sigma}\right) \frac{3 k_{1} k_{2} k_{3}}{2 K^{3}},
$$

$$
\begin{aligned}
S_{c}= & \left(\frac{1}{c_{s}^{2}}-1\right)\left(-\frac{1}{K} \sum_{i>j} k_{i}^{2} k_{j}^{2}+\frac{1}{2 K^{2}} \sum_{i \neq j} k_{i}^{2} k_{j}^{3}+\frac{1}{8} \sum_{i} k_{i}^{3}\right) \\
& \times \frac{1}{k_{1} k_{2} k_{3}} .
\end{aligned}
$$

In terms of $f_{\mathrm{NL}}$ their sizes are

$$
\begin{gathered}
f_{\mathrm{NL}}^{\lambda}=\frac{5}{81}\left(\frac{1}{c_{s}^{2}}-1-\frac{2 \lambda}{\Sigma}\right), \\
f_{\mathrm{NL}}^{c}=-\frac{35}{108}\left(\frac{1}{c_{s}^{2}}-1\right) .
\end{gathered}
$$

The next four terms come from the subleading terms that we did not list explicitly in (115) as well as the subleading contributions from the first two terms. Their orders of magnitude are

$$
\begin{gathered}
f_{\mathrm{NL}}^{o}=\mathcal{O}\left(\frac{\epsilon}{c_{s}^{2}}, \frac{\epsilon \lambda}{\Sigma}\right), \\
f_{\mathrm{NL}}^{\epsilon, \eta, s}=\mathcal{O}(\epsilon, \eta, s) .
\end{gathered}
$$

The detailed profiles can be found in [50]. 
The full results we obtained can be used in different regimes.

(i) If we look at the limit, $c_{s} \ll 1$ or $\lambda / \Sigma \gg 1$, the leading order results give two shape components, $S_{\lambda}$ and $S_{c}$. This result can also be obtained using a simple method of considering only the fluctuations in scalar field while neglecting those in gravity [73, 74]. Intuitively, this is because the higher derivative terms are responsible for the generation of large non-Gaussianities, and the gravity contribution is expected to be small as we saw in Section 4 . Therefore, one expands $P(X, \phi)$ using $\phi(\mathbf{x}, t)=\phi_{0}(t)+$ $\delta \phi(\mathbf{x}, t)$. The derivatives of $P$ with respect to $\phi$ are ignored because the inflation and scale invariance imposes an approximate shift symmetry on $P$ in terms of inflaton $\phi$. We then get two terms in the cubic Lagrangian density,

$$
\begin{aligned}
\mathcal{L}_{3}= & a^{3}\left(\frac{1}{2} P_{, X X} \dot{\phi}_{0}+\frac{1}{6} P_{, X X X} \dot{\phi}_{0}^{3}\right) \dot{\delta} \phi^{3} \\
& -\frac{a}{2} P_{, X X} \dot{\phi}_{0} \dot{\delta} \phi(\nabla \delta \phi)^{2} .
\end{aligned}
$$

This gives two leading bispectra the same as (121) and (122). The approach that we present here gives a rigorous justification to such an method. The subleading order component $S_{o}$ may be observable as well. At this limit where the higher derivative terms of the inflaton field are dominant, the Lagrangian of the above effective field theory are generalized [75] to include, for example, the ghost inflation [76] whose Lagrangian cannot be written in a form of $P(X, \phi)$. Another two slightly different equilateral shapes arise. However, it is worth to mention that, generally in single field models and Einstein gravity, going beyond $P(X, \phi)$ requires adding either terms that explicitly break the Lorentz symmetry, or terms with higher time derivatives on $\phi$ which cannot be eliminated by partial integration, such as $(\square \phi)^{2}$. Different treatment of such terms and discussions on their effects can be found in [77-79].

(ii) If we take the opposite, slow-roll limit, $c_{s} \rightarrow 1$ and $\lambda / \Sigma \rightarrow 0$, we recover the two shape components $S_{\epsilon}$ and $S_{\eta}$ that we got in Section 4, with unobservable size $f_{\mathrm{NL}} \sim \mathcal{O}(\epsilon)$.

(iii) We can also look at the intermediate parameter space. In slow-roll inflation models, one can also add higher derivative terms $[49,80]$. But in order not to spoil the slow-roll mechanism, the effect of these terms can only be subdominant. This corresponds to $c_{s} \approx$ 1 and $\lambda / \Sigma<\mathcal{O}(1)$. Using the full results, we can see that the size of the non-Gaussianity is $f_{\mathrm{NL}}<$ $\mathcal{O}(1)$. Therefore, it is important to emphasize that, for the class of models we consider here, nonslowroll inflationary mechanisms, such as the example that will be given below, are necessary to generate observable large non-Gaussianities.

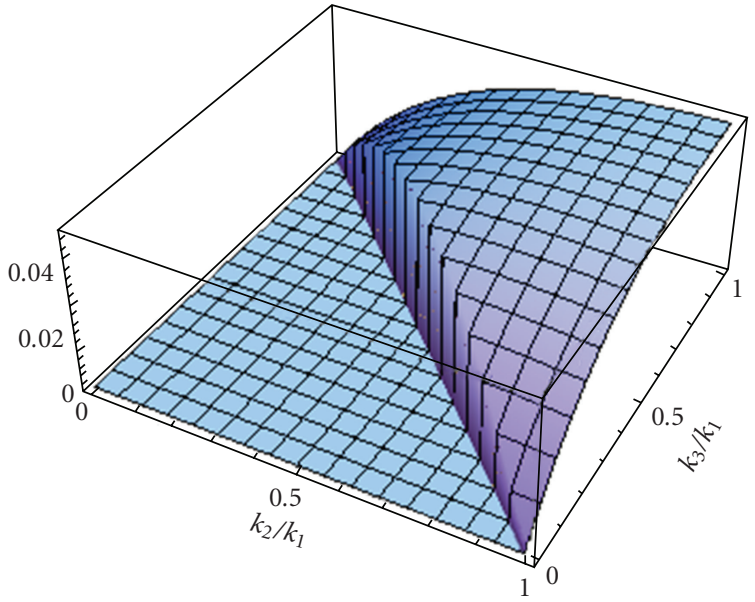

Figure 3: Shape of $S_{\lambda}$ in (121).

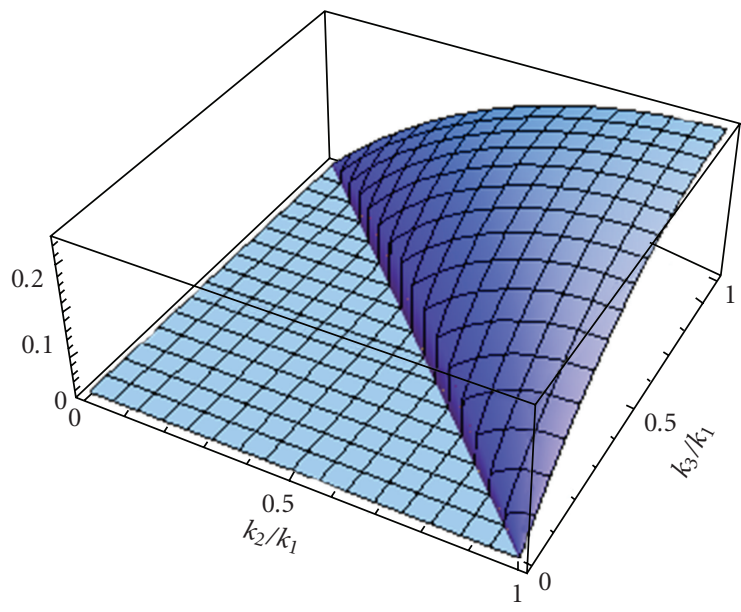

FIgURE 4: Shape of $S_{c}$ in (122).

(iv) The other terms that we did not list in (115) (see [50]) and their canonical limit (80) are also useful. These terms are exact for arbitrary values of $\epsilon, \eta$, and $s$, so the usage of the action is beyond the category of models that we focus on in this subsection. As we will see in Sections 6.2 and 6.3, it can be applied to the cases of sharp or periodic features where these parameters do not always remain small.

In the rest of this subsection, we focus on the first case.

In Figures 3 and 4 , we draw the shapes of $S_{\lambda}$ and $S_{c}$. The two shapes are similar. They both peak at the equilateral limit, and behave as $S \sim k_{3} / k_{1}$ in the squeezed limit $k_{3} \ll$ $k_{1}=k_{2}$. We call these shapes the equilateral shapes. There are some small differences between $S_{\lambda}$ and $S_{c}$, for example, around the folded triangle limit $k_{2}+k_{3}=k_{1}$. A factorizable shape ansatz for the equilateral shape that is often used in data analyses is the following [81]:

$$
S_{\text {ansatz }}^{\text {eq }}=-6\left(\frac{k_{1}^{2}}{k_{2} k_{3}}+2 \text { perm. }\right)+6\left(\frac{k_{1}}{k_{2}}+5 \text { perm. }\right)-12,
$$




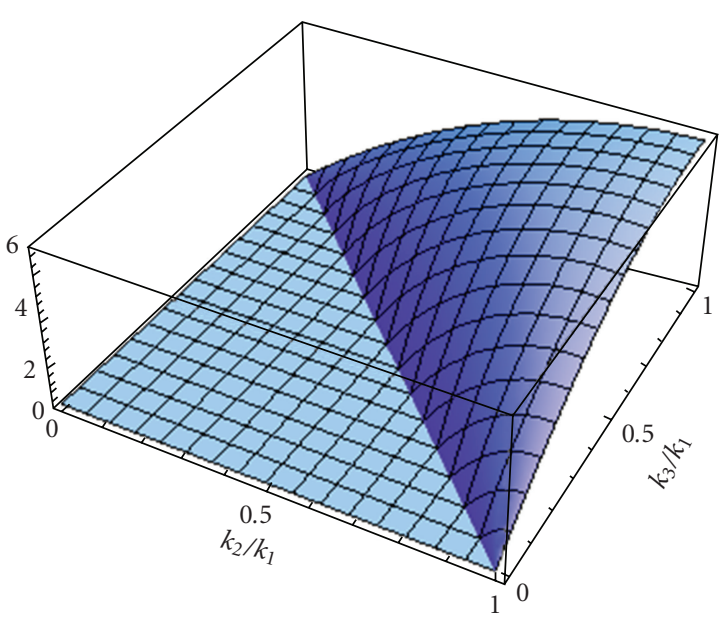

Figure 5: An ansatz (127) for the equilateral shape.

and is shown in Figure 5. As we can see, it represents the most important features of Figures 3 and 4.

The shape of $S_{o}$ is more complicated, but we expect they have the similar shapes as the equilateral one because their squeezed limits behave the same [50]. The three other shapes $S_{\epsilon}, S_{\eta}$ and $S_{s}$ are all close to the local shapes as their squeezed limit scale as $k_{1} / k_{3}$ for $k_{3} \ll k_{1}=k_{2}$.

The scale dependence in $P_{\zeta}, c_{s}$ and $\lambda / \Sigma$ will introduce mild running for the three-point function. We usually regard only the contributions from $c_{s}$ and $\lambda / \Sigma$ as the running of the non-Gaussianity.

The underlying physics of the equilateral shape can be readily understood in terms of their generation mechanism. In single field inflation, the long wavelength mode that exits the horizon are frozen and can have little interaction with modes within the horizon. The large interaction only occurs among modes that are crossing the horizon at about the same time. These modes then have similar wavelengths. This is why the shape of the non-Gaussianity peaks at the equilateral limit in momentum space.

This physical origin also suggests the caveat that, as long as there are large interactions involving modes with similar wavelengths, an equilateral-like shape may arise. For example, such cases can happen in multifield models where there are particle creation $[82,83]$ (see however [84]).

(i) An Example: Dirac-Born-Infeld (DBI) Inflation. An explicit example of the above general results is the DBI inflation [54, 73, 85-92]. These inflation models describe a 3 +1 dimensional brane moving in warped extra dimensions. The location of the brane is a scalar field in $4 \mathrm{D}$ effective field theory, and it is the inflaton. The warped extra dimensions provide a nontrivial internal field space for the inflaton. In terms of the $4 \mathrm{D}$ effective field theory, the action is

$$
\begin{aligned}
& -\int d^{4} x \sqrt{-g} \\
& \quad \times\left[f(\phi)^{-1} \sqrt{1+f(\phi) g^{\mu \nu} \partial_{\mu} \phi \partial_{\nu} \phi}-f(\phi)^{-1}+V(\phi)\right] .
\end{aligned}
$$

The nontrivial part is the kinetic term involving the squareroot. It can be understood as a generalization of the following two familiar situations. It is a higher dimensional generalization of the action of a relativistic point particle

$$
\int d t \sqrt{1-f \dot{\mathbf{x}}^{2}}
$$

where the speed of light $f^{-1 / 2}$ varies with $\mathbf{x}$. It is also a relativistic generalization of the usual canonical kinetic term in the nonrelativistic limit $\left|f(\phi) g^{\mu \nu} \partial_{\mu} \phi \partial_{\nu} \phi\right| \ll 1$,

$$
-\int d^{4} x \sqrt{-g}\left[\frac{1}{2} g^{\mu \nu} \partial_{\mu} \phi \partial_{\nu} \phi+V(\phi)\right] .
$$

Because the speed limit of the inflaton $f^{-1 / 2}$ can vary in the internal space, if it can be made small enough near the top of potential where the inflaton is about to roll down, the warped space restricts the rolling velocity even if the potential is too steep for slow-roll inflation to happen. So the inflaton rolls ultra relativistically, but with very small velocity, and this generates the DBI inflation.

The physical consequence is now easy to obtain using the general results in this subsection. In our notation the Lagrangian is

$$
P=-f^{-1} \sqrt{1-2 f X}+f^{-1}-V .
$$

The sound speed is

$$
c_{s}=\sqrt{1-2 f X}
$$

which is the inverse of the Lorentz boost factor $\gamma$, so $c_{s} \ll$ 1. The component (123) vanishes identically, and we have a large bispectrum of shape $\mathcal{A}_{c}$ with size (124).

DBI inflation is still driven by the potential energy. The general single field inflation models also include the $\mathrm{k}$ inflation [93], where the inflation is driven by the inflaton kinetic energy. Model construction of single field k-inflation can be found in [93-95]. The bispectra for such models are computed in $[50,94]$.

Multifield generalization have been studied in [96-102], where this type of kinetic terms are generalized to multiple fields. The three-point functions involving these different fields have the same or similar shapes.

The current $\mathrm{CMB}$ constraint on the equilateral ansatz $(127)$ is $-214<f_{\mathrm{NL}}^{\mathrm{eq}}<266[1]$.

6.2. Sinusoidal Running: Sharp Feature. Although various slow-variation parameters in (110) have to be small most of the time during inflation, they can become temporarily large. Such cases can happen if there are sharp features in inflaton potentials or internal field space, so the behavior of inflatons temporarily deviates from the attractor solution, and then relaxes back within several Hubble time, or stay longer but with small deviation amplitudes. Motivations for such models include the following. It may be possible explanations for features in power spectrum [103-106], and if so the associated non-Gaussianity is a cross-check. And there are brane inflation models that are very sensitive to 
sharp features present in the potential or in the internal space [107].

As an example, we study a sharp feature in the slowroll potential. The fact that a sharp feature in potential can enhance non-Gaussianities has long been anticipated and qualitative estimates have been made by different methods [108-110]. The precise method of analyzing the size, running and shape of such non-Gaussianities $[63,64]$ is made possible with the developments of the formalisms that we reviewed in Sections 3.1, 4, and 6.1. This will be the subject of this subsection.

We start by studying the behavior of the slow-roll parameters. We use a small step in potential as an example and will ignore numerical coefficients. We use $c \sim \Delta V / V$ to denote the relative height of the step, and $d$ the width of the step. In the attractor solution, the inflaton velocity is given by $\dot{\phi} \sim V^{\prime} / H \sim \sqrt{\epsilon V}$. As it falls down the step, the potential energy $c V$ gets converted to the kinetic energy, so we have

$$
\dot{\phi} \lesssim \sqrt{V(c+\epsilon)}
$$

The amplitude of density perturbations is given by $P_{\zeta} \sim$ $H^{4} / \dot{\phi}^{2}$, so such a sharp feature causes glitches in the power spectrum. It will leave a dip with relative size $\Delta P_{\zeta} / P_{\zeta} \sim$ $\sqrt{1+c / \epsilon}-1$ since $\dot{\phi}$ increases first, followed by oscillations caused by a nonattractor component of the mode function before it settles down again in the attractor solution. To fit the $\mathrm{CMB}$ data, $\dot{\phi}$ cannot change much. As we can see, the sensitivity of the power spectrum to the step size $c$ is proportional to $\epsilon$, and we need $c / \epsilon \lesssim 1$. Reducing the width $d$ of sharp feature increases the amplitude of the glitches, but this is only for a large $d$ over which the inflaton spends more than one e-fold to cross. Further reducing $d$ will not change the amplitude of the glitches since (133) is saturated, but the sharpness will determine how deep within the horizon the modes are affected.

So $\epsilon$ does not change much, $\Delta \epsilon \sim \Delta\left(\dot{\phi}^{2}\right) / H^{2} \sim c$. But it changes within a very short period, $\Delta t \sim \Delta \phi / \dot{\phi} \sim$ $d / \sqrt{V(c+\epsilon)}$. So $\eta$ can be very large,

$$
\Delta \eta \sim \frac{\Delta \epsilon}{H \epsilon \Delta t} \sim \frac{c \sqrt{c+\epsilon}}{d \epsilon} .
$$

It is also clear that the feature is associated with a characteristic physical scale and generates a scale-dependent power spectrum and higher-order correlation functions.

With these qualitative behavior in mind, we now study the three-point function. An important fact of the formalisms in Sections 4 and 6 is that the expansion is exact in terms of the slow-variation parameters. So it is valid even if these parameters are not always small, as long as the expansion in $\zeta \sim \mathcal{O}\left(10^{-5}\right)$ is perturbative.

In all terms in the cubic expansion $(80), \zeta$ appears at most with one time derivative; the field redefinition gives a term that is proportional to $\eta$ at the end of the inflation; and the other terms are all suppressed by powers of $\epsilon$, which remains small even in the presence of a sharp feature. So the most important term is

$$
\int d t d^{3} x \frac{1}{2} a^{3} \epsilon \dot{\eta} \zeta^{2} \dot{\zeta}
$$

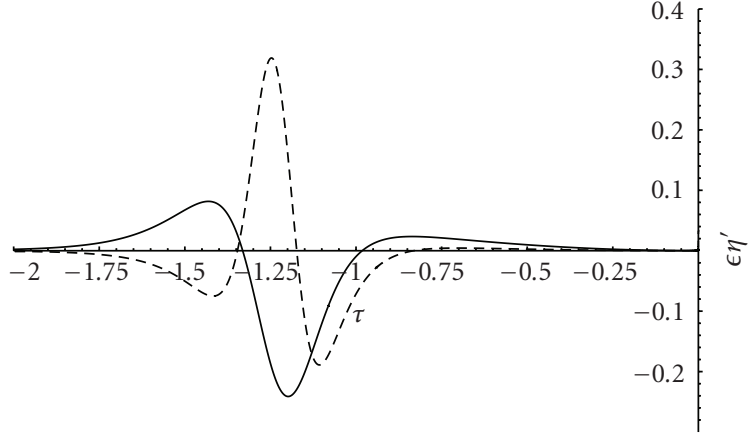

FIGURE 6: Behavior of the slow-roll parameters for a step (solid line, $c=0.0018, d=0.022 M_{\mathrm{P}}$ ) and bump (dashed line, $c=0.0005$, $\left.d=0.01 M_{\mathrm{P}}\right)$ sharp feature on $(1 / 2) \mathrm{m}^{2} \phi^{2}$ potential. Note that in the absence of the sharp feature, $\epsilon \eta^{\prime}$ is of order $\mathcal{O}\left(10^{-4}\right)$.

in which the coupling is proportional to $\dot{\eta}$. The correlation function $\left\langle\zeta\left(\mathbf{k}_{1}\right) \zeta\left(\mathbf{k}_{2}\right) \zeta\left(\mathbf{k}_{3}\right)\right\rangle$ is dominated by

$$
\begin{aligned}
& i\left(\prod_{i} u_{i}\left(\tau_{\text {end }}\right)\right) \int_{-\infty}^{\tau_{\text {end }}} d \tau a^{2} \epsilon \eta^{\prime} \\
& \quad \times\left(u_{1}^{*}(\tau) u_{2}^{*}(\tau) \frac{d}{d \tau} u_{3}^{*}(\tau)+\operatorname{sym}\right)(2 \pi)^{3} \delta^{3}\left(\sum_{i} \mathbf{k}_{i}\right)+\text { c.c. }
\end{aligned}
$$

Precise evaluation of this expression has to be done numerically. But it is not difficult to see the generic properties of bispectra associated with a sharp feature.

For long wavelength modes that already crossed the horizon at the time of the sharp feature, $k_{i} \tau \ll 1$, the mode function is already frozen and the integration (136) gives vanishing contribution. For short wavelength modes that are still well within the horizon, the modes are not affected if their momenta are larger than the inverse of the time scale characterizing the sharpness of changes in slowroll parameters. The modes most affected are those which are near the horizon crossing. These modes are all oscillatory, $\sim e^{-i k_{i} \tau}$. As we have studied, $\eta^{\prime}$ is temporarily boosted, so it can be roughly approximated as several hat-functions that satisfy $\int d \tau \eta^{\prime}=0$. Examples of such behavior are shown in Figure 6. If we simply approximate the hat-functions by several delta-functions, $\eta^{\prime} \propto \delta\left(\tau-\tau_{*}\right)$, the integration (136) will give something like

$$
S \sim f_{\mathrm{NL}}^{\mathrm{feat}} \sin \left(\frac{K}{k_{*}}+\phi_{0}\right)
$$

where $k_{*} \equiv 1 / \tau_{*}$ is the scale corresponding to the location of feature, $\phi_{0}$ is a phase and

$$
f_{\mathrm{NL}}^{\mathrm{feat}} \sim \Delta \eta \sim \mathcal{O}\left(\frac{c \sqrt{c+\epsilon}}{d \epsilon}\right) .
$$

Comparing with the effect on the power spectrum, one can keep the size of glitches in the power spectrum small while make $f_{\mathrm{NL}}$ large, for example, by fixing $c / \epsilon$ and decreasing $d$. 


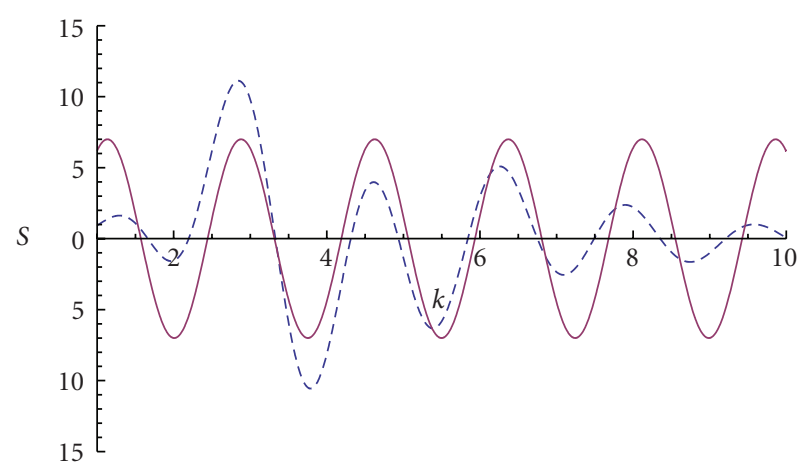

Figure 7: Numerical result (dashed line) for the bispectrum running for a sharp step $(c=0.0018, d=0.022)$ along the $k_{1}=$ $k_{2}=k_{3} \equiv k$ direction, compared with the simple ansatz (137) (solid line).

This ansatz describes the most important running behavior of this bispectrum. Notice that the oscillatory frequency in the $k$-space is of order $1 / k_{*}$, which is the scale of the feature. A rescale in $k_{*}$ can be compensated by a rescale in all $k_{i}$. Also notice that the oscillatory frequency, $3 / k_{*}$, along the $k_{1}=k_{2}=k_{3} \equiv k$ direction is $3 / 2$ of that in the power spectrum, $2 / k_{*}$. (For power spectrum, the sharp feature introduces a small non-Bunch-Davies component for the mode function. The oscillatory frequency in the power spectrum is determined by the phase of the coefficient for this component. This is obtained through matching conditions across the feature, and the phase is $\sim 2 k / k_{*}$; see Section 5.3 of [107] for an example. From this result we can see that, observationally, while sharp features located at large scales (such as $\ell \sim 30$ ) introduce glitches that need to be distinguished from statistical fluctuations, those located at much shorter scales (such as $\ell \sim 1000$ ) introduce oscillatory modulation that coherently shifts all points over several acoustic peaks in the same direction, which is completely different from statistical fluctuations of data points.)

In practice, (137) is a crude ansatz that needs to be refined. First of all, we have only considered the modes that have not exited the horizon. For those that did, as we mentioned, their correlation function is as small as usual. The ansatz needs to be cut off for the long wavelength modes $K / k_{*} \ll 1$. A more detailed analysis [64] reveals, using the hat functions as an approximation of the slowroll parameter behavior, that the bispectrum falls off as $K^{2}$ for these long wavelength modes. Secondly, the fact that in (137) all short wavelength modes are equally affected is due to the sharp-change approximation. Smoother functions will only affect a finite range of modes within the horizon. So the amplitude of the ansatz should decay and how fast depends on the sharpness of feature. To take into account both effects, empirically, we can multiply (137) with an envelope function

$$
\propto\left(\frac{K}{k_{*}}\right)^{n} e^{-K / m k_{*}}
$$

where $n$ and $m$ are parameters chosen to fit the numerical results. For example, $n=2$ and $m=5$ for Figure 7. Lastly, in the very squeezed limit, $k_{3} \ll k_{1}\left|K / k_{*}\right|, S$ can no longer be approximated as a function of $K$ only and starts to have a nontrivial shape [64]. Here we concentrate on the signature running behavior.

A numerical result with

$$
V(\phi)=\frac{1}{2} m^{2} \phi^{2}\left[1+c \tanh \left(\frac{\phi-\phi_{s}}{d}\right)\right]
$$

is shown in Figure 7. A subtlety encountered in the numerical integration is how to handle the oscillatory behavior at $\tau \rightarrow$ $-\infty$. One can do a tilt into the imaginary plane, $-\tau \rightarrow$ $-\infty(1+i \epsilon)$, as prescribed in the analytical procedure in Section 3.2; or more efficiently, perform integration by part to increase the convergence of the integrand at the $\tau \rightarrow-\infty$ end. One may also try the method of Wick rotation, but this will first require solving the background equations of motion in the Wick-rotated space, since here we do not have the analytical expression for the mode function.

Sharp features can also appear elsewhere instead of potentials, for example, in the internal warped space for DBI inflation [107]. The qualitative running behavior in bispectrum is similar, and overall large non-Gaussianities become a superposition of the approximate scale-invariant equilateral shape and the sinusoidal running.

Non-attractor initial conditions can be included as a case of sharp features, except that we only observe the relaxation part.

6.3. Resonant Running: Periodic Features. In this subsection, we consider a different type of features. These features may or may not be sharp, but the most important property is their periodicity. Such features will induce an oscillatory component to the background evolution, in particular, to the couplings in the interaction terms. We denote this oscillatory frequency as $\omega$. We know that each mode oscillates when it starts the life well within the horizon. This frequency keeps on decreasing as the mode gets stretched by the inflation, until it reaches $H$ when the mode becomes frozen. So the mode scans through all frequencies that is larger than $H$, up to some very high cutoff scale such as $M_{\mathrm{P}}$. Therefore, as long as

$$
\omega>H \text {, }
$$

the oscillatory frequency of the modes in the integral will hit $\omega$ at some point during the inflation. This causes a resonance between the couplings and modes, hence a constructive contribution to the correlation function [64]. Without the resonance, as we encountered previously, the highly oscillatory modes simply average out within the horizon. In contrast to the previous mechanisms, here the non-Gaussianities are generated when modes are subhorizon.

We now study the properties of such a non-Gaussianity, following reference [64]. 
To estimate the integral, we use the unperturbed mode function. Similar to the sharp feature case, we get

$$
\begin{aligned}
& \left\langle\zeta\left(\mathbf{k}_{1}\right) \zeta\left(\mathbf{k}_{2}\right) \zeta\left(\mathbf{k}_{3}\right)\right\rangle \\
& \approx i \frac{H^{4}}{64 \epsilon^{3} \prod_{i} k_{i}^{3}}(2 \pi)^{3} \delta^{3}\left(\sum_{i} \mathbf{k}_{i}\right) \\
& \quad \times \int_{-\infty}^{0} \frac{d \tau}{\tau} \epsilon \eta^{\prime}\left(1-i\left(k_{1}+k_{2}\right) \tau-k_{1} k_{2} \tau^{2}\right) k_{3}^{2} e^{i K \tau} \\
& \quad+\text { two perm. }+ \text { c.c.. }
\end{aligned}
$$

In this case, we are interested in the region $|K \tau| \gg 1$ in order to have resonance. So the last term dominates as long as the momentum triangle is not too squeezed so one of the $k_{i}$ s becomes $<1 / \tau$ at the resonance point. The oscillatory coupling is dominantly contributed by $\eta^{\prime}$. The integral is proportional to

$$
\int d \tau \tau \sin (\omega t) \exp (i K \tau)
$$

This integral can be done analytically using the relation $t \approx$ $-H^{-1} \ln (-H \tau)$. But its most important properties can be understood as follows in terms of the physical picture that we described.

First, let us look at its oscillatory running in $K$-space. The phase of the background repeats itself after $\Delta N_{e}=2 \pi H / \omega$ efold, during which the wave-number $K$ changes by $-K \Delta N_{e}$. So the running of the non-Gaussianity in $K$-space is also oscillatory with the period given by

$$
\Delta K=K \Delta N_{e}=\frac{2 \pi K H}{\omega} .
$$

Note that this period is changing with $K$ in a specific way that we will see more clearly in a moment.

Next, let us look at the size of the non-Gaussianity. Each $K$-mode briefly resonates with the oscillatory coupling when its frequency sweeps through the resonance frequency $\omega$. Once its frequency differs from $\omega$ by $\Delta \omega$, the integration in the 3pt starts to cancel if is performed over $\Delta t_{1} \sim \pi / \Delta \omega$. In the meanwhile it takes $\Delta t_{2} \sim \Delta \omega /(\omega H)$ to stretch the mode and change its frequency from $\omega$ to $\omega-\Delta \omega$. Equating $\Delta t_{1}$ and $\Delta t_{2}$ gives the time period over which the resonance occurs for this mode,

$$
\Delta t \sim \sqrt{\frac{\pi}{\omega H}} .
$$

This corresponds to the number of oscillation periods

$$
\frac{\omega \Delta t}{2 \pi} \sim \sqrt{\frac{\omega}{4 \pi H}}
$$

that we need to integrate over to estimate the resonance contribution. Note that one period in the integral (143) for $K / a=\omega$ contributes $\pi \tau_{*} / K$, where $\tau_{*}$ is evaluated at the resonant point. Multiplying the total number of the resonant periods (146), using the definition (103) and $\widetilde{P}_{\zeta} \approx$ $H^{2} /\left(8 \pi^{2} \epsilon\right)$, we see that the amplitude of $S\left(k_{1}, k_{2}, k_{3}\right)$ is

$$
f_{\mathrm{NL}}^{\mathrm{res}} \sim \frac{\sqrt{\pi}}{16} \eta_{A^{\prime}} \tau_{*} \sqrt{\frac{\omega}{H}} \sim \frac{\sqrt{\pi}}{8 \sqrt{2}} \frac{\omega^{1 / 2} \dot{\eta}_{A}}{H^{3 / 2}} .
$$

Slow-roll parameters acquire small oscillatory components, and here $\eta_{A}$ denotes the amplitude of such an oscillation. Other prefactors of $k_{i}$ are cancelled according the definition of $S$ and the $S$ turns out to be a function of $K$ only. In the last step of (147), we have listed the accurate numerical number, which differs from the estimate by a factor of $\sqrt{2}$.

Summarizing both the running behavior and the amplitude, we get the following ansatz for the bispectrum:

$$
S_{\text {ansatz }}^{\text {res }}=f_{\mathrm{NL}}^{\mathrm{res}} \sin \left(C \ln \left(\frac{K}{k_{*}}\right)\right),
$$

where

$$
C=\frac{2 \pi K}{\Delta K}=\frac{\omega}{H}
$$

and $k_{*}$ gives a phase. The argument $C \ln K$ in (148) appears because of (144). This gives a scale dependent oscillatory frequency in the $K$-space. In fact, this kind of dependence makes the density perturbations in the resonance model semiscale-invariant. We call it periodic-scale-invariantthey are invariant under a discrete subgroup of rescaling. Namely, the ansatz (148) is invariant if we rescale all $k_{i}$ by $n \Delta K / K=2 \pi n H / \omega$ e-fold, where $n$ is an integer. Other rescaling causes a phase shift. This property is a direct consequence of the symmetry of the Lagrangian. It is periodic, so invariant under a discrete shift of the inflaton field. This periodic-scale-invariance should also be respected by the full-bispectrum results, as well as other correlation functions.

As mentioned, we have derived this ansatz from the last term in (142). Other terms will become important in the squeezed limit. The full integration (142) has been worked out in [111], and the leading order results are

$$
\begin{aligned}
S^{\mathrm{res}}=f_{\mathrm{NL}}^{\mathrm{res}} & {\left[\sin \left(C \ln \left(\frac{K}{k_{*}}\right)\right)\right.} \\
& \left.+\frac{1}{C} \sum_{i \neq j} \frac{k_{i}}{k_{j}} \cos \left(C \ln \left(\frac{K}{k_{*}}\right)\right)+\mathcal{O}\left(\frac{1}{C^{2}}\right)\right],
\end{aligned}
$$

where $\mathcal{O}\left(1 / C^{2}\right)$ terms are neglected because we need $1 / C=$ $H / \omega \ll 1$ for large resonance. The numerical coefficient in (147) turns out to be $\sqrt{\pi} /(8 \sqrt{2})$. As we can see, the extra terms satisfy the symmetry we mentioned and indeed give large corrections in the very squeezed limit, for example, $k_{3}<k_{1} H / \omega$. These terms also ensure a consistency condition that we will study in Section 9.2. An example is plotted in Figure 8 . The spike at the very squeezed limit is due to the second term in (150). Overall, we see that the leading shape of this bispectrum is quite trivial, being almost a function of $K$ only, until it gets to the very squeezed limit. The most distinctive feature of this type of non-Gaussianities is 


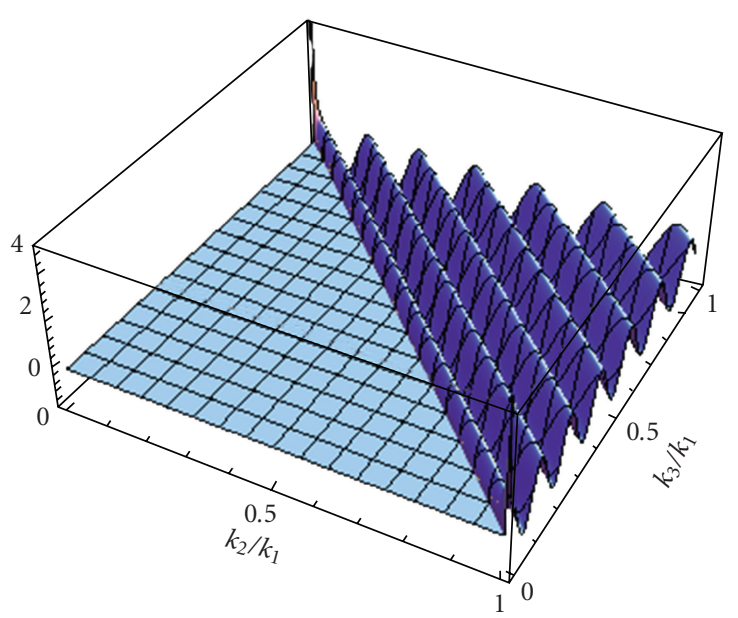

FIGURE 8: The running and shape of the resonance bispectrum (150) with $C=100$.

the running behavior captured in (148). Unfortunately, this ansatz is not factorizable if the $K$-range is too large.

More arbitrary scale-dependence can be introduced if the features are applied over a finite range, or with varying periodicity and amplitude.

As a useful comparison, the resonant running here and sinusoidal running that we studied in the last subsection are clearly distinguishable from each other observationally. The resonant running oscillates with periods that are always much smaller than the local scale, $\Delta K \ll K$; the frequency has a specific scale-dependence, $\Delta K / K=$ const.; and the frequency in the power spectrum $(\sim \sin (C \ln k)$ in $k$-space $)$ is exactly the same as that in the bispectrum $(\sim \sin (C \ln K)$ in $K$-space). In contrast, the bispectrum of the sinusoidal running oscillates with a fixed period that approximately equals to the scale at the location of the sharp feature, $\Delta K \sim k_{*}$; the frequency is scale-independent; and the power spectrum $\left(\sim \sin \left(2 k / k_{*}\right)\right.$ in $k$-space $)$ has twice an oscillatory frequency of the bispectrum ( $\sim \sin \left(K / k_{*}\right)$ in $K$-space).

As an illustration, we look at an example,

$$
V(\phi)=\frac{1}{2} m^{2} \phi^{2}\left[1+c \sin \left(\frac{\phi}{\Lambda}\right)\right]
$$

In this example, the inflaton is rolling over the small but periodic ripple laid on the potential. This induces an oscillatory component in the slow-roll parameters with an amplitude $\dot{\eta}_{A} \approx \sqrt{6} \mathrm{~cm} \phi / \Lambda^{2}$ and a frequency $\omega \approx \dot{\phi} / \Lambda \approx$ $2 \mathrm{~m} /(\sqrt{6} \Lambda)$. So we have

$$
\begin{gathered}
f_{\mathrm{NL}}^{\mathrm{res}} \sim \frac{c M_{\mathrm{P}}^{3}}{\Lambda^{5 / 2} \phi^{1 / 2}}, \\
C \approx \frac{2}{(\phi \Lambda)} .
\end{gathered}
$$

A numerical example is shown in Figure 9. As we can see, the ansatz (148) gives a very accurate fit to the actual running behavior. The mode function and power spectrum are the superposition of the usual unperturbed solution

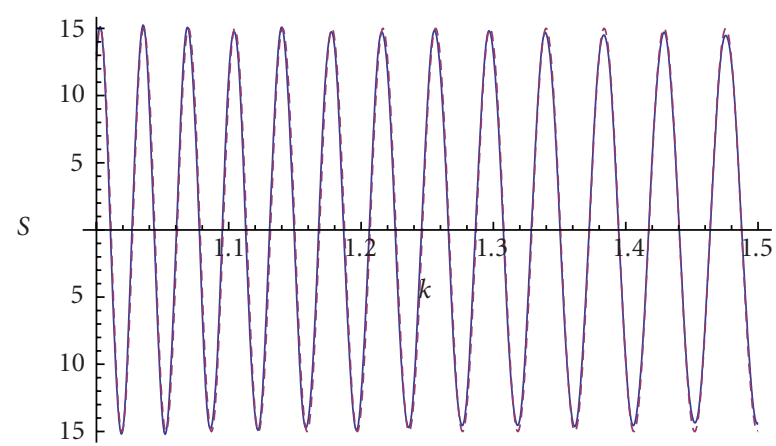

FIGURE 9: Numerical result (solid line) of the bispectrum running for the example (151) $\left(m=3 \times 10^{-6} M_{\mathrm{P}}, c=5 \times 10^{-7}, \Lambda=\right.$ $0.0007 M_{\mathrm{P}}, \phi \approx 15 M_{\mathrm{P}}$ ), compared with the ansatz (148) (dashed line).

and a small oscillatory component $[64,112,113]$. We can choose parameters so that the size of the ripples on the power spectrum is small, but bispectrum is made large. This is because the non-Gaussianities rise more quickly if we increase the frequency, while the mode function has difficulty responding efficiently when the external source oscillates too fast. This mechanism may be realized in terms of brane inflation [107] where the periodic feature comes from duality cascade in warped throat [114], or the monodromy inflation $[115,116]$ where the periodic feature comes from instanton effects $[112,113]$.

6.4. Folded Shape: A Nonstandard Vacuum. In this subsection, we study the effect of nonstandard vacuum on the primordial non-Gaussianities. We consider a different wavefunction from the Bunch-Davies vacuum when modes are well within the horizon. To start, let us first discuss several motivations for this case.

(i) A non-Bunch-Davies vacuum can actually occur much more simply than it might sound like. Any deviation from the attractor solution of the inflaton generically generates a component of non-BunchDavies vacuum. This is because a general mode function is a superposition of two components, $c_{1}(\mathbf{k}) u(\mathbf{k}, t)+c_{2}(\mathbf{k}) u^{*}(\mathbf{k}, t)$, and in attractor solution we choose one of the component asymptotic to the Bunch-Davies vacuum. A disturbance will generically introduce a mixture with the other component. In this sense, we have already encountered such a case when we studied the effect of a sharp feature in Section 6.2. Indeed, after the inflaton crosses the sharp feature, the oscillatory behavior in the power spectrum is precisely due to the superposition of the second non-Bunch-Davies component for some finite $k$-range. For an infinitely sharp change, such a disturbance with a small amplitude extends to all $k$ that have not exited the horizon at the time of sharp feature. An analytical illustration can be found in Section 5.3 of [107]. The location of the sharp feature can become superhorizon at the present 
time, but its influence has extended to much smaller scales and becomes observable. The resonance case in Section 6.3 is another type of example. An analytical illustration can be found in Section 3.3 of [112]. For non-Gaussianities studied in Sections 6.2 and 6.3 , we only concentrated on the effects caused by the features in slow-roll parameters. In analytical analyses, we approximated the mode function by the Bunch-Davies component and ignored the disturbance. The study of this subsection can be regarded as the complementary analyses on the effect of a different mode component.

(ii) In inflationary background, modes can be quantized in terms of time-dependent creation and annihilation operators, $a_{\mathbf{k}}(t)$ and $a_{-\mathbf{k}}^{\dagger}(t)$. The Bunch-Davies vacuum is defined as the vacuum annihilated by $a_{\mathbf{k}}(t)$ as $t \rightarrow-\infty$. If a different adiabatic vacuum is defined which is annihilated by $a_{\mathbf{k}}\left(t_{0}\right)$ at a finite $t_{0}$, we introduce a non-Bunch-Davies component. For example, see $[117,118]$. The origin and magnitude of such a component have been debated and studied by many papers, often under the name of the "transPlanckian effect"; see $[119,120]$ for summary and references.

(iii) There are inflation models where the scale of new physics can be very low. In particular, in warped space it is proportional to the exponentially small warp factor. In some DBI inflation models $[86,92]$, the speed limit of the inflaton and the scale of new physics are both related to the warp factor in such a way that the local warped new scale can drop near or even below the Hubble energy scale in certain epoch of inflation. Clearly the simple scalar field BunchDavies vacuum is no longer sufficient. Such models further open up the possibilities of vacuum choices.

After these discussions, let us now focus on a specific simple problem [50]. We modify the wave function of the Bunch-Davies vacuum by a small second component and examine its consequence for the three-point function calculated in Section 6.1. We consider the general single field inflation with a small sound speed $c_{s}$ or a large $\lambda / \Sigma[50,121]$.

So the mode function is

$$
u_{k}(\tau)=\frac{i H}{\sqrt{4 \epsilon c_{s} k^{3}}}\left[\left(1+i k c_{s} \tau\right) e^{-i k c_{s} \tau}+C_{-}\left(1-i k c_{s} \tau\right) e^{i k c_{s} \tau}\right]
$$

where $\left|C_{-}\right| \ll 1$ and can be $k$-dependent. In the first example above, the extra component starts at a specific time in the past. In the second class of examples, it may start either at a specific time or specific energy scale. To see a common feature without addressing these model-dependent issues, we look at the simple limit where the $\tau$ in (153) can go all the way to $-\infty$. The computation of the correlation function is essentially the same as in Section 6.1. The leading order correction to the bispectra is obtained by replacing one of the three $u_{k}(\tau)$ in the integrand by its $C_{-}$component. So

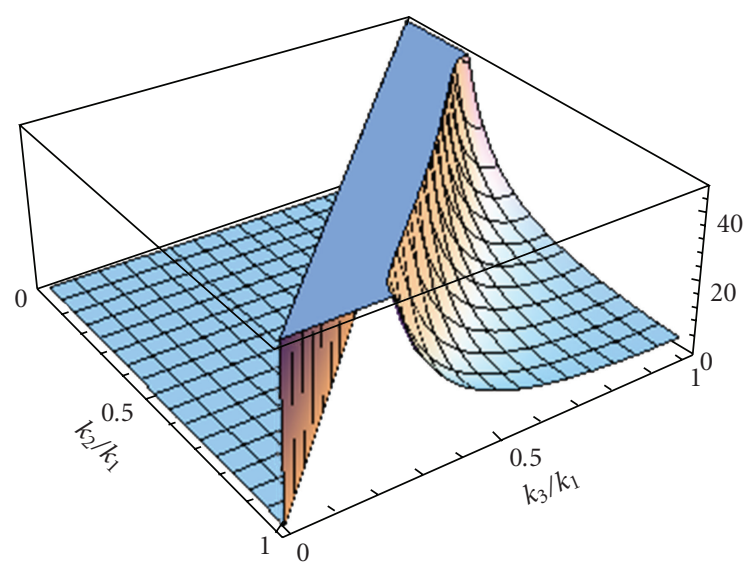

FIgURE 10: Shape of $\widetilde{S}_{\lambda}$ (truncated).

it simply replaces one of the $k_{i} \mathrm{~s}$ in the shapes with $-k_{i}$. For example, the correction to $S_{\lambda}$ is

$$
\begin{aligned}
\tilde{S}_{\lambda}= & \left(\frac{1}{c_{s}^{2}}-1-\frac{2 \lambda}{\Sigma}\right) \frac{3 k_{1} k_{2} k_{3}}{2} \\
& \times\left(\frac{\operatorname{Re}\left(C_{-}\left(k_{3}\right)\right)}{\left(k_{1}+k_{2}-k_{3}\right)^{3}}\right. \\
& \left.\quad+\frac{\operatorname{Re}\left(C_{-}\left(k_{2}\right)\right)}{\left(k_{1}-k_{2}+k_{3}\right)^{3}}+\frac{\operatorname{Re}\left(C_{-}\left(k_{1}\right)\right)}{\left(-k_{1}+k_{2}+k_{3}\right)^{3}}\right) .
\end{aligned}
$$

The shape of $\tilde{S}_{\lambda}$ is shown in Figure 10. The most important feature of this shape is the enhancement at the folded triangle limit, for example, $k_{1}+k_{2}-k_{3}=0$. The detailed form of enhancement is model dependent. For example, it is different for another shape $S_{c}$. The divergence in this folded limit occurs due to our simple limit of taking $\tau$ to $-\infty$. Imposing some kind of cutoff at the lower limit of $\tau$ will eliminate this divergence, although as mentioned the detailed modification will be highly model dependent. For example, a simple constant cutoff $\tau_{c}$ will introduce a factor of $1+\left((1 / 2) x_{c}^{2}-1\right) \cos x_{c}-x_{c} \sin x_{c}$ for each of the three terms in (154), where $x_{c} \equiv\left(k_{1}+k_{2}-k_{3}\right) c_{s} \tau_{c}$ or its cyclic. Very close to the folded limit, $\widetilde{K} c_{s}\left|\tau_{c}\right| \ll 1$ ( $\tilde{K}=k_{1}+k_{2}-k_{3}$ or its cyclic), this regulates away the divergence; away from the folded limit, $\widetilde{K} c_{s}\left|\tau_{c}\right| \gg 1$, these extra factors are unity on average but with oscillations. These oscillation can be either physical, or regarded to be zero if $x_{c}$ is within a regulation scale which exists since the nonBunch-Davies component is present for a finite time in the past.

The case for slow-roll inflation is qualitatively similar, and more examples of the bispectra shapes and the observational prospects are discussed in $[122,123]$. In this case, the proportional parameter for the bispectra amplitude is no longer enhanced by $1 / c_{s}^{2}$ or $\lambda / \Sigma$, but $<\mathcal{O}(1)$. 
In order to facilitate the data analyses, a simple ansatz has been proposed in [123],

$$
S_{\text {ansatz }, 1}^{\text {fold }}=6\left(\frac{k_{1}^{2}}{k_{2} k_{3}}+2 \text { perm. }\right)-6\left(\frac{k_{1}}{k_{2}}+5 \text { perm. }\right)+18,
$$

which represents certain important features of this kind of bispectra. It has a smooth rising behavior in the folded limit. This ansatz is plotted in Figure 11(a). Since the real shape has a model dependent cutoff, it remains open questions how sensitive this is to data analyses and how well the ansatz (155) represents it. We can also write down an ansatz which is more directly motivated from the example (154) and the comments after that equation,

$$
S_{\text {ansatz, } 2}^{\text {fold }}=k_{1} k_{2} k_{3} \frac{k_{1}+k_{2}-k_{3}}{\left(k_{c}+k_{1}+k_{2}-k_{3}\right)^{4}}+2 \text { perm., }
$$

where the cutoff scale $k_{c}=1 /\left(c_{s} \tau_{c}\right)$ is a parameter. For $k_{1}+$ $k_{2}-k_{3} \gg k_{c}$ and cyclic, we have neglected the oscillatory part and only taken the average. In this ansatz, we can change the powers in the numerator and denominator to model modeldependent variations. The relation $\left(k_{c}+k_{1}+k_{2}-k_{3}\right)^{-n}=$ $(\Gamma(n))^{-1} \int_{0}^{\infty} d t t^{n-1} e^{-\left(k_{c}+k_{1}+k_{2}-k_{3}\right) t}$ may be used to factorize the ansatz. This ansatz is plotted in Figure 11(b).

Another type of non-Bunch-Davies vacuum, namely, an $n$-particle state built on the normal Bunch-Davies vacuum, was studied in $[124,125]$ and the non-Gaussianities were found to be unobservable.

\section{Quasisingle Field Inflation}

Having considered single field inflation, we now relax the condition on the number of fields. At least during inflation, we only need to consider quantum fluctuations of light fields, since if the mass of fields are very heavy, (here the relevant scale is $m \gg H$ ), they contribute only classically and determine the classical inflaton trajectory. Multiple light fields can arise naturally if we consider the inflation models as the consequence of a UV completed framework. However, as discussed in Section 5.1, due to the back-reaction from the inflationary background, the mass of light fields are naturally of order $H$. The potential with such a shape is too steep for slow-roll inflation.

Therefore, as a natural step beyond the single field, let us consider slow-roll models with one inflationary direction, and one or more other directions that have mass neither much heavier nor much lighter than $H$. We will call the quanta in the inflationary direction as the inflaton and its mode the curvature mode, and the others isocurvaton and isocurvature modes. We call these models the quasisingle field inflation models $[46,126]$.

Note that the thematic order in this paper is not chronological. The non-Gaussianities in this type of models were not computed until very recently for a couple of reasons. If the mass of particles is of order $\mathcal{O}(H)$ or larger, the amplitude of these fields decay exponentially in time after horizon-exit. So they would not seem to

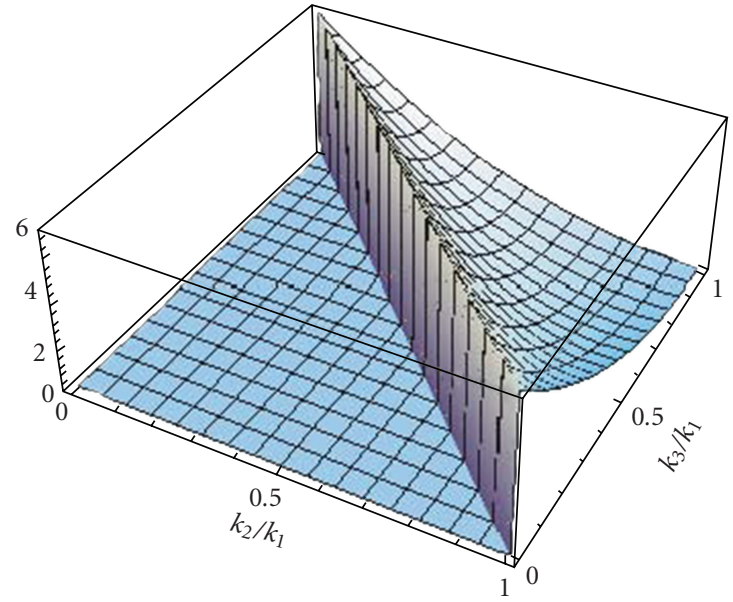

(a)

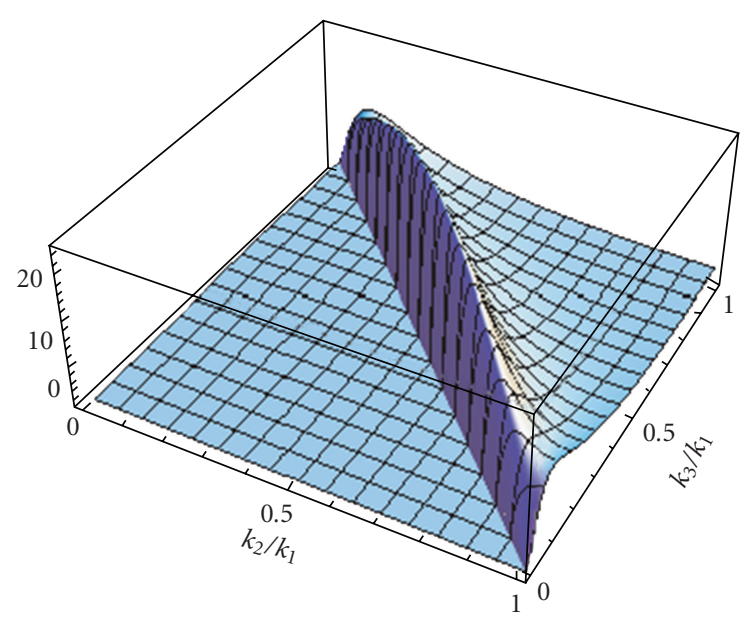

(b)

FIGURE 11: Two ansatz for the folded shape. (a) Equation (155) and (b) Equation (156) with $k_{c} / k_{1}=0.1$.

be important for superhorizon perturbations even if they couple to the curvature mode. As we will see, however, their amplitudes at or near the horizon-exit are enough to make them interesting. What really suppresses their contribution is the fast oscillation behavior present for $m \gg$ $H$. Methodologically, isocurvature-to-curvature transition for non-Gaussianities was studied restrictively in the regime of superhorizon classical evolution in multi-field space [127134], which we will explain in more details in the next section. However, for quasisingle field inflation models, a full quantum computation in the in-in formalism is necessary to properly include the contributions from both the horizon exit and the superhorizon evolution.

7.1. Intermediate Shapes: Massive Isocurvatons. There are potentially different ways massive isocurvatons can be coupled to the inflaton. We currently do not have a general approach in terms of model building. So what we will do is to first study this problem through a simple example, and 


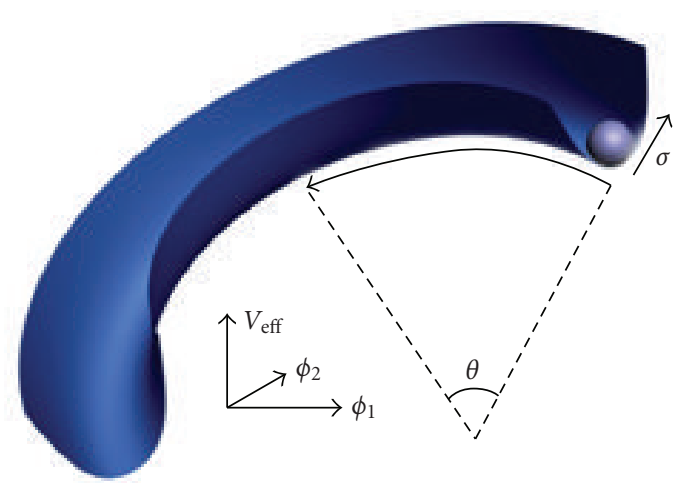

FIGURE 12: Quasi-single field inflation with turning trajectory. The field $\theta$ and $\sigma$ are in the polar coordinates. The $\theta$ is the inflationary direction with a slow-roll potential. The $\sigma$ is the isocurvature direction, which typically has mass of order $H$.

then discuss the features of the results that can be regarded as generic signatures of this class of models $[46,126]$.

We consider the case where the inflaton is turning constantly by going around (a fraction of) a circle with radius $R$ in the angular $\theta$ direction. See Figure 12. All the parameters, such as $R$ and couplings, are assumed to be constant during the turning. We call this assumption the constant turn case. In the $\theta$ direction the potential is the usual slow-roll potential $V_{\text {sr }}(\theta)$. The field in the radial direction is denoted as $\sigma$ and has mass of order $H$, and lifted by the potential $V(\sigma)$. For such a turning trajectory, it is convenient to write the action in terms of fields in the polar coordinates, $\theta$ and $\sigma$, instead of in the Cartesian coordinates,

$$
\begin{aligned}
S_{m}=\int \mathrm{d}^{4} x \sqrt{-g} & {\left[-\frac{1}{2}(R+\sigma)^{2} g^{\mu \nu} \partial_{\mu} \theta \partial_{\nu} \theta\right.} \\
- & \left.\frac{1}{2} g^{\mu \nu} \partial_{\mu} \sigma \partial_{\nu} \sigma-V_{\mathrm{sr}}(\theta)-V(\sigma)\right] .
\end{aligned}
$$

The potential $V(\sigma)$ balances off the centrifugal force necessary for the turning and traps the field at the bottom of the effective potential, $V_{\text {eff }}(\sigma)=-(1 / 2) \dot{\theta}_{0}^{2}(R+\sigma)^{2}+V(\sigma)$. We define the minimum of this effective potential to be $\sigma=0$. We expand the effective potential as

$$
V_{\text {eff }}=\text { const. }+\frac{1}{2}\left(V^{\prime \prime}-\dot{\theta}_{0}^{2}\right) \sigma^{2}+\frac{1}{6} V^{\prime \prime \prime} \sigma^{3}+\cdots,
$$

where $\dot{\theta}_{0}$ is the turning angular velocity and the primes on $V$ denote derivatives with respective to $\sigma$.

To study the perturbation theory, we perturb the fields in the spatially flat gauge,

$$
\theta(\mathbf{x}, t)=\theta_{0}(t)+\delta \theta(\mathbf{x}, t), \quad \sigma(\mathbf{x}, t)=\delta \sigma(\mathbf{x}, t),
$$

and obtain the following Hamiltonian,

$$
\begin{gathered}
\mathcal{H}_{0}=a^{3}\left[\frac{1}{2} R^{2} \delta \dot{\theta}_{I}^{2}+\frac{R^{2}}{2 a^{2}}\left(\partial_{i} \delta \theta_{I}\right)^{2}\right. \\
\left.+\frac{1}{2} \delta \dot{\sigma}_{I}^{2}+\frac{1}{2 a^{2}}\left(\partial_{i} \delta \sigma_{I}\right)^{2}+\frac{1}{2} m^{2} \delta \sigma_{I}^{2}\right] \\
\mathcal{H}_{2}^{I}=-c_{2} a^{3} \delta \sigma_{I} \delta \dot{\theta}_{I}, \\
\mathcal{H}_{3}^{I}=c_{3} a^{3} \delta \sigma_{I}^{3},
\end{gathered}
$$

where

$$
c_{2}=2 R \dot{\theta}_{0}, \quad c_{3}=\frac{1}{6} V^{\prime \prime \prime}, \quad m^{2}=V^{\prime \prime}+7 \dot{\theta}_{0}^{2}
$$

are all constants. Terms suppressed by $\mathcal{O}(\epsilon)$ have been ignored in this gauge. The curvature perturbation $\zeta$ is most transparent in another gauge, the uniform inflaton gauge, where

$$
\theta(\mathbf{x}, t)=\theta_{0}(t), \quad \sigma(\mathbf{x}, t)=\sigma_{0}(t)+\delta \sigma(\mathbf{x}, t),
$$

and the spatial metric is

$$
h_{i j}(\mathbf{x}, t)=a^{2}(t) e^{2 \zeta(\mathbf{x}, t)} \delta_{i j} .
$$

In this gauge, $\zeta$ appears in the metric as the spacedependent rescale factor and the fluctuations in the inflaton is shifted away. The relation between $\zeta$ and $\delta \theta$ is the gauge transformation. At the leading order this is

$$
\zeta \approx-\frac{H}{\dot{\theta}_{0}} \delta \theta .
$$

We will calculate the correlation functions in terms of $\delta \theta$ and then use this relation to convert them to those of $\zeta$. The fullperturbation theory that one obtains in the uniform inflaton gauge justifies the above omission of several $\mathcal{O}(\epsilon)$ terms in the spatially flat gauge [46].

There are several important points for this Hamiltonian.

First, the kinematic Hamiltonian (160) describes two free fields in the inflationary background. One is massless and has the familiar mode function,

$$
u_{\mathrm{k}}=\frac{H}{R \sqrt{2 k^{3}}}(1+i k \tau) e^{-i k \tau} .
$$

Another is massive and the mode function is

$$
v_{\mathbf{k}}=-i e^{i(v+(1 / 2))(\pi / 2)} \frac{\sqrt{\pi}}{2} H(-\tau)^{3 / 2} H_{v}^{(1)}(-k \tau),
$$

where

$$
v=\sqrt{\frac{9}{4}-\frac{m^{2}}{H^{2}}} .
$$

For $0 \leq m / H \leq 3 / 2$, the amplitude of the mode $v_{\mathbf{k}}$ decays as $(-\tau)^{-\nu+3 / 2}$ after horizon-exit $k \tau \rightarrow 0$. The lighter the isocurvaton is, the slower it decays. At the $v \rightarrow 3 / 2$ (i.e., $m / H \rightarrow 0)$ limit, the amplitude is frozen. For $m / H>$ $3 / 2, v$ becomes imaginary, the mode $v_{\mathrm{k}}$ not only contains a 
decay factor $(-\tau)^{3 / 2}$ but also an oscillation factor $\tau^{\nu}$. This oscillation is marginal for $m \sim H$, but if $m \gg H$, it causes cancellation in the integrals of the correlation function and is equivalent to factors of Boltzmann-like suppression $\sim e^{-m / H}$. We will consider the case $0 \leq v \leq 3 / 2$.

Second, there is a sharp contrast between the $V_{\mathrm{sr}}^{\prime \prime \prime}$ for the slow-roll inflaton field and the $V^{\prime \prime \prime}$ for the massive field $\sigma$ in the noninflationary direction. The former has to be very small, $\sim \mathcal{O}\left(\epsilon^{2}\right) P_{\zeta}^{1 / 2} H$, in order to maintain the smallness of the slow-roll parameters. (Here we use $\epsilon$ to denote collectively all slow-roll parameters, $\epsilon \equiv-\dot{H} / H^{2}, \eta \equiv$ $\dot{\epsilon} / \epsilon H$, and $\xi \equiv \dot{\eta} / \eta H$.) Consequently it contributes $\mathcal{O}\left(\epsilon^{2}\right)$ to the $f_{\mathrm{NL}}$ of bispectrum in slow-roll inflation, generally smaller than the $\mathcal{O}(\epsilon)$ contributions from the other terms in the same model. However, for quasisingle field inflation, the direction orthogonal to slow-roll does not have to satisfy the slow-roll conditions, and $V^{\prime \prime \prime}$ is almost unconstrained. For example, in the inflationary background, it can be of order $H$; and similarly, $V^{\prime \prime \prime \prime}$ can be of order one, and so forth. This isocurvaton self-interaction (162) becomes the source of large non-Gaussianities.

Third, the coupling between the isocurvaton and inflaton appears as a form of a two-point vertex operator in (161). We treat this term as part of the interaction Hamiltonian, and it is represented by the transfer vertex in Figure 13(a). The strength of the coupling is determined by the turning angular velocity $\dot{\theta}_{0}$ in this model. This coupling is responsible for the transformation of the isocurvature perturbations, in particular their large non-Gaussianities, to the curvature perturbation.

We calculate correlation functions corresponding to the Feynman diagrams Figure 13 in terms of the in-in formalism, which we reviewed in Section 3.1. As an illuminating example to illustrate the different advantages of the three forms of the in-in formalism, we recall from Section 3.1 that the three-point function can be written in the following forms. The original definitions (72) and (68), which we refer to as the factorized form, lead to

$$
\begin{aligned}
\left\langle\delta \theta^{3}\right\rangle= & -12 c_{2}^{3} c_{3} u_{p_{1}}^{*}(0) u_{p_{2}}(0) u_{p_{3}}(0) \\
& \times \operatorname{Re}\left[\int_{-\infty}^{0} d \tilde{\tau}_{1} a^{3}\left(\tilde{\tau}_{1}\right) v_{p_{1}}^{*}\left(\tilde{\tau}_{1}\right) u_{p_{1}}^{\prime}\left(\tilde{\tau}_{1}\right)\right. \\
& \times \int_{-\infty}^{\tilde{\tau}_{1}} d \tilde{\tau}_{2} a^{4}\left(\tilde{\tau}_{2}\right) v_{p_{1}}\left(\tilde{\tau}_{2}\right) v_{p_{2}}\left(\tilde{\tau}_{2}\right) v_{p_{3}}\left(\tilde{\tau}_{2}\right) \\
& \times \int_{-\infty}^{0} d \tau_{1} a^{3}\left(\tau_{1}\right) v_{p_{2}}^{*}\left(\tau_{1}\right) u_{p_{2}}^{*}\left(\tau_{1}\right) \\
& \left.\times \int_{-\infty}^{\tau_{1}} d \tau_{2} a^{3}\left(\tau_{2}\right) v_{p_{3}}^{*}\left(\tau_{2}\right) u_{p_{3}}^{*}\left(\tau_{2}\right)\right] \\
& \times(2 \pi)^{3} \delta^{3}\left(\sum_{i} \mathbf{p}_{i}\right)+9 \text { other similar terms } \\
& +5 \text { permutations of } \mathbf{p}_{i} .
\end{aligned}
$$

The perturbation theory here starts from the fourth order. The reorganized commutator form (69) leads to

$$
\begin{aligned}
&\left\langle\delta \theta^{3}\right\rangle=12 c_{2}^{3} c_{3} u_{p_{1}}(0) u_{p_{2}}(0) u_{p_{3}}(0) \\
& \times \operatorname{Re}\left[\int_{-\infty}^{0} d \tau_{1} \int_{-\infty}^{\tau_{1}} d \tau_{2} \int_{-\infty}^{\tau_{2}} d \tau_{3}\right. \\
& \quad \times \int_{-\infty}^{\tau_{3}} d \tau_{4} \prod_{i=1}^{4}\left(a^{3}\left(\tau_{i}\right)\right) \\
& \times a\left(\tau_{2}\right)\left(u_{p_{1}}^{\prime}\left(\tau_{1}\right)-\text { c.c. }\right) \\
& \quad \times\left(v_{p_{1}}\left(\tau_{1}\right) v_{p_{1}}^{*}\left(\tau_{2}\right)-\text { c.c. }\right) \\
& \quad \times\left(v_{p_{3}}\left(\tau_{2}\right) v_{p_{3}}^{*}\left(\tau_{4}\right) u_{p_{3}}^{*}\left(\tau_{4}\right)-\text { c.c. }\right) \\
&\left.\quad \times v_{p_{2}}\left(\tau_{2}\right) v_{p_{2}}^{*}\left(\tau_{3}\right) u_{p_{2}}^{*}\left(\tau_{3}\right)\right] \\
& \quad \times(2 \pi)^{3} \delta^{3}\left(\sum_{i} \mathbf{p}_{i}\right)+2 \text { other similar terms } \\
&+5 \text { permutations of } \mathbf{p}_{i} .
\end{aligned}
$$

In the IR $(\tau \rightarrow 0)$, each of the ten terms in the factorized form diverge as $\tau^{3-6 v}$ for $3 / 2>v>1 / 2(0<m<\sqrt{2} H)$; while in the commutator form, various subtractions off the complex conjugates and the requirement that the final result has to be real makes such divergence explicitly disappear.

In the UV $(\tau \rightarrow-\infty)$, each factor of the multiple integral that integrates from $-\infty$ to 0 has a definite convergent direction if we choose one of the two contour tilts, $\tau_{i} \rightarrow$ $-\infty(1 \pm i \epsilon)$, accordingly. Or more efficiently, by a Wick rotation $\tau_{i} \rightarrow \pm i z_{i}$. This would have been the case for the commutator form if we can break up the integrand into individual terms. However in order to achieve the explicit IR convergence, as we saw above, these terms have to be grouped; but then they have contradicting convergence directions.

To take advantage of both forms, we introduce a cutoff $\tau_{c}$, and write the IR part $\left(\tau_{c}<\tau \leq 0\right)$ of the integrals in terms of the commutator form, and the UV part $\left(\tau<\tau_{c}\right)$ in terms of the factorized form, in the following mixed form:

$$
\begin{aligned}
& \sum_{i} \int_{\tau_{c}}^{0} d \tau_{1} \cdots \int_{\tau_{c}}^{\tau_{i-1}} d \tau_{i}\{\text { commutator form }\} \\
& \left.\quad \times \int_{-\infty}^{\tau_{c}} d \tau_{i+1} \cdots \int_{-\infty}^{\tau_{n-1}} d \tau_{n} \text { \{factorized form }\right\}
\end{aligned}
$$

This shows explicitly both convergence behavior of the correlation function. Combining with Wick-rotations of the integration contours in the UV, this form provides an efficient way to evaluate the correlation functions numerically. The shapes of bispectra are presented in Figure 14 for $v=$ $0,0.3,0.5,1$. 


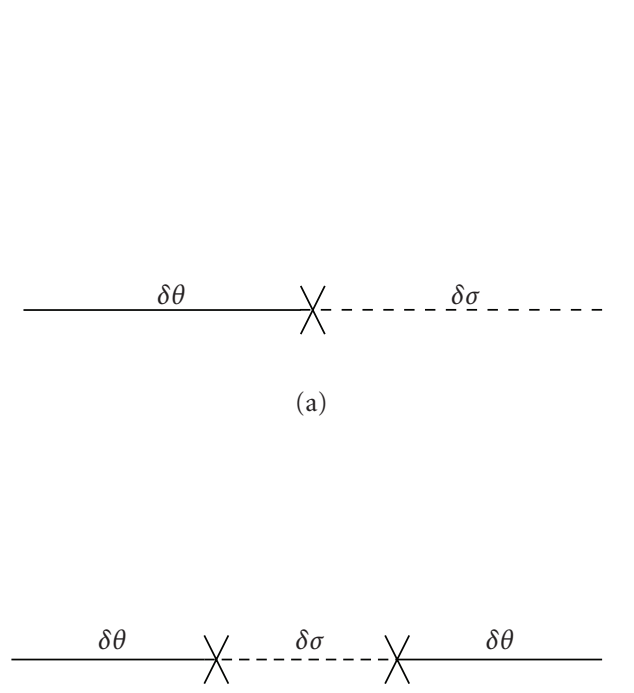

(b)

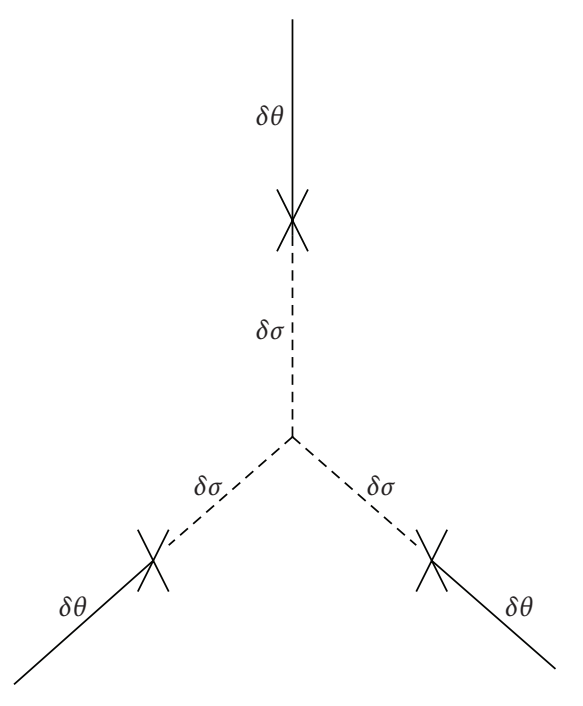

(c)

FIGURE 13: Feynman diagrams for the transfer vertex (a), corrections to the power spectrum from isocurvature modes (b), and the leading bispectrum (c).

To better understand the shapes analytically, we can work out the squeezed limit $\left(p_{3} \ll p_{1}=p_{2}\right)$ of the three-point function,

$$
\left\langle\delta \theta^{3}\right\rangle \longrightarrow \frac{c_{2}^{3} c_{3}}{H R^{6}} \frac{1}{p_{1}^{(7 / 2)-v} p_{2} p_{3}^{(3 / 2)+\nu}} s(\nu)(2 \pi)^{3} \delta^{3}\left(\sum_{i} \mathbf{p}_{i}\right)
$$

where $s(\nu)$ is a $\nu$-dependent numerical number.

Recall that the squeezed limit of $S$ for the equilateral shape goes as $p_{3} / p_{1}$, while for the local shape $\left(p_{3} / p_{1}\right)^{-1}$. Both the numerical results in Figure 14 and the analytical results in (173) show that here we have a one-parameter family of shapes, $\sim\left(p_{3} / p_{1}\right)^{1 / 2-\nu}$, lie between the two. We call them the intermediate shapes.

The physical origin of such shapes can be understood as follows, and should be a generic signature for the quasisingle field inflation models. As we have seen, the large equilateral non-Gaussianity arises because the interacting modes cross the horizon around the same time. The shape of bispectrum peaks at the equilateral limit where the modes all have comparable wavelengths. As we will see in Section 8, the large local non-Gaussianity arises due to the classical nonlinear evolution of superhorizon modes in the multifield space; so the interactions are causally disconnected and behave local in position space. This is nonlocal from the momentum space point of view. So the shape of bispectrum peaks at the squeezed limit. Now for quasisingle field inflation, the large non-Gaussianities come from the massive isocurvaton. Depending on the mass, these modes either decay right away after they exit the horizon (for $m>\sqrt{2} H$ ), or survive for a long time at the superhorizon scales (for $m<\sqrt{2} H$ ). In the former case, the generation and transfer of non-Gaussianities maximize for modes that are exiting the horizon around the same time, resulting in quasiequilateral shapes; in the latter case, the generation and transfer of non-Gaussianities happen in a superhorizon fashion, resulting in quasilocal shapes. In this regard, let us look more closely at the special limit $m / H \rightarrow 0(\nu \rightarrow 3 / 2)$.

In this massless limit, an infrared cutoff to the integrals are necessary. Otherwise the transfer will last forever for the constant turn case. The cutoff corresponds to the ending of the turning. Let us discuss the following two cases. First, we still keep $V^{\prime \prime \prime}$ large. Our analyses still apply in this case. Interestingly, the shape of the bispectrum goes to that of the local form in this limit. As we will explain in Section 8.1, this is a generic signature of a massless isocurvaton. The infrared e-fold cutoff will introduce some running in the $f_{\mathrm{NL}}^{\text {int }}$ because different modes experience different turning e-folds. Second, we would like to make the isocurvature directions flat so this becomes a two-field slow-roll inflation models. Such models were intensively studied and it is known that the isocurvature modes can be transferred to the curvature mode by turning. However, since $V^{\prime \prime \prime} \sim \mathcal{O}\left(\epsilon^{3 / 2}\right) H^{2} / M_{\mathrm{P}}$ is required to maintain the small slow-roll parameters, the contribution we computed here generates too small non-Gaussianity. We expect contributions from other terms are small as well. So it is much more difficult to generate large non-Gaussianities in such models, essentially because imposing the slow-roll conditions in all directions are too restrictive.

To connect with data analyses, guided by the numerical results and analytical squeezed limit, we can use the following ansatz to describe the full family of shapes:

$$
S_{\text {ansatz }}^{\text {int }}=\frac{3^{(9 / 2)-3 v}}{10} \frac{f_{\mathrm{NL}}^{\mathrm{int}}\left(p_{1}^{2}+p_{2}^{2}+p_{3}^{2}\right)\left(p_{1} p_{2} p_{3}\right)^{(1 / 2)-v}}{\left(p_{1}+p_{2}+p_{3}\right)^{(7 / 2)-3 v}} .
$$

These shapes are shown in Figure 15. Comparing with Figure 14, we can see that they match quite well except near 


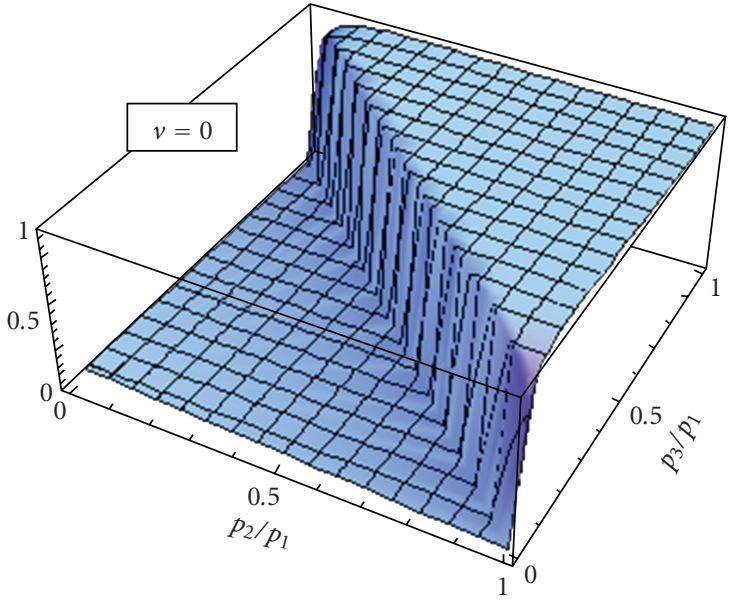

(a)

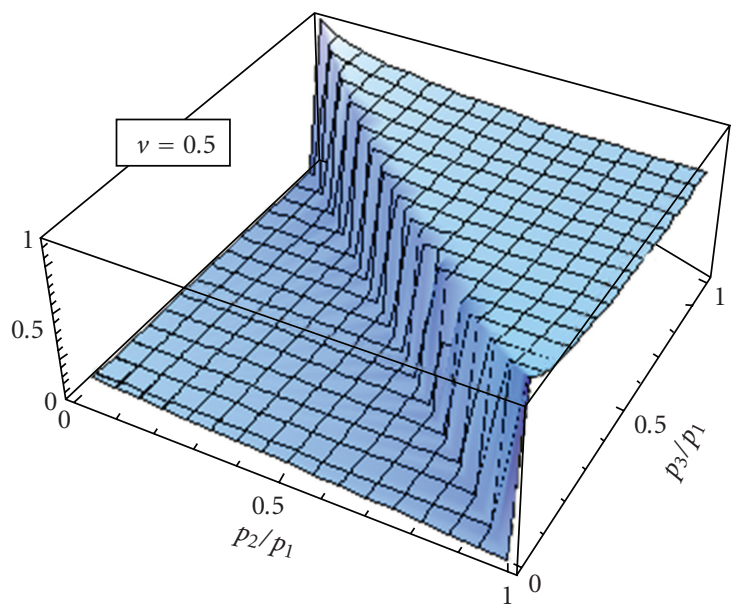

(c)

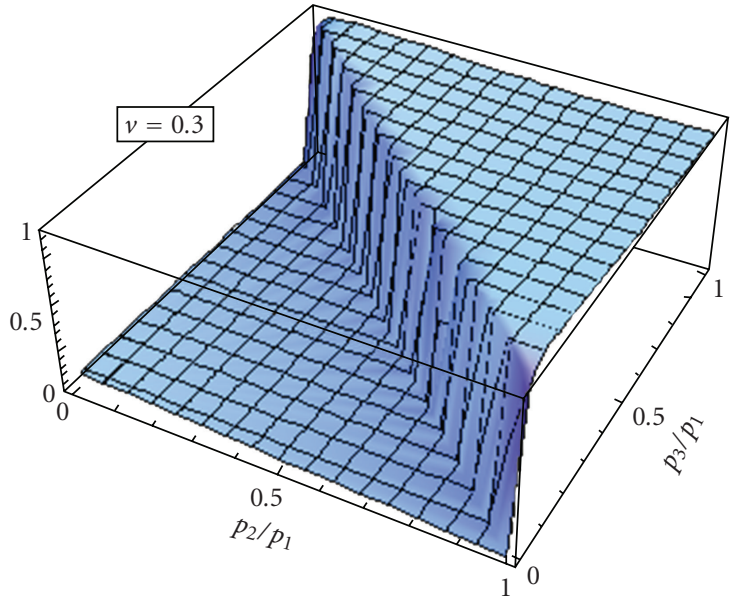

(b)

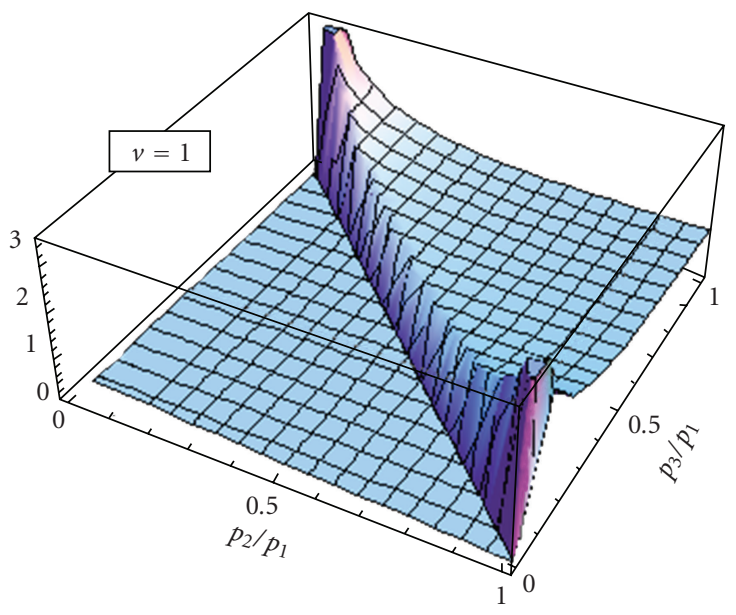

(d)

Figure 14: Numerical results for the shapes of bispectra with intermediate forms. We plot $S$ with $v=0,0.3,0.5,1$. The plot is normalized such that $S=1$ for $p_{1}=p_{2}=p_{3}$.

$\nu=0$, around which it is better represented by another ansatz in [46]. The size of this bispectrum is

$$
f_{\mathrm{NL}}^{\mathrm{int}}=\alpha(\nu) P_{\zeta}^{-1 / 2}\left(\frac{\dot{\theta}_{0}}{H}\right)^{3}\left(-\frac{V^{\prime \prime \prime}}{H}\right)
$$

where $P_{\zeta} \approx 6.1 \times 10^{-9}$ and $\dot{\theta}_{0}$ is the turning angular velocity. The $\alpha(\nu)$ is a positive numerical number which, depending on $\nu$, can give an additional enhancement factor of order $N_{f}$ ( $N_{f}$ is the turning e-folds). Since $\left(\dot{\theta}_{0} / H\right)^{2}$ and $V^{\prime \prime \prime} / H$ are the expansion parameters in the perturbation theory, they have to be small to trust our calculation. Nonetheless this is not the model-building requirement.

The fluctuations of more massive $(m>\mathcal{O}(H))$ fields may become important if they play a role later in the reheating $[135,136]$. Such cases typically require some tunings for special conditions, so that the highly suppressed fluctuation amplitude can become important.

\section{Multifield Inflation}

As we have seen in Section 7.1, if we take the isocurvaton mass to zero in quasisingle field inflation while keep the nonlinear self-couplings of the isocurvaton $V^{\prime \prime \prime}$ large, the shape of the large bispectrum in the squeezed limit approaches the local form. The local form is in fact the earliest and most well-studied example of non-Gaussianities $[66,127,137,138]$, although it was first found to be small as we have seen in Section 4. As we will explain in this section, a large local form is a signature of massless isocurvatons that have large nonlinear evolution in multifield space. We have arrived this shape from the in-in formalism by taking the massless limit. But if we stay in this limit, there is an easier formalism, the $\delta N$ formalism [139-141], in which the underlying physics of the local shape becomes transparent.

8.1. Local Shape: Massless Isocurvatons. We recall that, in single field inflation, if we use the uniform inflaton gauge where there are no fluctuations in the inflaton field, 


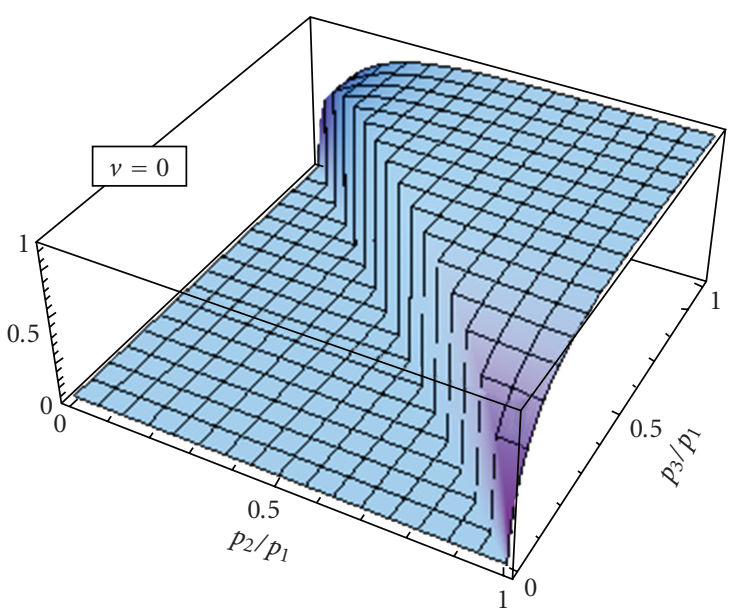

(a)

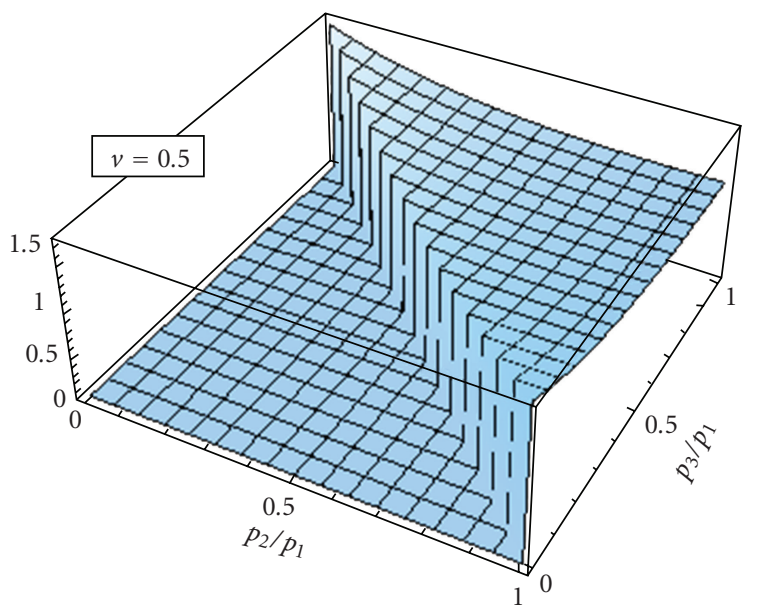

(c)

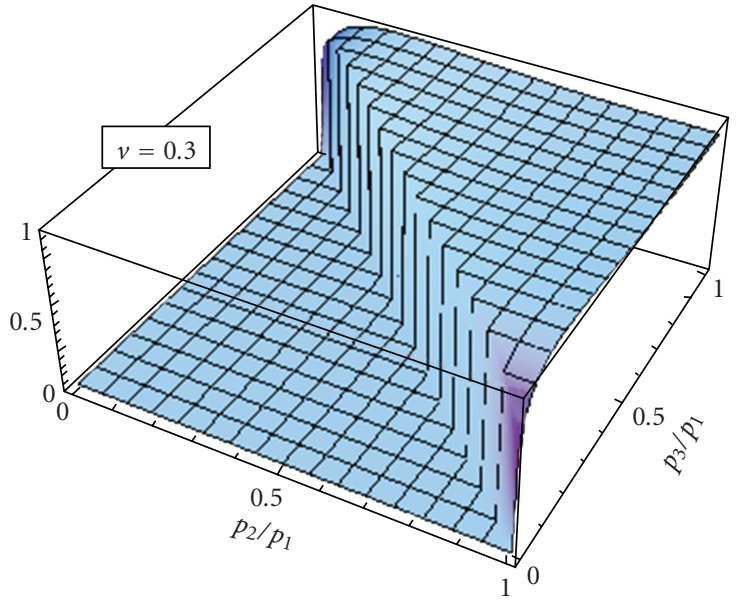

(b)

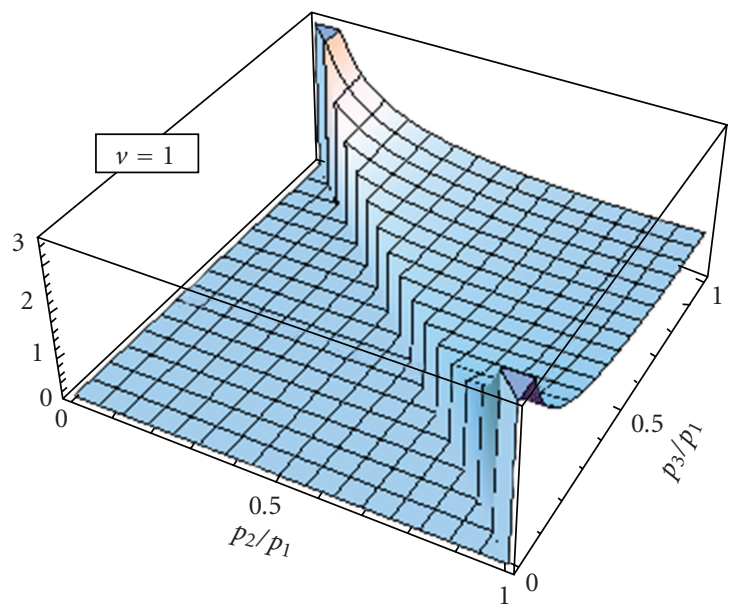

(d)

FIgURE 15: Shape ansatz (174) for the intermediate forms.

the scalar perturbation $\zeta$ enters in the scale factor as $a^{2} e^{2 \zeta}$. For superhorizon modes, $\zeta$ is frozen. If we look at the different comoving superhorizon patches, they are causally disconnected from each other. So they evolve independently and locally in space. In such a gauge, the only difference is a space-dependent scale factor. This is also called the separate universe picture. The primordial curvature perturbations manifest themselves as the different number of expansion efold, $\delta N$, at different positions.

We would like to generalize this picture to the multifield case in the following $\delta \mathrm{N}$ formalism. We will resort to a simple version of $\delta N$ formalism stated below, which is of course a consequence of the in-in formalism, but formulated from a simple perspective which clearly illustrates the points in this section. Otherwise, as we will explain, in the most general sense the $\delta N$ formalism should be defined as the inin formalism written in terms of specified gauges.

(i) We consider a set of scalars $\phi_{i}$ during inflation. Inflaton is one of them but can be different linear combinations of $\phi_{i}$ s as a function of time, and the other orthogonal fields are called the isocurvatons.
All the modes that we are eventually interested in should all have become superhorizon when the initial slice (specified below) is chosen. We look at different horizon-size patches and label them with the coarse-grained comoving coordinate $\mathbf{x}$. In the in-in formalism, the superhorizon modes behave as the c-number time-dependent background for each comoving patch. So we evolve these patches independently and classically.

(ii) We pick an initial spatially flat slice, on which there is no scalar fluctuations in the metric and all the fluctuations are in the scalar fields $\phi_{0 i}+\delta \phi_{i}(\mathbf{x})$. We assume that we know the statistics of such fluctuations.

(iii) We pick the final uniform density slices. Relative to the unperturbed and perturbed initial spatially flat slices, we have, respectively, the unperturbed and perturbed final uniform density slices. For single field inflation, these two final surfaces are the same. For multifield models, they are generally different. Such 
final slices have the properties that the universe has the same energy densities and field configurations everywhere on them. They can be chosen during either the inflation or the reheating. After that, every separated universe will have the same evolution. The only difference is the scale factor. This is the analogy of the uniform inflaton gauge in single field inflation. We study the cases where such slices exist.

(iv) We evolve the unperturbed $\phi_{0 i}$ in the initial slice classically to the unperturbed final slice, and denote the number of e-folds as $N_{0}\left(\phi_{0 i}\right)$. This is the unperturbed e-fold number. We evolve the perturbed $\phi_{0 i}+\delta \phi_{i}(\mathbf{x})$ in the initial slice classically to the perturbed final slice, and denote the number of e-folds as $N\left(\phi_{0 i}+\right.$ $\left.\delta \phi_{i}(\mathbf{x})\right)$. The difference between them

$$
\delta N=N\left(\phi_{0 i}+\delta \phi_{i}(\mathbf{x})\right)-N_{0}\left(\phi_{0 i}\right)
$$

is the curvature perturbation $\zeta$. Here, $N_{0}$ is a constant that can be shifted to make $\langle\delta N\rangle$ zero.

(v) We expand

$$
\delta N=N_{i} \delta \phi_{i}+\frac{1}{2} N_{i j} \delta \phi_{i} \delta \phi_{j}+\cdots
$$

where the subscripts on $N$ denote the partial derivatives with respect to $\phi_{i}$. For example, $N_{i j}=$ $\left(\partial N / \partial \phi_{i}\right)\left(\partial N / \partial \phi_{j}\right)$. Repeated indices are summed over. The correlation functions of $\zeta$ can then be computed as the classical averages of the products, such as

$$
\begin{aligned}
\left\langle\zeta\left(\mathbf{x}_{1}\right) \zeta\left(\mathbf{x}_{2}\right)\right\rangle= & N_{i} N_{j}\left\langle\delta \phi_{i}\left(\mathbf{x}_{1}\right) \delta \phi_{j}\left(\mathbf{x}_{2}\right)\right\rangle \\
\left\langle\zeta\left(\mathbf{x}_{1}\right) \zeta\left(\mathbf{x}_{2}\right) \zeta\left(\mathbf{x}_{3}\right)\right\rangle= & \frac{1}{2} N_{i j} N_{k} N_{l} \\
& \times\left\langle\delta \phi_{i}\left(\mathbf{x}_{1}\right) \delta \phi_{j}\left(\mathbf{x}_{1}\right) \delta \phi_{k}\left(\mathbf{x}_{2}\right) \delta \phi_{l}\left(\mathbf{x}_{3}\right)\right\rangle \\
& +2 \text { perm. }
\end{aligned}
$$

(vi) We have assumed that the statistics of the $\delta \phi_{i}(\mathbf{x})$ are known on the initial slice. But this is not always easy to get. So we will consider the simple cases where this statistics can be approximated as Gaussian. Otherwise, calculating such initial statistics requires using the full quantum mechanical in-in formalism.

Most generally, one identifies $\delta N$ with the scalar curvature $\zeta$ in the uniform inflaton gauge; and the relation between $\delta N$ and $\delta \phi(\mathbf{x})$ in the $\delta N$ formalism is the gauge transformation between the uniform inflaton gauge and the spatially flat gauge. Calculating the correlation functions for $\zeta$ becomes calculating those for $\delta \phi(\mathbf{x})$ using the in-in formalism. An example is the one we have seen inSection 7.1. (vii) So far we have not used the condition that the isocurvatons are massless $(m \ll H)$. If they are massive, after horizon exit, the modes decay. So in the superhorizon classical regime, where the $\delta N$ formalism is supposed to be useful, we are back in the single field inflation. Sub- and near-horizon perturbations should be computed by the full in-in formalism. Therefore, having massless isocurvatons opens up classical multifield space in which we can have sizable $\delta N$ defined in (176).

Now let us consider the Gaussian fluctuations $\delta \phi_{i}$. From Section 2, we know that for massless scalars,

$$
\left\langle\delta \phi_{i}\left(\mathbf{k}_{1}\right) \delta \phi_{j}\left(\mathbf{k}_{2}\right)\right\rangle=\frac{H_{*}^{2}}{2 k_{1}^{3}}(2 \pi)^{3} \delta^{3}\left(\mathbf{k}_{1}+\mathbf{k}_{2}\right) \delta_{i j},
$$

where $H_{*}$ is the Hubble parameter when the corresponding mode exits the horizon. If the scalars are not exactly massless, $H$ will have a running dependence on $k_{1}$ caused by the decay of the amplitude. Using

$$
\delta N(\mathbf{k})=N_{i} \delta \phi_{i}(\mathbf{k})+\frac{1}{2} N_{i j} \int \frac{d^{3} \mathbf{k}^{\prime}}{(2 \pi)^{3}} \delta \phi_{i}\left(\mathbf{k}-\mathbf{k}^{\prime}\right) \delta \phi_{j}\left(\mathbf{k}^{\prime}\right),
$$

we get the power spectrum

$$
\left\langle\zeta\left(\mathbf{k}_{1}\right) \zeta\left(\mathbf{k}_{2}\right)\right\rangle=(2 \pi)^{5} \frac{P_{\zeta}}{2 k_{1}^{3}} \delta^{3}\left(\mathbf{k}_{1}+\mathbf{k}_{2}\right),
$$

where

$$
P_{\zeta}=\left(\frac{H_{*}}{2 \pi}\right)^{2} N_{i}^{2}
$$

and the bispectrum

$$
\begin{aligned}
& \left\langle\zeta\left(\mathbf{k}_{1}\right) \zeta\left(\mathbf{k}_{2}\right) \zeta\left(\mathbf{k}_{3}\right)\right\rangle \\
& \quad=N_{i j} N_{i} N_{j} \frac{H_{*}^{4}}{4}\left(\frac{1}{k_{1}^{3} k_{2}^{3}}+2 \text { perm. }\right)(2 \pi)^{3} \delta^{3}\left(\sum \mathbf{k}_{i}\right) .
\end{aligned}
$$

According to the definition (103), the shape function is

$$
S^{\text {loc }}=\frac{3}{10} f_{\mathrm{NL}}^{\mathrm{loc}}\left(\frac{k_{1}^{2}}{k_{2} k_{3}}+2 \text { perm. }\right),
$$

where

$$
f_{\mathrm{NL}}^{\mathrm{loc}}=\frac{5}{6} \frac{N_{i j} N_{i} N_{j}}{\left(N_{l}^{2}\right)^{2}}
$$

As usual, we have ignored a mild running from the power spectrum $P_{\zeta}$. The shape (184) is called the local shape, which we plot in Figure 16. This form is already factorizable.

The physics of this shape can be understood from the derivation above. As explicitly demonstrated in (176)-(178), this non-Gaussianity is generated locally in position space for superhorizon modes. After Fourier transform, it becomes nonlocal in momentum space. That is the reason that the shape peaks at the squeezed limit.

If the perturbation $\delta \phi(\mathbf{x}, t)$ on the initial spatially flat slice cannot be approximated as Gaussian, the shapes of final bispectra can be more complicated. 


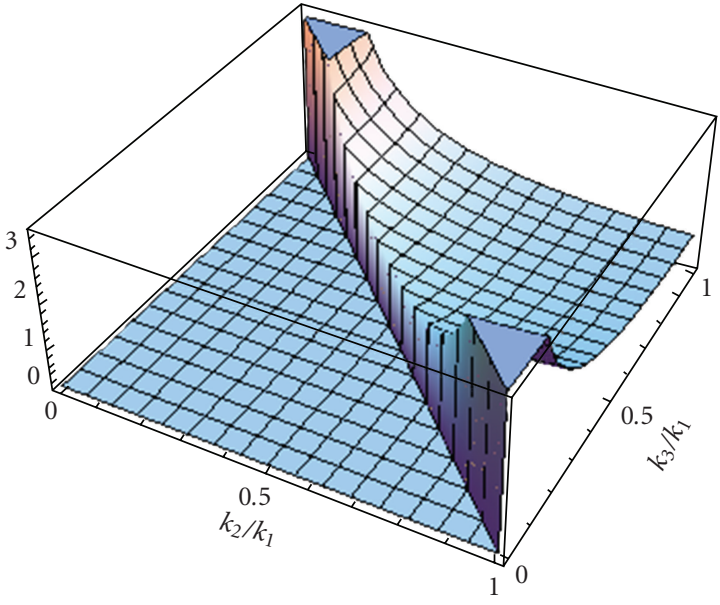

FIgURE 16: Shape of the local form (184).

(i) An Example: The Curvaton Model. We use the curvaton model [142-151] as an example to illustrate the generation of large local non-Gaussianity. We also use it to demonstrate the $\delta N$ formalism.

In this model, we assume that during inflation there is another light field $\sigma$ with the potential

$$
V(\sigma)=\frac{1}{2} m_{\sigma}^{2} \sigma^{2}
$$

and $m_{\sigma} \ll H$. This field is called the curvaton field for reasons that will become clear shortly. The energy density of the curvaton field is negligible initially. During inflation, it fluctuates and obtains the primordial amplitude $\sigma_{*}=$ $\sigma_{0}+\delta \sigma(\mathbf{x})$, where $*$ denotes its value at the horizon-exit and after that the amplitude is approximately frozen. These perturbations are Gaussian for the potential (186) with the canonical kinetic term, but can be more complicated otherwise. Here, we study the simple Gaussian case. After inflation, these $\sigma$-modes remain frozen until the Hubble parameter drops below $m_{\sigma}$. Then the $\sigma$-field starts to oscillate around the bottom of the potential and behavior as matter. The Universe is still radiation dominated. The fraction of the matter energy density quickly grows, because the matter dilutes as $a^{-3}$ while radiation $a^{-4}$. The $\sigma$-field decays to radiation when it reaches its lifetime.

Another assumption of the curvaton model is that the primordial fluctuations in the inflaton field is much smaller than what is needed to achieve $\zeta \sim \mathcal{O}\left(10^{-5}\right)$, although their total energy density may still be the dominant one. So the primordial curvature perturbation is contributed by the fluctuations in the $\sigma$ field, hence the name curvaton field.

At the initial spatially flat slice $t_{0}$, we denote the radiation and curvaton density as $\rho_{r 0}$ and $\rho_{m 0}$, respectively, and the scale factor as $a_{0}$. Both components initially redshift as radiation. This lasts until the Hubble parameter reaches $m_{\sigma}$ at $t_{1}$. We denote the scale factor at $t_{1}$ as $a_{1}$. The Friedman equation at $t_{1}$ is

$$
3 M_{\mathrm{P}}^{2} m_{\sigma}^{2}=\left(\frac{a_{0}}{a_{1}}\right)^{4}\left(\rho_{r 0}+\rho_{m 0}\right) \approx\left(\frac{a_{0}}{a_{1}}\right)^{4} \rho_{r 0} .
$$

After this the curvaton starts to oscillate and behave as matter. Denote the decay rate of the curvaton as $\Gamma$. We use the sudden decay approximation and assume that they decay instantaneously at the epoch $H=\Gamma$, because a process that has the time scale $T$ falls into the Hubble expansion epoch with $H=1 / T$. We denote this instant as $t_{2}$. The Friedman equation at $t_{2}$ is

$$
3 M_{\mathrm{P}}^{2} \Gamma^{2}=\left(\frac{a_{0}}{a_{2}}\right)^{4} \rho_{r 0}+\left(\frac{a_{0}}{a_{1}}\right)^{4}\left(\frac{a_{1}}{a_{2}}\right)^{3} \rho_{m 0} .
$$

Because at $t_{2}$ the universe has the same Hubble parameter (hence the same energy density) everywhere, this is the final uniform density slice. After that, both components become radiation and the evolution everywhere is the same. So we want to work out the expansion e-folds $N$ from $t_{0}$ to $t_{2}$ as a function of the initial field value $\sigma$. From (188), we get

$$
e^{-4 N}+e^{-3 N} \alpha=\text { const. }
$$

where $\alpha \equiv\left(a_{0} / a_{1}\right)\left(\rho_{m 0} / \rho_{r 0}\right)$. From (187) we can solve for $a_{0} / a_{1}$ which is independent of $\sigma$ at the leading order, and from (186) we know that $\rho_{m 0}$ is proportional to $\sigma^{2}$. Therefore, $\alpha$ is proportional to $\sigma^{2}$. Also note that $r_{\rho} \equiv$ $\left(a_{2} / a_{0}\right) \alpha=e^{N} \alpha=\rho_{m} /\left.\rho_{r}\right|_{t_{2}}$ is the ratio of the energy density between the curvaton and the rest of the radiation at $t_{2}$. Using these simple facts, we can differentiate (189) with respect to $\sigma$ once and twice, and get

$$
f_{\mathrm{NL}}^{\mathrm{loc}}=\frac{5}{6} \frac{N_{\sigma \sigma}}{N_{\sigma}^{2}}=\frac{5}{3 r_{\rho}}-\frac{5\left(4+9 r_{\rho}\right)}{12\left(4+3 r_{\rho}\right)} .
$$

In terms of the definition $r \equiv 3 \rho_{m} /\left.\left(4 \rho_{r}+3 \rho_{m}\right)\right|_{t_{2}}=3 r_{\rho} /(4+$ $3 r_{\rho}$ ) often used in the literature,

$$
f_{\mathrm{NL}}^{\mathrm{loc}}=\frac{5}{4 r}-\frac{5}{3}-\frac{5 r}{6}
$$

So Large local non-Gaussianity arises if $r \ll 1$. Note that although (189) only depends on $\sigma$, this is a multifield model because the curvaton takes effects during the reheating. In some simple models in which the curvaton leads to nonadiabatic perturbations between dark matter and photons, $r$ is tightly constrained by observations [152].

The large local form has been studied most extensively in the past. Variety of possibilities exist. They all share the common feature that non-Gaussianities are generated by some massless isocurvaton fields which acquire the superhorizon evolution during the inflation. For example, in multifield slow-roll inflation a turning trajectory [153] can transfer non-Gaussianities from other directions to the inflaton direction [127-134]. But it is found to be very difficult to make non-Gaussianities large essentially because the very restrictive slow-roll conditions in all directions. In modulated reheating $[154,155]$ or preheating [156-161] scenarios, the role of isocurvatons are played by the massless fields which control the couplings during the reheating or preheating. Thus they create a large local non-Gaussianity in a similar fashion as the curvaton model. 
Local form is also found in different contexts, such as models with special types of massive gauge fields that acquire superhorizon evolution [162-164], some nonlocal theories of inflation $[165,166]$, and certain cyclic universe scenario as alternatives to inflation [167-171].

The current $\mathrm{CMB}$ constraint on the local bispectrum is $-10<f_{\mathrm{NL}}^{\text {local }}<74$ [1]. Current constraint from large scale structure gives $-29<f_{\mathrm{NL}}^{\text {local }}<70$ [172]. Variety of methods have been invented to measure the local and other different forms of non-Gaussianities [173-186].

\section{Summary and Discussions}

9.1. Summary. In this subsection, we summarize the main results of Sections 6-8. Non-Gaussianities, conceptually being the expectation values of perturbations in a timedependent background, are defined by the first-principle in-in formalism. Physically, having large primordial nonGaussianities means that there are large nonlinear interactions of some sort determined by certain dynamics during inflation. Measuring them tells us the nature of the dynamics.

(i) Equilateral Shape and Higher Derivative Kinetic Terms. In single field inflation, the long wavelength modes that already exited the horizon are frozen. They cannot have large interactions with short wavelength modes that are still within the horizon. When modes are well within the horizon, they oscillate and the contributions to non-Gaussianities average out. Therefore the only chance to have large interaction is when all modes have similar wavelengths and exit the horizon at about the same time. Theories with higher derivative kinetic terms provide such interaction terms. This is why the resulting bispectrum shape peaks at the equilateral limit in the momentum space. It drops to zero at the squeezed limit $k_{3} \ll k_{1}=k_{2}$ as $k_{3} / k_{1}$. It happens that, when these higher derivative terms become important enough so that the inflationary mechanism is no longer slow-roll, these large non-Gaussianities become observable. The forms of the bispectra are given in (121) and (122), and the shapes are plotted in Figures 3 and 4. The factorizable ansatz that is used to represent them in data analyses is given in (127) and plotted in Figure 5.

(ii) Sinusoidal Running and Sharp Feature. A sharp feature, in a potential or internal field space, introduces a sharp change in the slow-roll parameters, or the generalized slowvariation parameters. This can boost the magnitudes of time-derivatives of some parameters by several orders of magnitude while still keep the power spectrum viable. These time-derivatives act as couplings in the interaction terms. So they enhance the non-Gaussianities among the modes which are near the horizon-exit. How deep they affect the modes inside the horizon depends on how sharp the changes are.

The changes in these parameters can be roughly approximated as delta-functions in time. Correlation functions involve integrations of products of the slow-variation parameters and the mode functions. The latter contain oscillatory behavior $\sim e^{-i K \tau}$, where the comoving momentum $K$ is $k_{1}+$ $k_{2}+k_{3}$ for bispectra and $\tau$ is the conformal time. The deltafunction specifies a scale $\tau_{*}$. This is why after integration the bispectrum contains a sinusoidal factor $\sim \sin \left(K / k_{*}\right)$, where $k_{*}=-1 / \tau_{*}$ is the momentum of the mode that is near the horizon-exit at the time of the feature. So the most important property of this type of non-Gaussianity is this characteristic running. A numerical result of the running behavior is plotted in Figure 7. An ansatz is given in (137) and (139).

(iii) Resonant Running and Periodic Features. The periodic features do not have to be sharp. They introduce a small background oscillatory component in the slow-variation parameters. On the other hand, the mode functions are also oscillatory before they exit the horizon. Their frequencies are high when they lie deep inside the horizon and become lower as their wavelengths get stretched by the inflation. They are frozen after the wavelengths become comparable with the horizon size $H^{-1}$. This means that their frequencies continuously scan through the range from $M_{\mathrm{P}}$ (or some other large fundamental scale) to $H$. Therefore as long as the background oscillatory frequency $\omega$ satisfies $H<\omega<$ $M_{\mathrm{P}}$, at some point during the evolution the small oscillatory component in the slow-variation parameters will resonant with the oscillatory behavior of the mode functions, and cause a large constructive contribution to the integration.

The periodicity of the features leads to a periodicscale-invariance in density perturbations. Namely, they are scale invariant if we rescale all momenta by a discrete efold $2 \pi n H / \omega$, where $n$ is an integer. This is why the most important feature of this non-Gaussianity is a running behavior $\sim \sin (C \ln K+$ phase $)$, where $C=\omega / H$. This leads to the ansatz (148). The full expression is given in (150) and plotted in Figure 8. A numerical result is plotted in Figure 9.

(iv) Folded Shape and Non-Bunch-Davies Vacuum. The usual mode function of the Bunch-Davies vacuum has the positive energy mode $\sim e^{-i k \tau}$. Now we consider a non-Bunch-Davies vacuum by adding a small component of negative energy mode $\sim e^{i k \tau}$. The three-point function involves an integration of the product of three mode functions with momentum $k_{1}$, $k_{2}$ and $k_{3}$. So the leading correction to the Bunch-Davies results is to replace one of the $k_{i}$ s with $-k_{i}$. The usual $K=$ $k_{1}+k_{2}+k_{3}$ in $e^{-i K \tau}$ becomes $\widetilde{K}=k_{1}+k_{2}-k_{3}$, and its cyclic. This effect is most important if factors of $\widetilde{K}$ appear in the denominators after the $\tau$-integration. Hence, the most important feature of this type of modification is to enhance the non-Gaussianity in the folded triangle limit. An example of these bispectra is given in (154) and plotted in Figure 10. Ansatz that partially capture this feature are given in (155) and (156), and plotted in Figure 11.

(v) Intermediate Shapes and Massive Isocurvatons. All mechanisms discussed so for single field inflation apply to multifield inflation. We now consider new effects caused by introducing more fields to inflation models. These extra fields are called isocurvatons. 
Since light fields typically acquire a mass of order $H$, the Hubble parameter, we first consider the quasisingle field inflation models where there is one massless inflaton while the isocurvatons have mass of order $H$ instead of massless.

Unlike multifield slow-roll inflation, where each flat direction only has small nonlinear terms in order to satisfy the slow-roll conditions, massive directions are not inflationary direction and are free to have large nonlinear selfinteractions. These nonlinear interactions can be transferred to the curvature mode through couplings and source the large non-Gaussianity.

The massive isocurvaton eventually decays after horizon exit simply because they are diluted by the expansion. How fast it decays depends on its mass. If the mass is heavier, $m>$ $\sqrt{2} H$, it decays faster. So the interactions can only happen when all modes are all closer to the horizon exit. This is closer to the case of the equilateral shape that we encountered above, and results in bispectra with quasiequilateral shapes. If the mass is lighter, $m<\sqrt{2} \mathrm{H}$, it decays slower. More non-Gaussianity is generated in the superhorizon scales. This is closer to the case of the local shape that we will come to below, and results in bispectra with quasilocal shapes. Overall, at the squeezed limit $k_{3} \ll k_{1}=k_{2}$, the bispectrum shapes behave as $\left(k_{3} / k_{1}\right)^{1 / 2-v}$, where $v$ goes from 0 to $3 / 2$ (corresponding to $m$ from $3 H / 2$ to 0 ) in the example we studied. In particular, if we take the massless limit while keeping the cubic self-interactions of isocurvaton large, we get a large bispectrum that has the same squeezed limit shape as the local one. Therefore, we have a one-parameter family of shapes that lie between the local and equilateral shape.

The numerical results of these shapes are presented in Figure 14. A simple ansatz is given in (174) that represents this family of shapes quite well, and is plotted in Figure 15.

(vi) Local Shape and Massless Isocurvatons. The fluctuation amplitudes of massless scalars do not decay after the horizon exit, and therefore this opens up a multifield space for the superhorizon evolution. For superhorizon modes, we can use the separate universe picture and study the classical behavior of different patches of universe. These patches are separated by horizons and evolve independently of each other. So the evolution is local in space.

Non-Gaussianities are generated when this multifield evolution is nonlinear, and any nonlinearity arising in the separate universe picture should also be local in space. A locality in position space translates to a nonlocality in momentum space. This is why the resulted local shape bispectrum peaks at the squeezed limit. The behavior is $\left(k_{3} / k_{1}\right)^{-1}$ for $k_{3} \ll k_{1}=k_{2}$. This bispectrum is given in (184) and the shape is plotted in Figure 16.

In all cases, the power spectra are either approximately scale-invariant so indistinguishable from the simplest slowroll models, or modified with features that can be made small enough to satisfy the current observational constraints.
Large bispectra generically implies large trispectra, that is, the four-point correlation functions. But trispectra contain more information and can be large even if bispectra are small. Experimentally, trispectra are more difficult to detect, but contain much more shape configurations. Each category above should have interesting extensions to trispectra. See [187-193] for the equilateral case and [194-199] for the local case.

It is certain that this list will grow in future works, providing more refined and diverse connections between theories and experiments.

9.2. A Consistency Condition. As we have seen, in single field inflation, the mode that has exited the horizon is frozen. This is characterized by a constant $\zeta$ over a horizon size patch. The physical meaning of the constant $\zeta$ is a small rescaling of the scale factor. This is the only effect that the superhorizon mode has on modes with much shorter wavelength. This fact is used by Maldacena to derive a consistency condition [47] for the three-point correlation functions in the squeezed limit for single field inflation.

(i) Consistency Condition. In the squeezed limit $k_{3} \ll k_{1}=$ $k_{2}, k_{3}$ is the superhorizon mode that exited the horizon and acts as a zero-mode modulation to the two remaining modes. The correlation $\left\langle\zeta_{k_{1}} \zeta_{k_{2}} \zeta_{k_{3}}\right\rangle$ is an average of the following quantity

$$
\left\langle\zeta_{k_{1}} \zeta_{k_{2}}\right\rangle_{\zeta_{k_{3}}} \zeta_{k_{3}}
$$

over different $\zeta_{k_{3}}$. We will shift the average $\left\langle\zeta_{k_{3}}\right\rangle$ to zero by definition. Here the two-point average $\left\langle\zeta_{k_{1}} \zeta_{k_{2}}\right\rangle_{\zeta_{k_{3}}}$ is evaluated with different local scalings determined by the shift $\zeta_{k_{3}}$. If the two-point function is exactly scale-invariant, $\left\langle\zeta_{k_{1}} \zeta_{k_{2}}\right\rangle_{\zeta_{k_{3}}}$ is just a constant. So the $3 \mathrm{pt}$ vanishes because $\left\langle\zeta_{k_{3}}\right\rangle=0$. The nonzero contribution comes from the breaking of the scale invariance. To see this, we expand the two-point average in terms of a long wavelength mode $\zeta_{k_{4}}$ near the scale $\left\langle\zeta_{k_{4}}\right\rangle=0$,

$$
\begin{aligned}
\left\langle\zeta_{k_{1}} \zeta_{k_{2}}\right\rangle_{\zeta_{4}}= & \left\langle\zeta_{k_{1}} \zeta_{k_{2}}\right\rangle_{0}+\left.\frac{d\left\langle\zeta_{k_{1}} \zeta_{k_{2}}\right\rangle}{d \zeta_{k_{4}}}\right|_{0} \zeta_{k_{4}} \\
& +\left.\frac{1}{2} \frac{d^{2}\left\langle\zeta_{k_{1}} \zeta_{k_{2}}\right\rangle}{d \zeta_{k_{4}}^{2}}\right|_{0} \zeta_{k_{4}}^{2}+\cdots
\end{aligned}
$$

Multiply this with $\zeta_{k_{3}}$ and average over $\zeta_{k_{3}}$. The first term contributes zero since this is the scale-invariant component. The second term gives

$$
\left.\frac{d\left\langle\zeta_{k_{1}} \zeta_{k_{2}}\right\rangle}{d \zeta_{k_{4}}}\right|_{0}\left\langle\zeta_{k_{3}} \zeta_{k_{4}}\right\rangle
$$

To get nonzero average, $\mathbf{k}_{3}+\mathbf{k}_{4}=0$ is needed. Using the relation $d \zeta_{k_{4}}=-d \ln k$, we get

$$
\left\langle\zeta_{k_{1}} \zeta_{k_{2}} \zeta_{k_{3}}\right\rangle \longrightarrow-\left.\frac{d\left\langle\zeta_{k_{1}} \zeta_{k_{2}}\right\rangle}{d \ln k}\right|_{0}\left\langle\zeta_{k_{3}} \zeta_{k_{3}}\right\rangle
$$


The higher-order terms in (193) give

$$
\left.\frac{1}{2} \frac{d^{2}\left\langle\zeta_{k_{1}} \zeta_{k_{2}}\right\rangle}{(d \ln k)^{2}}\right|_{0}\left\langle\zeta_{k_{3}} \zeta_{k_{4}}^{2}\right\rangle-\left.\frac{1}{6} \frac{d^{3}\left\langle\zeta_{k_{1}} \zeta_{k_{2}}\right\rangle}{(d \ln k)^{3}}\right|_{0}\left\langle\zeta_{k_{3}} \zeta_{k_{4}}^{3}\right\rangle+\cdots,
$$

where $\mathbf{k}_{3}+2 \mathbf{k}_{4}=0, \mathbf{k}_{3}+3 \mathbf{k}_{4}=0$, and so on have to be satisfied, respectively, for each term to get nonzero average. If we only consider the tree-level three-point function, these higher-order terms can be truncated since they involve more factors of $P_{\zeta}$ and should be related to the loop diagram contributions to $\left\langle\zeta_{k_{1}} \zeta_{k_{2}} \zeta_{k_{3}}\right\rangle$. The tree diagram is $\mathcal{O}\left(P_{\zeta}^{2}\right)$.

To connect the averages we used here with the correlation functions that we defined in previous sections, we need to restore the phase factors. Here the two-point average $\left\langle\zeta_{k_{1}} \zeta_{k_{2}}\right\rangle_{\text {here }}$ is performed with the special point $k_{1}=k_{2}$ in the phase space. To connect this with the previous definition of $\left\langle\zeta_{k_{1}} \zeta_{k_{2}}\right\rangle$, we need to include the phase space in the neighborhood. Namely, $\left\langle\zeta_{k_{1}} \zeta_{k_{2}}\right\rangle=\left\langle\zeta_{k_{1}} \zeta_{k_{2}}\right\rangle_{\text {here }}(2 \pi)^{3} \delta^{3}\left(\mathbf{k}_{1}+\right.$ $\mathbf{k}_{2}$ ), so from the definition (26) we have $\left\langle\zeta_{k_{1}} \zeta_{k_{2}}\right\rangle_{\text {here }}=$ $(2 \pi)^{2} P_{\zeta}\left(k_{1}\right) / 2 k_{1}^{3}$. Similarly, $\left\langle\zeta^{3}\right\rangle=\left\langle\zeta^{3}\right\rangle_{\text {here }}(2 \pi)^{3} \delta^{3}\left(\sum \mathbf{k}_{i}\right)$. With the usual definition of the spectrum index $n_{s}-1 \equiv$ $d \ln P_{\zeta} / d \ln k$, from (195), we get the following consistency condition [47]:

$$
\left\langle\zeta_{k_{1}} \zeta_{k_{2}} \zeta_{k_{3}}\right\rangle \longrightarrow-\left(n_{s}-1\right) \frac{1}{4 k_{1}^{3} k_{3}^{3}} P_{\zeta}\left(k_{1}\right) P_{\zeta}\left(k_{3}\right)(2 \pi)^{7} \delta^{3}\left(\sum_{i} \mathbf{k}_{i}\right) .
$$

Although originally derived for slow-roll inflation, the only assumption is the single field. So this applies to any single field inflation models and has important physical implications that we discuss shortly [200]. Note that the derivation of this relation (197) does not rely on the smallness of the slow-variation parameters either. For the general single field inflation models that we studied in Section 6.1, at tree level this has been checked with explicit results to three different orders $[50,201]$ including the slowroll limit [47]. For resonance models, this is checked to the leading order [111].

There are three types of interesting corrections to the condition (197).

Firstly, as mentioned, the right-hand side of (197) should receive corrections from loop contributions. These loop contributions are associated with higher derivatives of the two-point function. The terms (196), together with (195), provide the corresponding consistency conditions at different orders of $P_{\zeta}$. Note that for such orders, the correlation functions such as $\left\langle\zeta_{k_{1}} \zeta_{k_{2}}\right\rangle$ on the right-hand side should also include loop corrections.

Secondly, when we assume that the only effect of the frozen superhorizon mode on the much shorter scale is a constant background rescaling, we assume that there is no interaction when these modes are all within the horizon (I would like to thank Yi Wang for helpful discussions on this point). However, large subhorizon interaction is possible in some cases, such as in Sections 6.3 and 6.4. Such interactions disappear below a new length scale at subhorizon, only then the above assumption becomes valid. For example in the resonance model, for $H / \omega<k_{3} / k_{1} \ll 1$, the modes $\mathbf{k}_{1}$, $\mathbf{k}_{2}$ and $\mathbf{k}_{3}$ are guaranteed to resonant with the oscillatory background at some point when all of them are within the horizon. Only if $k_{3} / k_{1} \ll H / \omega$, such resonance will not happen. This is why the consistency condition is satisfied only in the very squeezed limit $\left(k_{3} \ll k_{1} / C\right.$ with $C=\omega / H \gg$ $1)$. For the squeezed region $k_{1} / C<k_{3} \ll k_{1}$, the left-hand side of the condition is larger than the right-hand side by a factor of $C k_{3} / 2 k_{1}$. For the non-Bunch-Davies vacuum case, a similar scale is determined by the UV cutoff $\tau_{c}$, from which the non-Bunch-Davies vacuum starts to take effect.

Thirdly, even after the long wavelength mode exits the horizon, as long as $k_{3} / k_{1}$ is not infinitely small, there is still dependence of the two-point function on the derivative of this long wavelength mode, in addition to the overall constant shift. This introduces a different type of finite $k_{3} / k_{1}$ corrections. They start from the second-order in $k_{3} / k_{1}$, because the first-order corresponds to the first spatial derivative of the long wavelength mode and should vanish due to isotropy [200]. These corrections will be amplified by the associated amplitude $f_{\mathrm{NL}}^{\text {non-loc }}$, and give an additive correction $f_{\mathrm{NL}}^{\text {non-loc }}\left(k_{3} / k_{1}\right)^{2}$ to the $n_{s}-1$ on the right-hand side of the condition. For a large $f_{\mathrm{NL}}^{\text {non-loc }}$, therefore, the condition needs to be satisfied in a very squeezed limit. The equilateral bispectra (121) and (122) are this type of examples.

The consistency condition (197) can be straightforwardly generalized to higher-order correlation functions $[189,194]$. We emphasize that this condition only applies to single field inflation models. For inflation models involving more than one field, as we have seen, non-Gaussianities can be transferred from the isocurvature directions which do not respect this relation.

(ii) Physical Implication. Besides providing consistency checks for analytical computations, the condition also has interesting physical implications. In the following, we discuss the scale invariant cases [200], as well as the feature cases and loop corrections, ending with some cautionary remarks.

This consistency relation implies that the tree-level bispectrum in the squeezed limit is determined by the power spectrum and spectral index. We distinguish the following two cases. For the scale-invariant case, $n_{s}-1$ is of order $\mathcal{O}(\epsilon)$ and the right-hand side of (197) takes the local form. Indeed, as we have seen, for the single field inflation models where the non-Gaussianities are large, they take the equilateral forms which vanish in the infinitely squeezed limit. For the non-scale-invariant case, especially the highly oscillatory case such as the resonance model, the power spectrum can be highly oscillatory and $n_{s}-1$ becomes large. This can still be consistent with observations since the large $n_{s}-1$ is also highly oscillatory and therefore may escape a detection so far. But such a running non-Gaussianity is orthogonal to the scale-invariant forms.

For the loop diagrams, in the scale-invariant case, these terms are suppressed by higher-orders of slow-variation parameters from, for example, $d^{2}\left\langle\zeta_{k_{1}} \zeta_{k_{2}}\right\rangle /(d \ln k)^{2}$, and 

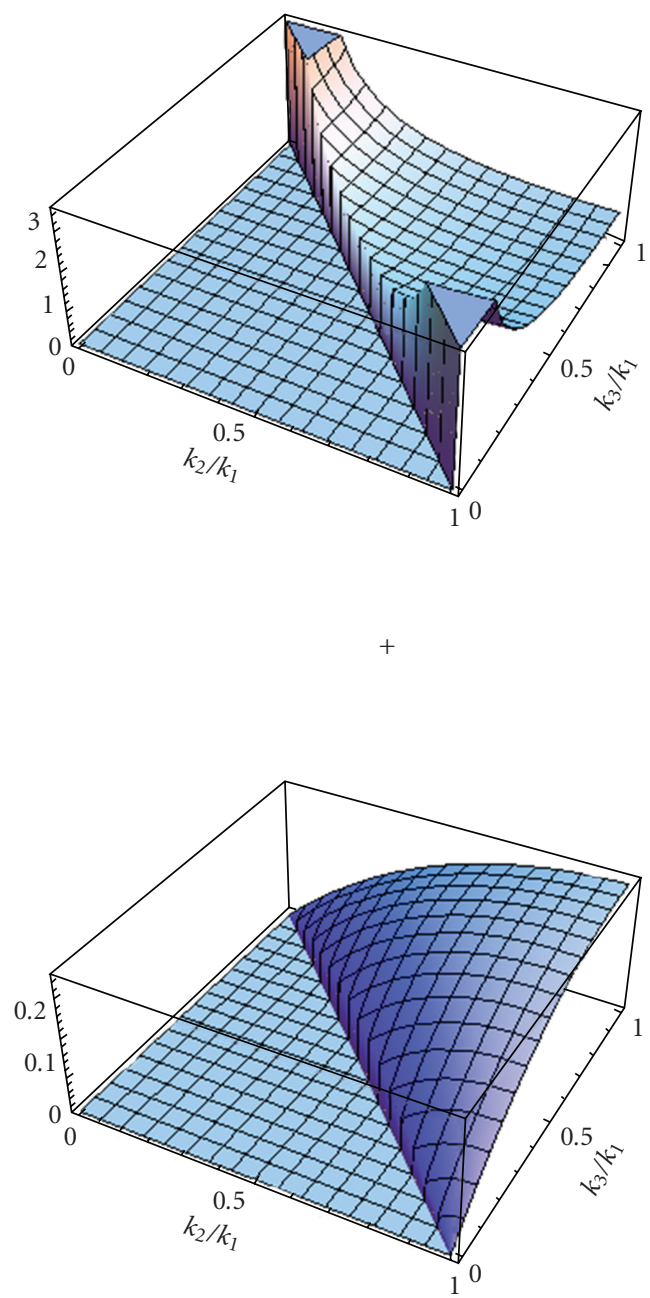

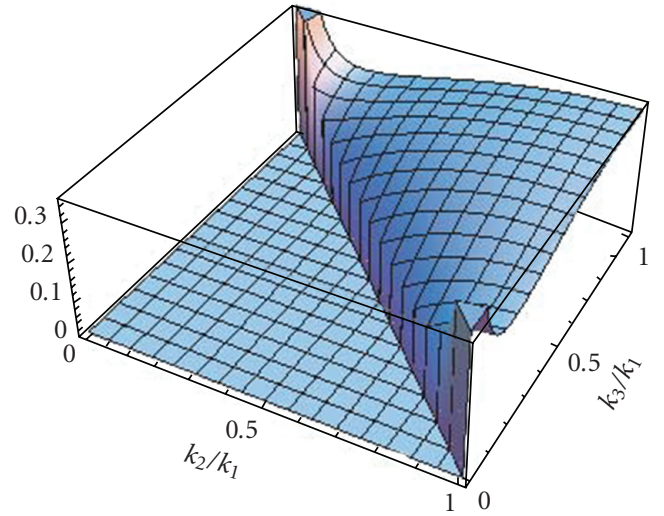

FIGURE 17: A mixing of the equilateral (Figure 4) and local shape (Figure 16).

higher-orders of $\zeta$ from, for example, $\left\langle\zeta_{k_{3}}^{3}\right\rangle$; in the nonscale-invariant example, the extra terms are still highly oscillatory.

In summary, a detection of an approximately scaleinvariant local non-Gaussianity in the infinitely squeezed triangle limit with $f_{\mathrm{NL}}^{\text {loc }}>\mathcal{O}(\epsilon)$ can rule out all single field inflation models.

In experiments, however, the triangle cannot be perfectly squeezed. So it is an important question how squeezed it should be to achieve the above goal. For example, in the third type of corrections we discussed previously in this subsection, we need $f_{\mathrm{NL}}^{\text {non-loc }}\left(k_{3} / k_{1}\right)^{2}$ to be smaller than $n_{s}-1$ for the consistency condition to hold, so that the contaminations from whatever nonlocal $f_{\mathrm{NL}}$ is small. Assuming the primordial local form is practically detectable only if $f_{\mathrm{NL}}^{\text {loc }}>\mathcal{O}(1)$, we at least need $f_{\mathrm{NL}}^{\text {non-loc }}\left(k_{3} / k_{1}\right)^{2}<\mathcal{O}(1)$. For the class of the general single field models we studied in Section 6, if the other forms of non-Gaussianities, such as the equilateral one, can be constrained below $f_{\mathrm{NL}}^{\text {non-loc }} \sim \mathcal{O}(10)$, a squeezed configuration with $k_{3} / k_{1}<0.1$ will be enough for our purpose. However, a completely model-independent statement is much trickier, because there may be bispectra with very large amplitude but orthogonal to any known bispectra that have been constrained experimentally. Besides that, in the second type of corrections, large finite- $k_{3} / k_{1}$ corrections can also arise due to subhorizon interactions. Therefore, as a cautionary remark, if we would like to rule out all single field inflation models in a rigorous modelindependent fashion with a detection of scale-invariant local non-Gaussianity, we have to keep in mind the caveat that there may be single field models which only respect the consistency condition in a very squeezed region beyond the experimental reach.

9.3. Superpositions. Different shapes and runnings of nonGaussianities can be superimposed in inflation models. For example, consider the following.

(i) Mixing Shapes. It is possible that different nonGaussianity generation mechanisms are from different components in a model, or at different stages during inflation. So two or more different shapes can get mixed, and the final shape can be rather different. For example, in Figure 17, we plot a mixed shape between the local and equilateral shape. 

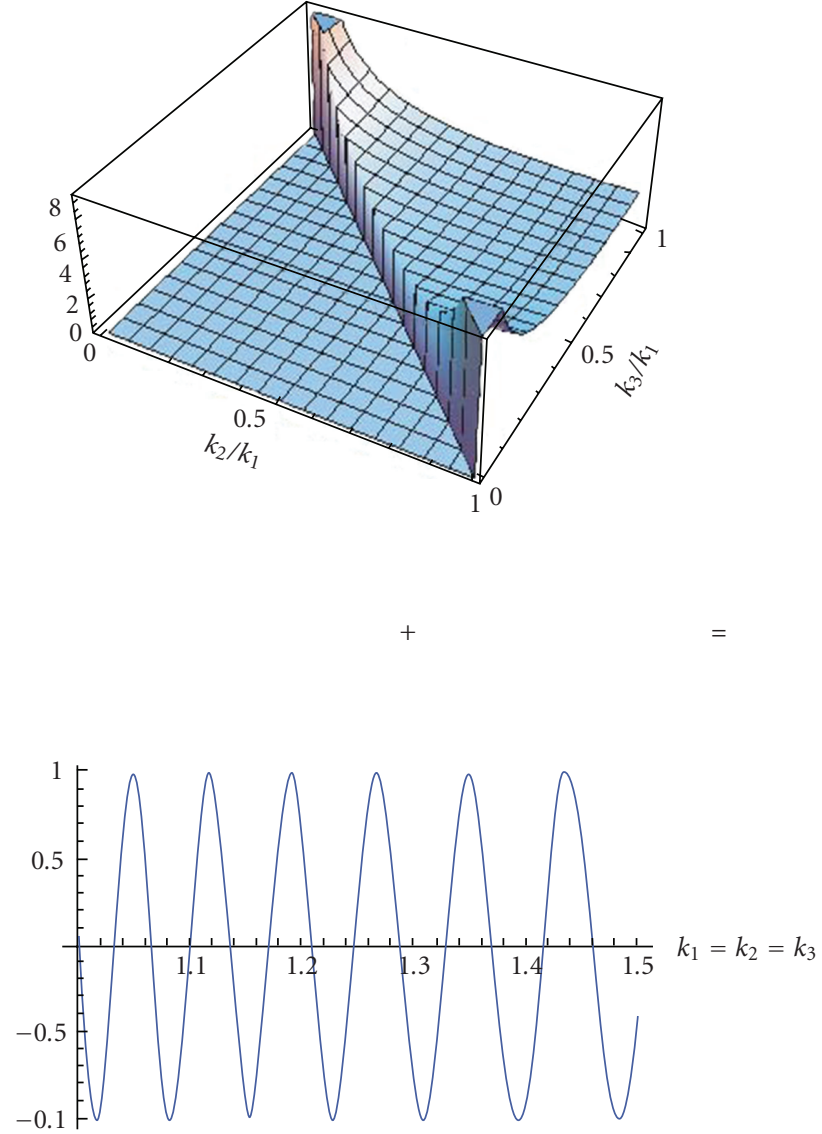

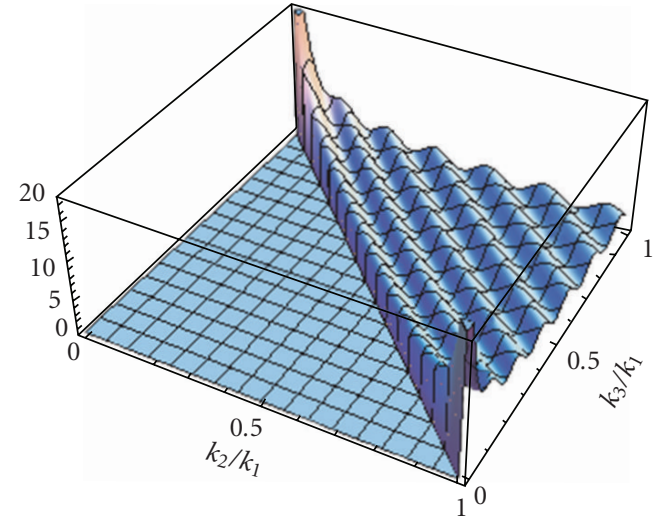

FIgURE 18: A mixing of an intermediate shape $[\nu=7 / 6$ in (174) $]$ and a resonant running (148).
Notice that this is different from the intermediate shapes, since obviously the squeezed limit is always dominated by the local form. Examples of such models are discussed in [202].

(ii) Mixing Shape and Running. The shapes can also be mixed with runnings. Same as the power spectrum, the nonGaussianities generically have some mild scale dependence. But a more dramatic case is the superposition with a strong running, such as the sinusoidal or resonant running. For example, an inflaton passing through features frequently and turning constantly at the same time on a potential landscape can generate a bispectrum which is a superposition of the resonant running and intermediate shape, as we illustrate in Figure 18. Clearly, these two signals are orthogonal to each other very well, and have to be picked up separately through different methods in data analyses.

(iii) Orthogonalization. If a non-Gaussianity is the linear superposition of several base components, one can generally perform a change of bases to make the new bases orthogonalized. For example, as we have seen in Section 6.1, the leading large bispectrum has two components, $S_{\lambda}$ and $S_{c}$. The two shapes are very similar, and represented by the equilateral ansatz in data analyses. However since they do have small difference, one can subtract their similarities and get a new orthogonalized base component [175]. The orthogonalization is defined by the shape correlator such as (106). Using this definition, the new bases can be chosen as

$$
S_{1} \approx S_{\lambda}+0.22 S_{c}
$$

and $S_{2}=S_{c}$. [Note that the $S_{\lambda}$ and $S_{c}$ used here do not include the prefactors $\left(1 / c_{s}^{2}-1-2 \lambda / \Sigma\right)$ and $\left(1 / c_{s}^{2}-1\right)$ in $(121)$ and (122)]. Their shapes are shown in Figure 19. Notice that $S_{1}$ is half positive and half negative. Because $S_{1}$ is not of the simplest factorizable type, the following simple ansatz has been proposed to represent $S_{1}$ in data analyses [175],

$$
S_{\text {ansatz }}^{\text {orth }}=-18\left(\frac{k_{1}^{2}}{k_{2} k_{3}}+2 \text { perm. }\right)+18\left(\frac{k_{1}}{k_{2}}+5 \text { perm. }\right)-48 .
$$

We plot the shape of this ansatz in Figure 20. The current $\mathrm{CMB}$ constraint on this orthogonal ansatz is $-410<f_{\mathrm{NL}}^{\text {orth }}<$ $6[1]$.

For known examples of general single field inflation, such as the DBI and k-inflation, we generically get equilateral shapes. This is also clear from their physical origin that we have emphasized. The orthogonal shape relies on a delicate cancellation between the two generic shapes. In principle, one can do this since the required parameter space is allowed in our effective field theory of general single field inflation in 

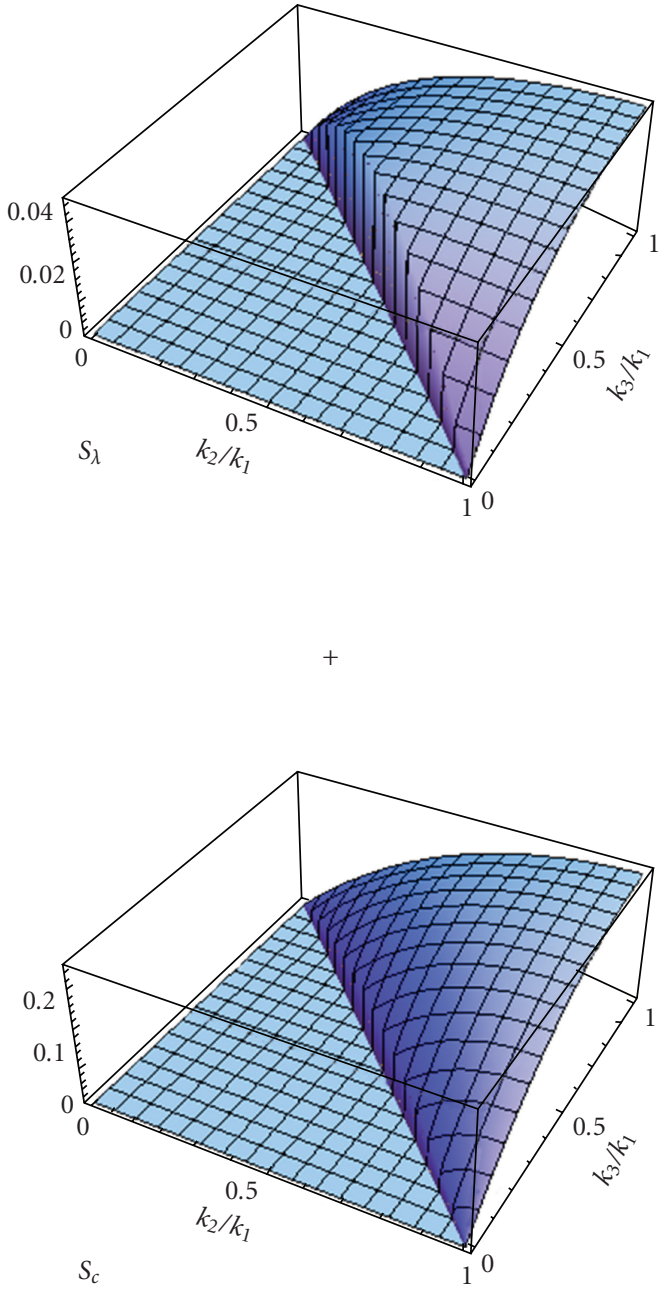

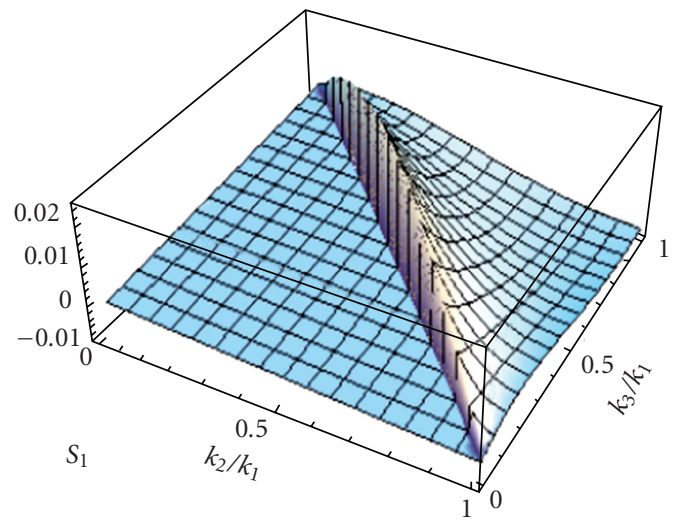

Change of bases

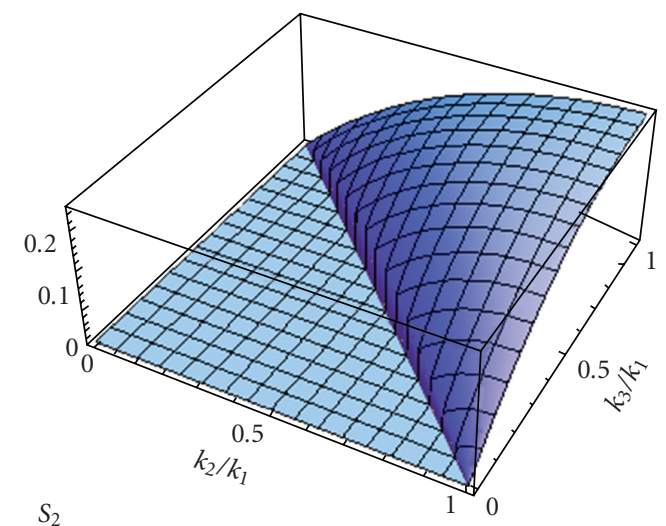

$S_{2}$

Figure 19: Orthogonalization of two shapes in Section 6.1 (Figures 3 and 4) through a change of bases, $c_{\lambda} S_{\lambda}+c_{c} S_{c}=c_{1} S_{1}+c_{2} S_{2}$.

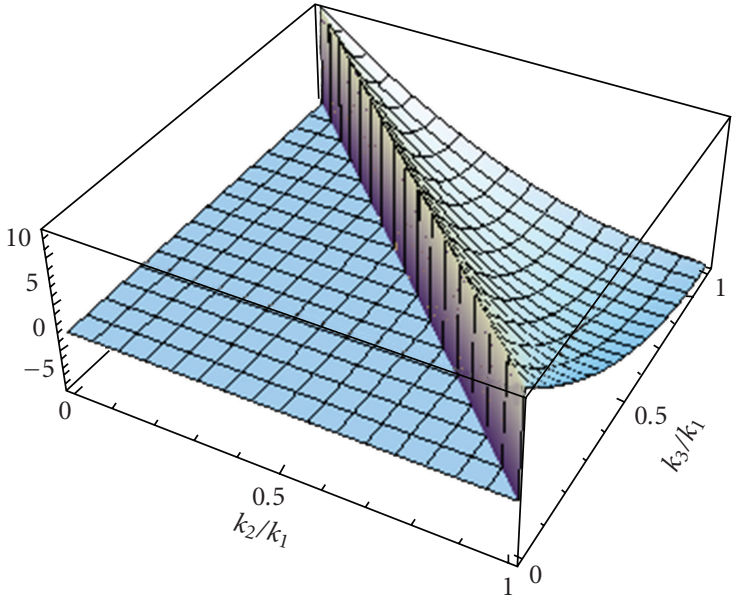

FIGURE 20: An ansatz $-S_{\text {ansatz }}^{\text {orth }}$ in (199) for the orthogonal shape $S_{1}$ in (198). Note we added a minus sign in this plot.

Section 6.1, and this may provide guidance to future model building. For example, one may fine-tune the parameters in the k-inflation models $[93,94]$. Therefore, unlike the previous cases, the direct motivation here is more oriented to data analyses. The advantage of this operation is that it makes full use of data, which impose constraints on both components. In addition, as a bonus, the ansatz for the equilateral (127), folded (155) and orthogonal (199) shapes are not linearly independent. As we can see, they all happen to be the equilateral ansatz shifted by a constant shape ansatz ( $S=$ const.) [65]. Constraining two orthogonal bases provide efficient constraints on all three of them.

Let us do a more data-analysis-oriented exercise. We would like to construct an ansatz that is orthogonal to both local and equilateral ansatz, since both were well constrained by data. (Note that $S_{\text {ansatz }}^{\text {orth }}$ in (199) is not quite orthogonal to the local ansatz, with a correlation $\sim-0.48$.) To do this we start with a trial shape $S_{\text {trial }}$, and demand the new orthogonal shape

$$
S_{\text {ansatz }}^{\text {orth }}=S_{\text {trial }}+c_{1} S^{\text {loc }}+c_{2} S_{\text {ansatz }}^{\text {eq }}
$$

be orthogonal to both the local and equilateral ansatz,

$$
S_{\text {ansatz }}^{\text {orth } 2} \cdot S^{\text {loc }}=0, \quad S_{\text {ansatz }}^{\text {orth } 2} \cdot S_{\text {ansatz }}^{\text {eq }}=0 .
$$




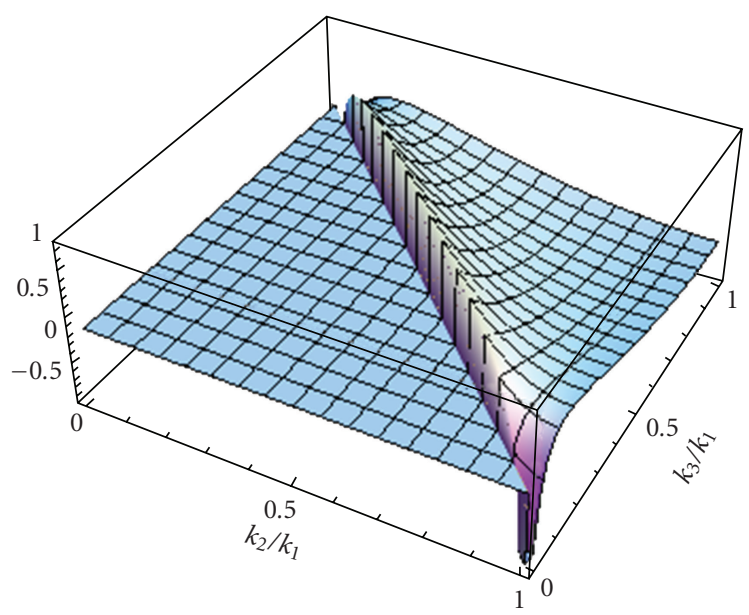

Figure 21: Another factorizable orthogonal ansatz (202).

The simplest factorizable trial shapes can be either the constant shape or the local-like shape $k_{1} / k_{2}+5$ perm., and both give the same result. Let us use the constant shape $S_{\text {trial }}=1$ as an example. Solving the conditions (201) gives $c_{1}=-0.0953$ and $c_{2}=-0.204$. So the new orthogonal ansatz is

$S_{\text {ansatz }}^{\text {orth } 2}=1.19\left(\frac{k_{1}^{2}}{k_{2} k_{3}}+2\right.$ perm. $)-1.22\left(\frac{k_{1}}{k_{2}}+5\right.$ perm. $)+3.44$.

The numerical details may change slightly depending on the detailed definition and computation of the inner product (105). The shape is plotted in Figure 21. It is somewhat exotic but the ansatz is simple. By construction, this ansatz is much more orthogonal to the local form (with correlation $\sim 0)$ than the $S_{\text {ansatz }}^{\text {orth }}$ currently used in $[1,175]$. It also happens to have reasonably large correlation $(\sim 0.86)$ with the orthogonal shape in single field inflation (the $S_{1}$ shown in Figure 19), similar to that $(\sim-0.91)$ between $S_{1}$ and $S_{\text {ansatz }}^{\text {orth }}$. Obviously, other choices of trial shapes can result in more exotic orthogonal shapes.

One can perform a similar orthogonalization for the two shapes in (94), now they are both local to start with. More generally, if a non-Gaussianity has more base components, we can orthonormalize all of them one by one, in the sense of the Gram-Schmidt process.

9.4. Conclusion. The field of primordial non-Gaussianity is growing rapidly in recent years, with simultaneous progress from the experimental results, data analyses methods, nonlinear cosmology theories, physical model buildings, computational techniques, and theoretical formalisms. The progress that we have seen so far is no doubt just a beginning.

In this paper, we have studied the primordial nonGaussianities coming from the inflation models, especially various mechanisms that can produce observable large nonGaussianities with viable power spectra. We emphasized the fingerprints that different underlying physics leave on nonGaussian profiles, which break the degeneracy of model building. We described the physical pictures and presented their effective Lagrangians to the extent that they can be recognized when encountered in the inflation model building in a more fundamental theory. We also derived the resulting bispectra and represented them in terms of simple ansatz to the extent that they can be useful to data analyses. With the current rapid progress, we anticipate much more future developments along these lines through refinements and discoveries in both theories and experiments.

The standard model of cosmology - the Big Bang theory with $\Lambda \mathrm{CDM}$ - is now established better than ever, with the precision data coming from the cosmic microwave background and large scale structures. New data will continue to flow from many ongoing and forthcoming experiments. Although Nature does not seem to be obligated to provide us any more information beyond the standard model, exciting possibilities exist that would help us to understand the origin of the Big Bang. These include the more detailed deviations from the scale-invariance of the power spectrum, the primordial gravitational waves that we may detect from the $\mathrm{CMB}$ polarization, the isocurvature perturbations between matter and radiation, and the primordial non-Gaussianities. Without these types of data, the number of theoretical models with degenerate observational consequences proliferate with time and it will be hard to understand the microscopic nature of the inflation beyond our current knowledge, as well as to distinguish inflation from other possible alternatives. As we have reviewed, primordial non-Gaussianity-the collider in the very early universe-is one of the few hopes. It is becoming a target of many modern experiments. We do not know which cards Nature is hiding from us, but we are hoping and preparing for the best.

\section{Acknowledgments}

The author would like to thank Rachel Bean, Richard Easther, Girma Hailu, Bin Hu, Min-xin Huang, Shamit Kachru, Eugene Lim, Hiranya Peiris, Sash Sarangi, Gary Shiu, Henry Tye, Yi Wang, and Jiajun Xu for valuable collaborations and sharing their insights on the works reviewed here. The author would also like to thank James Fergusson, Michele Liguori, David Lyth, David Seery, Paul Shellard, and David Wands for very helpful discussions. The author was supported by the Stephen Hawking advanced fellowship.

\section{References}

[1] E. Komatsu, et al., "Seven-Year Wilkinson Microwave Anisotropy Probe (WMAP) Observations: CosmologicalInterpretation," http://arxiv.org/abs/1001.4538.

[2] B. Gold, et al., "Seven-Year Wilkinson Microwave Anisotropy Probe (WMAP) Observations: GalacticForeground Emission," http://arxiv.org/abs/1001.4555.

[3] D. Larson, et al., "Seven-Year Wilkinson Microwave Anisotropy Probe (WMAP) Observations: PowerSpectra and WMAP-Derived Parameters," http://arxiv.org/abs/1001 .4635 . 
[4] J. L. Weiland, et al., "Seven-Year Wilkinson Microwave Anisotropy Probe (WMAP) Observations:Planets and Celestial Calibration Sources," http://arxiv.org/abs/1001.4731.

[5] N. Jarosik, et al., "Seven-Year Wilkinson Microwave Anisotropy Probe (WMAP) Observations: SkyMaps, Systematic Errors, and Basic Results," http://arxiv.org/abs/ 1001.4744.

[6] C. L. Bennett, et al., "Seven-Year Wilkinson Micro-wave Anisotropy Probe (WMAP) Observations: AreThere Cosmic Microwave Background Anomalies?” http://arxiv.org/ abs/1001.4758.

[7] A. H. Guth, "Inflationary universe: a possible solution to the horizon and flatness problems," Physical Review D, vol. 23, no. 2, pp. 347-356, 1981.

[8] A. D. Linde, "A new inflationary universe scenario: a possible solution of the horizon, flatness, homogeneity, isotropy and primordial monopole problems," Physics Letters B, vol. 108, no. 6, pp. 389-393, 1982.

[9] A. Albrecht and P. J. Steinhardt, "Cosmology for grand unified theories with radiatively induced symmetry breaking," Physical Review Letters, vol. 48, no. 17, pp. 1220-1223, 1982.

[10] V. F. Mukhanov and G. V. Chibisov, "Quantum fluctuation and nonsingular Universe,” JETP Letters, vol. 33, pp. 532-535, 1981 (Russian).

[11] S. W. Hawking, "The development of irregularities in a single bubble inflationary universe," Physics Letters B, vol. 115, no. 4, pp. 295-297, 1982.

[12] A. A. Starobinsky, "Dynamics of phase transition in the new inflationary universe scenario and generation of perturbations," Physics Letters B, vol. 117, no. 3-4, pp. 175-178, 1982.

[13] A. H. Guth and S.-Y. Pi, "Fluctuations in the new inflationary universe," Physical Review Letters, vol. 49, no. 15, pp. 1110 1113, 1982.

[14] J. M. Bardeen, P. J. Steinhardt, and M. S. Turner, "Spontaneous creation of almost scale-free density perturbations in an inflationary universe," Physical Review D, vol. 28, no. 4, pp. 679-693, 1983.

[15] E. W. Kolb and M. S. Turner, The Early Universe, AddisonWesley, Redwood city, Calif, USA, 1988.

[16] A. Linde, "Axions in inflationary cosmology," Physics Letters $B$, vol. 259, no. 1-2, pp. 38-47, 1991.

[17] A. R. Liddle and D. H. Lyth, Cosmological Inflation and LargeScale Structure, Cambridge University Press, Cambridge, UK, 2000.

[18] S. Dodelson, Modern Cosmology, Academic Press, Amsterdam, The Netherlands, 2003.

[19] V. Mukhanov, Physical Foundations of Cosmology, Cambridge University Press, Cambridge, UK, 2005.

[20] S. Weinberg, Cosmology, Oxford University Press, Oxford, UK, 2008.

[21] D. Wands, “Multiple field inflation," Lecture Notes in Physics, vol. 738, pp. 275-304, 2008.

[22] K. A. Malik and D. Wands, "Cosmological perturbations," Physics Reports, vol. 475, no. 1-4, pp. 1-51, 2009.

[23] W. H. Kinney, "TASI lectures on inflation," http://arxiv.org/ abs/0902.1529.

[24] D. Baumann, “TASI Lectures on Inflation," http://arxiv.org/ abs/0907.5424.

[25] D. Langlois, "Lectures on inflation and cosmological perturbations," http://arxiv.org/abs/1001.5259.

[26] D. H. Lyth and A. Riotto, "Particle physics models of inflation and the cosmological density perturbation," Physics Report, vol. 314, no. 1-2, pp. 1-146, 1999.
[27] F. Quevedo, "Lectures on string/brane cosmology," Classical and Quantum Gravity, vol. 19, no. 22, pp. 5721-5779, 2002.

[28] S.-H. H. Tye, "Brane inflation: string theory viewed from the cosmos," Lecture Notes in Physics, vol. 737, pp. 949-974, 2008.

[29] J. M. Cline, "String cosmology," http://arxiv.org/abs/hep-th/ 0612129.

[30] C. P. Burgess, "Lectures on cosmic inflation and its potential stringy realizations," Classical and Quantum Gravity, vol. 24, no. 21, pp. S795-S852, 2007.

[31] L. McAllister and E. Silverstein, "String cosmology: a review," General Relativity and Gravitation, vol. 40, no. 2-3, pp. 565605, 2008.

[32] A. Mazumdar and J. Rocher, "Particle physics models of inflation and curvaton scenarios," http://arxiv.org/abs/1001.0993.

[33] E. Komatsu, "The pursuit of non-Gaussian fluctuations in the cosmic microwave background," http://arxiv.org/abs/ astro-ph/0206039.

[34] N. Bartolo, E. Komatsu, S. Matarrese, and A. Riotto, "NonGaussianity from inflation: theory and observations," Physics Reports, vol. 402, no. 3-4, pp. 103-266, 2004.

[35] M. Liguori, E. Sefusatti, J. R. Fergusson, and E. P. S. Shellard, "Primordial non-Gaussianity and bispectrum measurements in the cosmic microwave background and large-scale structure," http://arxiv.org/abs/1001.4707.

[36] L. Verde, "Non-Gaussianity from large-scale structure surveys," Advances in Astronomy, vol. 2010, Article ID 768675, 15 pages, 2010.

[37] N. Bartolo, S. Matarrese, and A. Riotto, "Non-Gaussianity of large-scale cosmic microwave background anisotropies beyond perturbation theory," Journal of Cosmology and Astroparticle Physics, no. 8, pp. 177-196, 2005.

[38] J. L. Lehners, "Ekpyrotic non-Gaussianity—a review," http:// arxiv.org/abs/1001.3125.

[39] R. K. Sachs and A. M. Wolfe, "Perturbations of a cosmological model and angular variations of themicrowave background," Astrophysical Journal, vol. 147, p. 73, 1967.

[40] J. Schwinger, "The special canonical group," Proceedings of the National Academy of Sciences of the United States of America, vol. 46, no. 10, pp. 1401-1415, 1960.

[41] J. Schwinger, "Brownian motion of a quantum oscillator," Journal of Mathematical Physics, vol. 2, no. 3, pp. 407-432, 1961.

[42] P. M. Bakshi and K. T. Mahanthappa, "Expectation value formalism in quantum field theory. I," Journal of Mathematical Physics, vol. 4, no. 1, pp. 1-11, 1963.

[43] L. V. Keldysh, "Diagram technique for nonequilibrium processes," JETP Letters, vol. 20, p. 1018, 1965.

[44] E. Calzetta and B. L. Hu, "Closed-time-path functional formalism in curved spacetime: application to cosmological back-reaction problems," Physical Review D, vol. 35, no. 2, pp. 495-509, 1987.

[45] S. Weinberg, "Quantum contributions to cosmological correlations," Physical Review D, vol. 72, no. 4, Article ID 043514, pp. 1-19, 2005.

[46] X. Chen and Y. Wang, "Quasi-single field inflation and nonGaussianities," Journal of Cosmology and Astroparticle Physics, vol. 2010, no. 4, article 027, 2010.

[47] J. Maldacena, "Non-gaussian features of primordial fluctuations in single field inflationary models," Journal of High Energy Physics, vol. 7, no. 5, pp. 233-264, 2003.

[48] V. Acquaviva, N. Bartolo, S. Matarrese, and A. Riotto, "Gauge-invariant second-order perturbations and nonGaussianity from inflation," Nuclear Physics B, vol. 667, no. 1-2, pp. 119-148, 2003. 
[49] D. Seery and J. E. Lidsey, "Primordial non-Gaussianities in single-field inflation," Journal of Cosmology and Astroparticle Physics, no. 6, pp. 45-70, 2005.

[50] X. Chen, M.-X. Huang, S. Kachru, and G. Shiu, "Observational signatures and non-Gaussianities of general singlefield inflation," Journal of Cosmology and Astroparticle Physics, no. 1, article 002, 2007.

[51] V. F. Mukhanov, H. A. Feldman, and R. H. Brandenberger, "Theory of cosmological perturbations," Physics Report, vol. 215, no. 5-6, pp. 203-333, 1992.

[52] E. J. Copeland, A. R. Liddle, D. H. Lyth, E. D. Stewart, and D. Wands, "False vacuum inflation with Einstein gravity," Physical Review D, vol. 49, no. 12, pp. 6410-6433, 1994.

[53] X. Chen, "Fine-tuning in DBI inflationary mechanism," Journal of Cosmology and Astroparticle Physics, vol. 2008, no. 12, 2008.

[54] E. Silverstein and D. Tong, "Scalar speed limits and cosmology: acceleration from D-cceleration," Physical Review D, vol. 70, no. 10, Article ID 103505, 18 pages, 2004.

[55] L. Randall and R. Sundrum, "Large mass hierarchy from a small extra dimension," Physical Review Letters, vol. 83, no. 17, pp. 3370-3373, 1999.

[56] S. B. Giddings, S. Kachru, and J. Polchinski, "Hierarchies from fluxes in string compactifications," Physical Review D, vol. 66, no. 10, Article ID 106006, 2002.

[57] X. Chen, S. Sarangi, S.-H. H. Tye, and J. Xu, "Is brane inflation eternal?" Journal of Cosmology and Astroparticle Physics, no. 11, article 015, 2006.

[58] D. Baumann and L. McAllister, "A microscopic limit on gravitational waves from D-brane inflation," Physical Review $D$, vol. 75, no. 12, Article ID 123508, 2007.

[59] D. H. Lyth, "What would we learn by detecting a gravitational wave signal in the cosmic microwavebackground anisotropy?" Physical Review Letters, vol. 78, no. 10, pp. 1861-1863, 1997.

[60] D. Babich, P. Creminelli, and M. Zaldarriaga, "The shape of non-Gaussianities," Journal of Cosmology and Astroparticle Physics, no. 8, pp. 199-217, 2004.

[61] X. Chen, "Running non-Gaussianities in Dirac-Born-Infeld inflation," Physical Review D, vol. 72, no. 12, Article ID 123518, 7 pages, 2005.

[62] C. T. Byrnes, S. Nurmi, G. Tasinato, and D. Wands, "Scale dependence of local $f_{\mathrm{NL}}$," Journal of Cosmology and Astroparticle Physics, vol. 2010, article 034, 2010.

[63] X. Chen, R. Easther, and E. A. Lim, "Large non-Gaussianities in single-field inflation," Journal of Cosmology and Astroparticle Physics, no. 6, article no. 023, 2007.

[64] X. Chen, R. Easther, and E. A. Lim, "Generation and characterization of large non-Gaussianities in single field inflation," Journal of Cosmology and Astroparticle Physics, vol. 2008, article 010, 2008.

[65] J. R. Fergusson and E. P. S. Shellard, "Shape of primordial non-Gaussianity and the CMB bispectrum," Physical Review $D$, vol. 80, no. 4, Article ID 043510, 26 pages, 2009.

[66] E. Komatsu and D. N. Spergel, "Acoustic signatures in the primary microwave background bispectrum," Physical Review D, vol. 63, no. 6, Article ID 063002, 2001.

[67] E. Komatsu, D. N. Spergel, and B. D. Wandelt, "Measuring primordial non-Gaussianity in the cosmic microwave background," Astrophysical Journal, vol. 634, no. 1, pp. 14-19, 2005.

[68] P. Creminelli, L. Senatore, and M. Zaldarriaga, "Estimators for local non-Gaussianities," Journal of Cosmology and Astroparticle Physics, no. 3, article no. 019, 2007.
[69] K. M. Smith and M. Zaldarriaga, "Algorithms for bispectra: forecasting, optimal analysis, and simulation," http:// arxiv.org/abs/astro-ph/0612571.

[70] A. P. S. Yadav, E. Komatsu, B. D. Wandelt, M. Liguori, F. K. Hansen, and S. Matarrese, "Fast estimator of primordial nonGaussianity from temperature and polarization anisotropies in the cosmic microwave background. II. Partial sky coverage and inhomogeneous noise," Astrophysical Journal, vol. 678, no. 2, pp. 578-582, 2008.

[71] J. R. Fergusson, M. Liguori, and E. P. S. Shellard, "General $\mathrm{CMB}$ and primordial bispectrum estimationi: mode expansion, map-making and measures of $f_{\mathrm{NL}}$, http://arxiv.org/ abs/0912.5516.

[72] J. Garriga and V. F. Mukhanov, "Perturbations in k-inflation," Physics Letters B, vol. 458, no. 2-3, pp. 219-225, 1999.

[73] M. Alishahiha, E. Silverstein, and D. Tong, "DBI in the sky: non-Gaussianity from inflation with a speed limit," Physical Review D, vol. 70, no. 12, Article ID 123505, 2004.

[74] A. Gruzinov, "Consistency relation for single scalar inflation," Physical Review D, vol. 71, no. 2, pp. 027301-2, 2005.

[75] C. Cheung, A. L. Fitzpatrick, J. Kaplan, L. Senatore, and P. Creminelli, "The effective field theory of inflation," Journal of High Energy Physics, vol. 2008, no. 3, article 014, 2008.

[76] N. Arkani-Hamed, P. Creminelli, S. Mukohyama, and M. Zaldarriaga, "Ghost inflation," Journal of Cosmology and Astroparticle Physics, no. 4, pp. 1-18, 2004.

[77] S. Weinberg, "Effective field theory for inflation," Physical Review D, vol. 77, no. 12, Article ID 123541, 2008.

[78] F. J. de Urries and J. Julve, "Ostrogradski formalism for higher-derivative scalar field theories," Journal of Physics A, vol. 31, no. 33, pp. 6949-6964, 1998.

[79] R. Woodard, "Avoiding dark energy with 1/R modifications of gravity," Lecture Notes in Physics, vol. 720, pp. 403-433, 2007.

[80] P. Creminelli, "On non-Gaussianities in single-field inflation," Journal of Cosmology and Astroparticle Physics, no. 10, pp. 53-62, 2003.

[81] P. Creminelli, A. Nicolis, L. Senatore, M. Tegmark, and M. Zaldarriaga, "Limits on non-Gaussianities from WMAP data," Journal of Cosmology and Astroparticle Physics, no. 5, article no. 004, 2006.

[82] B. Chen, Y. Wang, and W. Xue, "Inflationary non-Gaussianity from thermal fluctuations," Journal of Cosmology and Astroparticle Physics, vol. 2008, no. 5, article 014, 2008.

[83] D. Green, B. Horn, L. Senatore, and E. Silverstein, "Trapped inflation," Physical Review D, vol. 80, no. 6, Article ID 063533, 2009.

[84] I. G. Moss and C. Xiong, "Non-Gaussianity in fluctuations from warm inflation," Journal of Cosmology and Astroparticle Physics, no. 4, article no. 007, 2007.

[85] X. Chen, "Multithroat brane inflation," Physical Review D, vol. 71, no. 6, Article ID 063506, 5 pages, 2005.

[86] X. Chen, "Inflation from warped space," Journal of High Energy Physics, no. 8, pp. 1197-1231, 2005.

[87] S. E. Shandera and S.-H. H. Tye, "Observing brane inflation," Journal of Cosmology and Astroparticle Physics, no. 5, article no. 007, 2006.

[88] S. Kecskemeti, J. Maiden, G. Shiu, and B. Underwood, "DBI inflation in the tip region of a warped throat," Journal of High Energy Physics, vol. 2006, no. 9, article 076, 2006.

[89] G. Shiu and B. Underwood, "Observing the geometry of warped compactification via cosmic inflation," Physical Review Letters, vol. 98, no. 5, Article ID 051301, 2007. 
[90] S. Thomas and J. Ward, "IR inflation from multiple branes," Physical Review D, vol. 76, no. 2, Article ID 023509, 2007.

[91] R. Bean, S. E. Shandera, S.-H. Henry Tye, and J. Xu, "Comparing brane inflation to WMAP," Journal of Cosmology and Astroparticle Physics, no. 5, article no. 004, 2007.

[92] R. Bean, X. Chen, H. Peiris, and J. Xu, "Comparing infrared Dirac-Born-Infeld brane inflation to observations," Physical Review D, vol. 77, no. 2, Article ID 023527, 28 pages, 2008.

[93] C. Armendáriz-Picón, T. Damour, and V. Mukhanov, "kinflation," Physics Letters B, vol. 458, no. 2-3, pp. 209-218, 1999.

[94] M. Li, T. Wang, and Y. Wang, "General single field inflation with largepositive non-Gaussianity," Journal of Cosmology and Astroparticle Physics, vol. 2008, no. 3, article 028, 2008.

[95] A. J. Tolley and M. Wyman, "Equilateral non-Gaussianity from multifield dynamics," Physical Review D, vol. 81, no. 4, Article ID 043502, 5 pages, 2010.

[96] D. Langlois, S. Renaux-Petel, D. A. Steer, and T. Tanaka, "Primordial fluctuations and non-gaussianities in multifield Dirac-Born-Infeld inflation," Physical Review Letters, vol. 101, no. 6, Article ID 061301, 2008.

[97] D. Langlois, S. Renaux-Petel, D. A. Steer, and T. Tanaka, "Primordial perturbations and non-Gaussianities in DBI and general multi-field inflation," Physical Review D, vol. 78, Article ID 063523, 15 pages, 2008.

[98] F. Arroja, S. Mizuno, and K. Koyama, "Non-Gaussianity from the bispectrum in general multiple field inflation," Journal of Cosmology and Astroparticle Physics, vol. 2008, no. 8, article 015, 2008.

[99] D. Langlois, S. Renaux-Petel, and D. A. Steer, "Multi-field DBI inflation: introducing bulk forms and revisiting the gravitational wave constraints," Journal of Cosmology and Astroparticle Physics, vol. 2009, no. 4, article 021, 2009.

[100] S. Mizuno, F. Arroja, K. Koyama, and T. Tanaka, "Lorentz boost and non-Gaussianity in multifield DBI inflation," Physical Review D, vol. 80, no. 2, Article ID 023530, 10 pages, 2009.

[101] Y.-F. Cai and H.-Y. Xia, "Inflation with multiple sound speeds: a model of multiple DBI type actions and nonGaussianities," Physics Letters B, vol. 677, no. 5, pp. 226-234, 2009.

[102] X. Gao, "On cross-correlations between curvature and isocurvature perturbations during inflation," Journal of Cosmology and Astroparticle Physics, vol. 2010, no. 2, 2010.

[103] A. A. Starobinsky, "Spectrum of adiabatic perturbations in the Universe when there are singularities in the inflation potential," JETP Letters, vol. 55, p. 489, 1992.

[104] J. Adams, B. Cresswell, and R. Easther, "Inflationary perturbations from a potential with a step," Physical Review D, vol. 64, no. 12, Article ID 123514, 2001.

[105] J. Hamann, L. Covi, A. Melchiorri, and A. Slosar, "New constraints on oscillations in the primordial spectrum of inflationary perturbations," Physical Review D, vol. 76, no. 2, Article ID 023503, 14 pages, 2007.

[106] M. Joy, V. Sahni, and A. A. Starobinsky, "New universal local feature in the inflationary perturbation spectrum," Physical Review D, vol. 77, no. 2, Article ID 023514, 10 pages, 2008.

[107] R. Bean, X. Chen, G. Hailu, S.-H. Henry Tye, and J. Xu, "Duality cascade in brane inflation," Journal of Cosmology and Astroparticle Physics, vol. 2008, no. 3, article 026, 2008.

[108] L. A. Kofman, "Primordial perturbations from inflation," Physica Scripta, vol. T36, article 108, 1991.
[109] L. Wang and M. Kamionkowski, "Cosmic microwave background bispectrum and inflation," Physical Review D, vol. 61, no. 6, Article ID 063504, pp. 1-6, 2000.

[110] E. Komatsu, A. Kogut, M. R. Nolta et al., "First-year Wilkinson Microwave Anisotropy Probe (WMAP) observations: tests of Gaussianity," Astrophysical Journal, Supplement Series, vol. 148, no. 1, pp. 119-134, 2003.

[111] R. Flauger and E. Pajer, "Resonant Non-Gaussianity," http:// arxiv.org/abs/1002.0833.

[112] S. Hannestad, T. Haugbølle, P. R. Jarnhus, and M. S. Sloth, "Non-gaussianity from axion monodromy inflation," Journal of Cosmology and Astroparticle Physics, vol. 2010, no. 6, 2010.

[113] S. Hannestad, T. Haugbølle, P. R. Jarnhus, and M. S. Sloth, "Non-gaussianity from axion monodromy inflation," Journal of Cosmology and Astroparticle Physics, vol. 2010, no. 6, 2010.

[114] G. Hailu and S.-H. Henry Tye, "Structures in the gauge/gravity duality cascade," Journal of High Energy Physics, vol. 2007, no. 8, 2007.

[115] E. Silverstein and A. Westphal, "Monodromy in the CMB: gravity waves and string inflation," Physical Review D, vol. 78, no. 10, Article ID 106003, 21 pages, 2008.

[116] L. McAllister, E. Silverstein, and A. Westphal, "Gravity waves and linear inflation from Axion Monodromy," http://arxiv .org/abs/0808.0706.

[117] J. Martin, "Trans-planckian problem of inflationary cosmology," Physical Review D, vol. 63, no. 12, Article ID 123501, 16 pages, 2001.

[118] U. H. Danielsson, "Note on inflation and trans-Planckian physics," Physical Review D, vol. 66, no. 2, Article ID 023511, 5 pages, 2002.

[119] R. H. Brandenberger, "Theory of cosmological perturbations and applications to superstring cosmology," in Proceedings of the NATO Advanced Study Institute on String Theory: From Gauge Interactions to Cosmology Cargèse, pp. 79-116, June 2004.

[120] B. Greene, K. Schalm, J. P. van der Schaar, and G. Shiu, "Extracting new physics from the CMB," in Proceedings of 22nd Texas Symposium on Relativistic Astrophysics, Stanford, Calif, USA, December 2004.

[121] P. D. Meerburg, J. P. Van Der Schaar, and M. G. Jackson, "Bispectrum signatures of a modified vacuum in single field inflation with a small speed of sound," Journal of Cosmology and Astroparticle Physics, vol. 2010, no. 2, 2010.

[122] R. Holman and A. J. Tolley, "Enhanced non-Gaussianity from excited initial states," Journal of Cosmology and Astroparticle Physics, vol. 2008, no. 5, article 001, 2008.

[123] P. D. Meerburg, J. P. van der Schaar, and P. S. Corasaniti, "Signatures of initial state modifications on bispectrum statistics," Journal of Cosmology and Astroparticle Physics, vol. 2009, no. 5, article 018, 2009.

[124] J. Martin, A. Riazuelo, and M. Sakellariadou, "Nonvacuum initial states for cosmological perturbations of quantummechanical origin," Physical Review D, vol. 61, no. 8, Article ID 083518, 15 pages, 2000.

[125] A. Gangui, J. Martin, and M. Sakellariadou, "Single field inflation and non-Gaussianity," Physical Review D, vol. 66, no. 8, Article ID 083502, 3 pages, 2002.

[126] X. Chen and Y. Wang, "Large non-Gaussianities with intermediate shapes from quasi-single-field inflation," Physical Review D, vol. 81, no. 6, Article ID 063511, 5 pages, 2010.

[127] D. S. Salopek and J. R. Bond, "Nonlinear evolution of long-wavelength metric fluctuations in inflationary models," Physical Review D, vol. 42, no. 12, pp. 3936-3962, 1990. 
[128] N. Bartolo, S. Matarrese, and A. Riotto, "Non-Gaussianity from inflation," Physical Review D, vol. 65, no. 10, Article ID 103505, 2002.

[129] F. Bernardeau and J.-P. Uzan, "Non-Gaussianity in multifield inflation," Physical Review D, vol. 66, no. 10, 14 pages, 2002.

[130] F. Bernardeau and J.-P. Uzan, "Inflationary models inducing non-Gaussian metric fluctuations," Physical Review D, vol. 67, no. 12, Article ID 121301, 5 pages, 2003.

[131] G. I. Rigopoulos, E. P. S. Shellard, and B. J. W. Van Tent, "Nonlinear perturbations in multiple-field inflation," Physical Review D, vol. 73, no. 8, Article ID 083521, pp. 111, 2006.

[132] G. I. Rigopoulos, E. P. S. Shellard, and B. J. W. van Tent, "Large non-Gaussianity in multiple-field inflation," Physical Review D, vol. 73, no. 8, Article ID 083522, pp. 1-19, 2006.

[133] D. Seery and J. E. Lidsey, "Primordial non-Gaussianities from multiple-field inflation," Journal of Cosmology and Astroparticle Physics, no. 9, pp. 187-210, 2005.

[134] F. Vernizzi and D. Wands, "Non-Gaussianities in two-field inflation," Journal of Cosmology and Astroparticle Physics, vol. 2006, no. 5, article 019, 2006.

[135] D. Mulryne, D. Seery, and D. Wesley, "Non-Gaussianity constrains hybrid inflation," http://arxiv.org/abs/0911.3550.

[136] J. O. Gong, C. Lin, and Y. Wang, "Non-Gaussianity from false vacuum inflation: old curvaton scenario," Journal of Cosmology and Astroparticle Physics, vol. 2010, no. 3, article 004, 2010.

[137] A. Gangui, F. Lucchin, S. Matarrese, and S. Mollerach, "The three-point correlation function of the cosmic microwave background in inflationary models," Astrophysical Journal, vol. 430, no. 2, pp. 447-457, 1994.

[138] L. Verde, L. Wang, A. F. Heavens, and M. Kamionkowski, "Large-scale structure, the cosmic microwave background and primordial non-Gaussianity," Monthly Notices of the Royal Astronomical Society, vol. 313, no. 1, pp. 141-147, 2000.

[139] A. A. Starobinsky, "Multicomponent de Sitter (Inflationary) stages and the generation of perturbations," JETP Letters, vol. 42, p. 152, 1985.

[140] M. Sasaki and E. D. Stewart, "A general analytic formula for the spectral index of the density perturbations produced during inflation," Progress of Theoretical Physics, vol. 95, no. 1, pp. 71-78, 1996.

[141] D. H. Lyth and Y. Rodríguez, "Inflationary prediction for primordial non-gaussianity," Physical Review Letters, vol. 95, no. 12, Article ID 121302, 4 pages, 2005.

[142] A. Linde and V. Mukhanov, "Non-Gaussian isocurvature perturbations from inflation," Physical Review D, vol. 56, no. 2, pp. R535-R539, 1997.

[143] D. H. Lyth and D. Wands, "Generating the curvature perturbation without an inflaton," Physics Letters B, vol. 524, no. 1-2, pp. 5-14, 2002.

[144] T. Moroi and T. Takahashi, "Effects of cosmological moduli fields on cosmic microwave background," Physics Letters B, vol. 522, no. 3-4, pp. 215-221, 2001.

[145] T. Moroi and T. Takahashi, "Erratum: effects of cosmological moduli fields on cosmic microwave background (Physics Letters, Section B: Nuclear, Elementary Particle (2001) 522 (215) PII: S0370269301012953)," Physics Letters B, vol. 539, no. 3-4, p. 303, 2002.

[146] D. H. Lyth, C. Ungarelli, and D. Wands, "Primordial density perturbation in the curvaton scenario," Physical Review D, vol. 67, no. 2, Article ID 023503, 2003.
[147] N. Bartolo, S. Matarrese, and A. Riotto, "Non-Gaussianity in the curvaton scenario," Physical Review D, vol. 69, no. 4, Article ID 043503, 2004.

[148] Q.-G. Huang, "Large non-Gaussianity implication for curvaton scenario," Physics Letters B, vol. 669, no. 5, pp. 260-265, 2008.

[149] M. Li, C. Lin, T. Wang, and Y. Wang, "Non-Gaussianity, isocurvature perturbation, gravitational waves, and a no-go theorem for the isocurvaton," Physical Review D, vol. 79, no. 6, Article ID 063526, 10 pages, 2009.

[150] K. Enqvist and T. Takahashi, "Signatures of non-Gaussianity in the curvaton model," Journal of Cosmology and Astroparticle Physics, vol. 2008, no. 9, article 012, 2008.

[151] Q.-G. Huang and Y. Wang, "Curvaton dynamics and the non-linearity parameters in the curvaton model," Journal of Cosmology and Astroparticle Physics, vol. 2008, no. 9, article 025, 2008.

[152] E. Komatsu, J. Dunkley, M. R. Nolta et al., "Five-year wilkinson microwave anisotropy probe observations: cosmological interpretation," Astrophysical Journal, Supplement Series, vol. 180, no. 2, pp. 330-376, 2009.

[153] C. Gordon, D. Wands, B. A. Bassett, and R. Maartens, "Adiabatic and entropy perturbations from inflation," Physical Review D, vol. 63, no. 2, Article ID 023506, 11 pages, 2001.

[154] G. Dvali, A. Gruzinov, and M. Zaldarriaga, "New mechanism for generating density perturbations from inflation," Physical Review D, vol. 69, no. 2, Article ID 023505, 6 pages, 2004.

[155] L. Kofman, "Probing string theory with modulated cosmological fluctuations," http://arxiv.org/abs/astro-ph/0303614.

[156] K. Enqvist, A. Jokinen, A. Mazumdar, T. Multamäki, and A. Väihkönen, "Non-gaussianity from preheating," Physical Review Letters, vol. 94, no. 16, Article ID 161301, 4 pages, 2005.

[157] A. Jokinen and A. Mazumdar, "Very large primordial nonGaussianity from multiple fields: application to massless preheating," Journal of Cosmology and Astroparticle Physics, no. 4, pp. 25-45, 2006.

[158] N. Barnaby and J. M. Cline, "Non-Gaussianity from tachyonic preheating in hybrid inflation," Physical Review D, vol. 75, no. 8, Article ID 086004, 14 pages, 2007.

[159] A. Chambers and A. Rajantie, "Lattice calculation of nongaussian density perturbations from the massless preheating inflationary model," Physical Review Letters, vol. 100, no. 4, Article ID 041302, 4 pages, 2008.

[160] A. Chambers and A. Rajantie, "Erratum: lattice calculation of non-Gaussian density perturbations from the massless preheating inflationary model (Physical Review Letters (2008) 100 (041302))," Physical Review Letters, vol. 101, no. 14, Article ID 149903, 1 pages, 2008.

[161] J. R. Bond, A. V. Frolov, Z. Huang, and L. Kofman, "Nongaussian curvature spikes from chaotic billiards in inflation preheating," Physical Review Letters, vol. 103, no. 7, Article ID 071301, 4 pages, 2009.

[162] N. Bartolo, E. Dimastrogiovanni, S. Matarrese, and A. Riotto, "Anisotropic bispectrum of curvature perturbations from primordial non-abelian vector fields," Journal of Cosmology and Astroparticle Physics, vol. 2009, no. 10, article 015, 2009.

[163] C.A. Valenzuela-Toledo, Y. Rodríguez, and D. H. Lyth, "NonGaussianity at tree and one-loop levels from vector field perturbations," Physical Review D, vol. 80, no. 10, Article ID 103519, 7 pages, 2009. 
[164] E. Dimastrogiovanni, N. Bartolo, S. Matarrese, and A. Riotto, "Non-Gaussianity and statisticalanisotropy from vector field populated inflationary models," http://arxiv.org/ abs/1001.4049.

[165] N. Barnaby and J. M. Cline, "Large non-Gaussianity from non-local inflation," Journal of Cosmology and Astroparticle Physics, vol. 2007, no. 7, article 017, 2007.

[166] N. Barnaby and J. M. Cline, "Predictions for non-Gaussianity from non-local inflation," Journal of Cosmology and Astroparticle Physics, vol. 2008, no. 6, article 030, 2008.

[167] E. I. Buchbinder, J. Khoury, and B. A. Ovrut, "NonGaussianities in new ekpyrotic cosmology," Physical Review Letters, vol. 100, no. 17, Article ID 171302, 4 pages, 2008.

[168] K. Koyama, S. Mizuno, F. Vernizzi, and D. Wands, "NonGaussianities from ekpyrotic collapse with multiple fields," Journal of Cosmology and Astroparticle Physics, no. 11, pp. 121, 2007.

[169] J. Khoury and F. Piazza, "Rapidly-varying speed of sound, scale invariance and non-Gaussian signatures," Journal of Cosmology and Astroparticle Physics, vol. 2009, no. 7, article 026, 2009.

[170] J.-L. Lehners and S. Renaux-Petel, "Multifield cosmological perturbations at third order and the ekpyrotic trispectrum," Physical Review D, vol. 80, no. 6, Article ID 063503, 18 pages, 2009.

[171] J.-L. Lehners and P. J. Steinhardt, "Non-Gaussianity generated by the entropic mechanism in bouncing cosmologies made simple," Physical Review D, vol. 80, no. 10, Article ID 103520, 5 pages, 2009.

[172] A. Slosar, C. Hirata, U. Seljak, S. Ho, and N. Padmanabhan, "Constraints on local primordial non-Gaussianity from large scale structure," Journal of Cosmology and Astroparticle Physics, vol. 2008, no. 8, 2008.

[173] A. P.S. Yadav and B. D. Wandelt, "Evidence of primordial non-gaussianity $\left(f_{N L}\right)$ in the wilkinson microwave anisotropy probe 3-year data at 2.8 $\sigma$," Physical Review Letters, vol. 100, no. 18, Article ID 181301, 4 pages, 2008.

[174] K. M. Smith, L. Senatore, and M. Zaldarriaga, "Optimal limits on $f_{N L}^{\text {local }}$ from WMAP 5-year data," Journal of Cosmology and Astroparticle Physics, vol. 2009, no. 9, 2009.

[175] L. Senatore, K. M. Smith, and M. Zaldarriaga, "NonGaussianities in single field inflation and their optimal limits from the WMAP 5-year data," Journal of Cosmology and Astroparticle Physics, vol. 2010, no. 1, 2010.

[176] N. Dalal, O. Doré, D. Huterer, and A. Shirokov, "Imprints of primordial non-Gaussianities on large-scale structure: scaledependent bias and abundance of virialized objects," Physical Review D, vol. 77, no. 12, Article ID 123514, 17 pages, 2008.

[177] V. Desjacques and U. Seljak, "Signature of primordial nonGaussianity of $\varphi^{3}$ type in the mass function and bias of dark matter haloes," Physical Review D, vol. 81, no. 2, 2010.

[178] L. Verde and S. Matarrese, "Detectability of the effect of inflationary non-gaussianity on halo bias," Astrophysical Journal, vol. 706, no. 1, part 2, pp. L91-L95, 2009.

[179] M. Roncarelli, L. Moscardini, E. Branchini et al., "Imprints of primordial non-Gaussianities in X-ray and SZ signals from galaxy clusters," Monthly Notices of the Royal Astronomical Society, vol. 402, no. 2, pp. 923-933, 2010.

[180] $\varnothing$. Rudjord, F. K. Hansen, X. Lan, M. Liguori, D. Marinucci, and S. Matarrese, "An estimate of the primordial nongaussianity parameter $f_{\mathrm{NL}}$ using the needlet bispectrum from wmap," Astrophysical Journal, vol. 701, no. 1, pp. 369-376, 2009.
[181] $\varnothing$. Rudjord, F. K. Hansen, X. Lan, M. Liguori, D. Marinucci, and S. Matarrese, "Directional variations of the nongaussianity parameter $f_{\mathrm{NL}}$," Astrophysical Journal, vol. 708, no. 2, pp. 1321-1325, 2010.

[182] J. Smidt, A. Amblard, P. Serra, and A. Cooray, "Measurement of primordial non-Gaussianity using the WMAP 5-year temperature skewness power spectrum," Physical Review D, vol. 80, no. 12, 19 pages, 2009.

[183] P. Cabella, et al., "Foreground influence on primordial non-Gaussianity estimates: needlet analysis ofWMAP 5-year data," http://arxiv.org/abs/0910.4362.

[184] M. Bucher, B. Van Tent, and C. S. Carvalho, "Detecting bispectral acoustic oscillations from inflationusing a new flexible estimator," http://arxiv.org/abs/0911.1642.

[185] M. LoVerde, A. Miller, S. Shandera, and L. Verde, "Effects of scale-dependent non-Gaussianity on cosmological structures," Journal of Cosmology and Astroparticle Physics, vol. 4, article 014, 2008.

[186] E. Sefusatti, M. Liguori, A. P.S. Yadav, M. G. Jackson, and E. Pajer, "Constraining running non-gaussianity," Journal of Cosmology and Astroparticle Physics, vol. 2009, no. 12, 2009.

[187] X. Chen, B. Hu, M.-X. Huang, G. Shiu, and Y. Wang, "Large primordial trispectra in general single field inflation," Journal of Cosmology and Astroparticle Physics, vol. 2009, no. 8, 2009.

[188] F. Arroja, S. Mizuno, K. Koyama, and T. Tanaka, "Full trispectrum in single field DBI inflation," Physical Review D, vol. 80, no. 4, Article ID 043527, 14 pages, 2009.

[189] M.-X. Huang and G. Shiu, "Inflationary trispectrum for models with large non-Gaussianities," Physical Review D, vol. 74, no. 12, 5 pages, 2006.

[190] F. Arroja and K. Koyama, "Non-Gaussianity from the trispectrum in general single field inflation," Physical Review D, vol. 77, no. 8, Article ID 083517, 14 pages, 2008.

[191] X. Gao and B. Hu, "Primordial trispectrum from entropy perturbations in multifield DBI model," Journal of Cosmology and Astroparticle Physics, vol. 2009, no. 8, 2009.

[192] G. Xian, L. Miao, and L. Chunshan, "Primordial nonGaussianities from the trispectra in multiple field inflationary models," Journal of Cosmology and Astroparticle Physics, vol. 2009, no. 11, 2009.

[193] S. Mizuno, F. Arroja, and K. Koyama, "Full quantum trispectrum in multifield DBI inflation," Physical Review D, vol. 80, no. 8, Article ID 083517, 18 pages, 2009.

[194] D. Seery, J. E. Lidsey, and M. S. Sloth, "The inflationary trispectrum," Journal of Cosmology and Astroparticle Physics, vol. 2007, no. 1, 2007.

[195] D. Seery and J. E. Lidsey, "Non-Gaussianity from the inflationary trispectrum," Journal of Cosmology and Astroparticle Physics, vol. 2007, no. 1, 2007.

[196] D. Seery, M. S. Sloth, and F. Vernizzi, "Inflationary trispectrum from graviton exchange," Journal of Cosmology and Astroparticle Physics, vol. 2009, no. 3, 2009.

[197] P. Adshead, R. Easther, and E. A. Lim, “" in-in” formalism and cosmological perturbations," Physical Review D, vol. 80, no. 8, 2009.

[198] N. Bartolo, E. Dimastrogiovanni, S. Matarrese, and A. Riotto, "Anisotropic trispectrum of curvature perturbations induced by primordial non-Abelian vector fields," Journal of Cosmology and Astroparticle Physics, vol. 11, article 028, 2009.

[199] C.A. Valenzuela-Toledo and Y. Rodríguez, "Non-gaussianity from the trispectrum and vector field perturbations," Physics Letters B, vol. 685, no. 2-3, pp. 120-127, 2010. 
[200] P. Creminelli and M. Zaldarriaga, "A single-field consistency relation for the three-point function," Journal of Cosmology and Astroparticle Physics, no. 10, pp. 101-107, 2004.

[201] C. Cheung, A. L. Fitzpatrick, J. Kaplan, and L. Senatore, "On the consistency relation of the three-point function in single-field inflation," Journal of Cosmology and Astroparticle Physics, vol. 2008, no. 2, 2008.

[202] S. Renaux-Petel, "Combined local and equilateral nonGaussianities from multifield DBI inflation," Journal of Cosmology and Astroparticle Physics, vol. 2009, no. 10, 2009. 

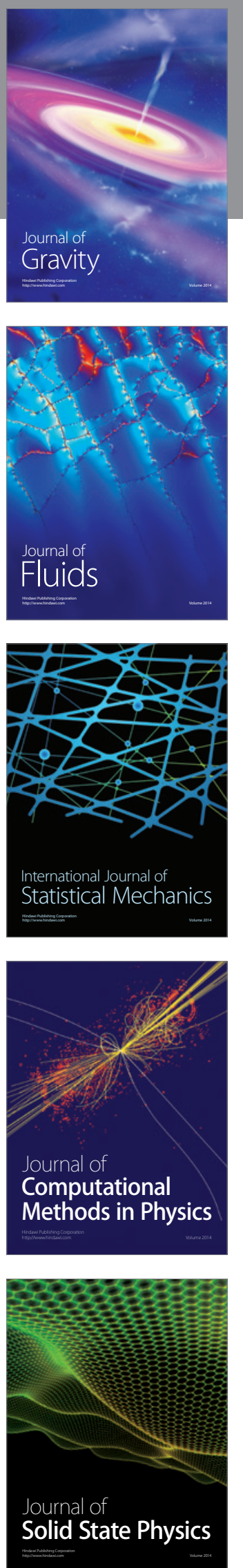

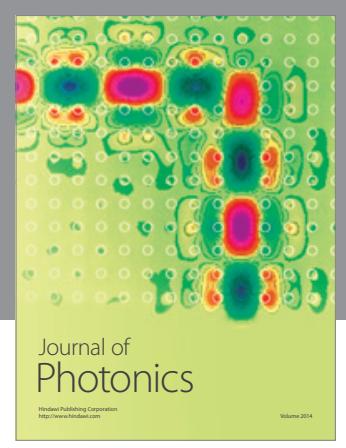

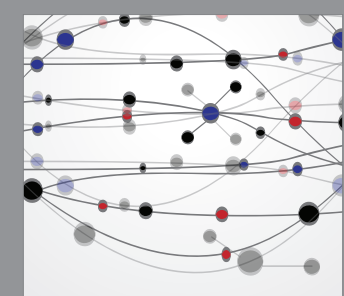

The Scientific World Journal
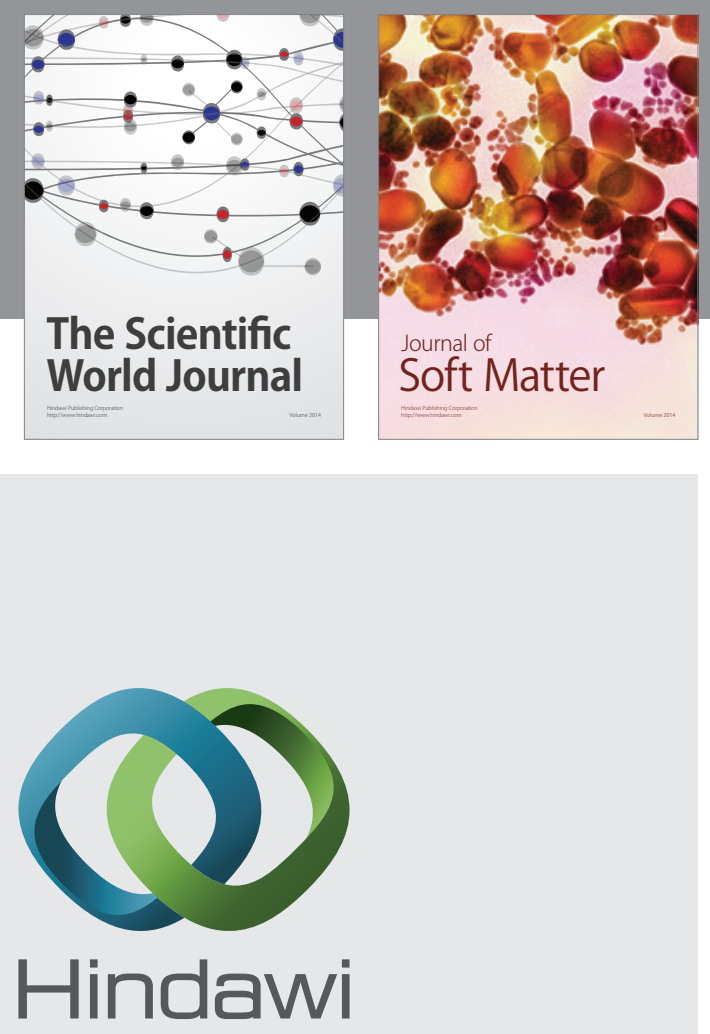

Submit your manuscripts at

http://www.hindawi.com
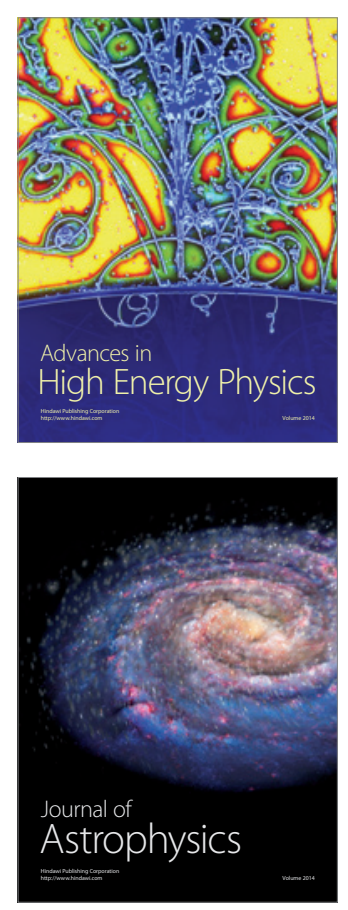
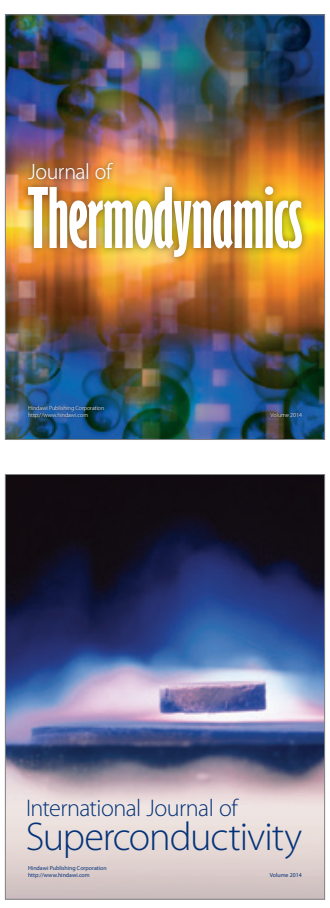
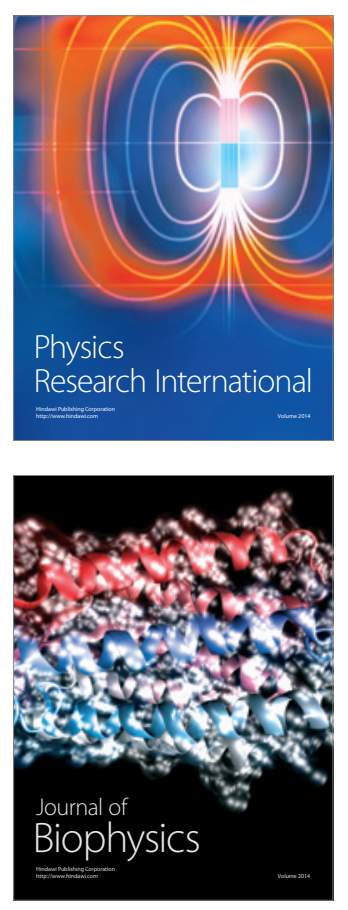
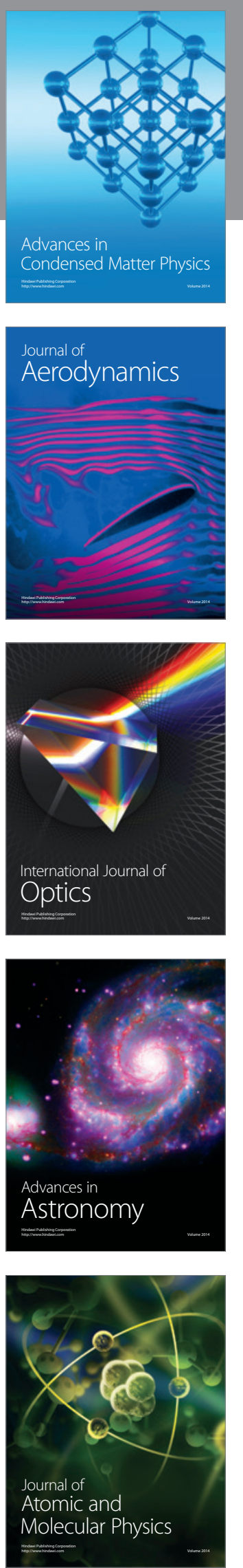